\title{
Advances in Research on Chemical Constituents and Their Biological Activities of the Genus Actinidia
}

\author{
Jin-Tao Ma ${ }^{1} \cdot$ Da-Wei Li ${ }^{2} \cdot$ Ji-Kai Liu $^{1} \cdot$ Juan He ${ }^{1}$
}

Received: 26 July 2021 / Accepted: 15 September 2021 / Published online: 30 September 2021

(c) The Author(s) 2021

\begin{abstract}
Kiwi, a fruit from plants of the genus Actinidia, is one of the famous fruits with thousand years of edible history. In the past twenty years, a great deal of research has been done on the chemical constituents of the Actinidia species. A large number of secondary metabolites including triterpenoids, flavonoids, phenols, etc. have been identified from differents parts of Actinidia plants, which exhibited significant in vitro and in vivo pharmacological activities including anticancer, anti-inflammatory, neuroprotective, anti-oxidative, anti-bacterial, and anti-diabetic activities. In order to fully understand the chemical components and biological activities of Actinidia plants, and to improve their further research, development and utilization, this review summarizes the compounds extracted from different parts of Actinidia plants since 1959 to 2020, classifies the types of constituents, reports on the pharmacological activities of relative compounds and medicinal potentials.
\end{abstract}

Keywords Actinidia chemical constituents $\cdot$ Isolation $\cdot$ Biological activities

\section{Introduction}

With the development of natural product research, a huge number of chemical constituents have been identified from natural resources. There is no doubt that the research on the chemical composition of fruits, including trace elements, has greatly improved the application prospects of these fruits. With no exception, it is the same to kiwifruit, one of the most prestigious fruits with a long history of eating $[1,2]$. Kiwi belongs to plants of the genus Actinidia comprising more than 70 species around the world [3]. Some of these plants are proven to have a wide range of medicinal activities. For example, A. valvata, whose root is known as "Mao-Ren-Shen" in traditional Chinese medicine, exhibits antitumor and anti-inflammatory activities and has been used for the treatment of hepatoma, lung carcinoma

Juan He

2015048@mail.scuec.edu.cn

1 School of Pharmaceutical Sciences, National Demonstration Center for Experimental Ethnopharmacology Education, South-Central University for Nationalities, Wuhan 430074, People's Republic of China

2 Center of Economic Botany, Core Botanical Gardens, Chinese Academy of Sciences, Wuhan 430074,

People's Republic of China and myeloma for a long time $[4,5]$. The roots of A. chinensis Planch, called "Teng-Li-Gen" usually, were used as a traditional Chinese medicine for the treatment of various cancers, such as esophagus cancer, liver cancer, and gastric cancer [6]. In the past two decades, great research had been accomplished about exploring the chemical composition of Actinidia plants. These studies have greatly promoted the understanding of the chemical components and functions of the Actinidia plant. According to literature survey, 12 Actinidia species including A. valvata, A. chinensis, A. argute, A. polygama, A. kolomikta, A. eriantha, A. macrosperma, A. deliciosa, A. chrysantha, A. rufa, A. indochinensis, and A. valvata were reported for their natural products. This review systematically summarizes the chemical components and their biological activities from different parts of 12 Actinidia species from 1959 to 2020. According to structure types, a total of 325 molecules have been collected including terpeniods, phenols, and other small groups (Fig. 1). Names and isolation information were listed in the tables, while the biological activities of the extracts or compounds were discussed in the text. 


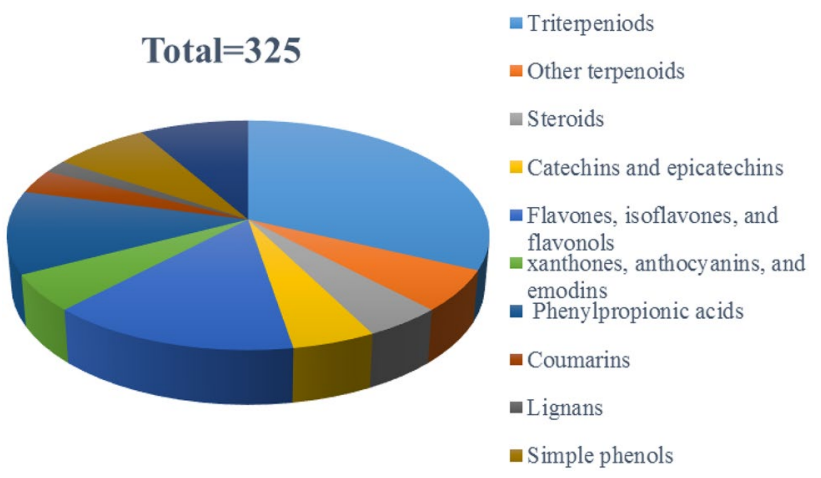

Fig. 1 Constituents proportion of 12 Actinidia plants

\section{Chemical Constituents}

\subsection{Terpenoids}

In recent years, a large number of terpenoids were isolated from many Actinidia species. Among them, triterpenes account for the vast majority that are mainly composed by several normal frameworks including ursane-type, oleanane-type, and lupane-type. Of the total 325 compounds in this review, 104 are triterpenoids. From the literature review, ursolic acids and their saponins are undoubtedly the most abundant in Actinidia species.

\subsubsection{Ursane Triterpenoids}

Ursane-type triterpenes are characterized of ursolic acid and its saponins, possessing a 6/6/6/6/6-fused carbon skeleton. A total of 76 ursane-type triterpenoids (1-76) have been identified from plants of the genus Actinidia (Fig. 2, Table 1). Ursolic acid ( $3 \beta$-Hydroxyurs-12-en-28-oic acid 1) [7], is one of the most frequently obtained compound in many kinds of kiwifruit plants with unique flavor. Great attention had been paid on biological activities about ursolic acid, attracting much interest in recent years. Ursolic acid exhibits different pharmacological activities, including anti-cancer, amylolytic enzyme inhibitors, cytotoxicity, downregulating thymic stromal lymphopoietin and others [7-11].

Compounds 2-7 are ursane triterpenoids featuring with two hydroxyl groups. Compound $\mathbf{3}$ ( $2 \alpha$-Hydroxyursolic acid) was tested for its antiproliferative activity and cytotoxicity in MDA-MB-231 human breast cancer cells through the methylene blue assay. It significantly down-regulated expressions of TRAF2, PCNA, cyclin D1, and CDK4 and up-regulated the expressions of p-ASK1, p-p38, p-p53, and p-21. Furthermore, it induced apoptosis in MDA-MB-231 cell by significantly increasing the Bax/ Bcl-2 ratio and inducing the cleaved caspase-3 [12]. Compound 4 exhibited inhibitory activity on pancreatic lipase with an $\mathrm{IC}_{50}$ value of $20.42 \pm 0.95 \mu \mathrm{M}$ [13]. It also showed cytotoxicity to human lung adenocarcinoma (A549), ovarian cancer (SK-OV-3), skin melanoma (SK-MEL-2), and colon cancer (HCT-15) cell lines with $\mathrm{IC}_{50}$ values ranging from 11.96 to $14.11 \mu \mathrm{M}$ [14-17].

Compounds 7-14 are trihydroxy-ursolic acid derivatives. In early 1992, Sashida et al. reported the isolation of 8-10. Compounds 8 and $\mathbf{9}$ were evaluated for their cytotoxicity against A549 cells, LOVO cells, and HepG2 cells with $\mathrm{IC}_{50}$ values of $32.9,31.6,35.7 \mu \mathrm{g} / \mathrm{mL}$ respectively for $\mathbf{8}$ and 34.6 , $13.9,34.5 \mu \mathrm{g} / \mathrm{mL}$ respectively for $9[18,19]$. Compounds 17-26 are ursolic acids with four or five hydroxy groups. $\mathrm{Xu}$ et al. reported $\mathbf{1 7}$ from roots of $A$. valvata, this compound exhibited weak cytotoxicity against A549, LOVO and HepG2 cell lines with $\mathrm{IC}_{50}$ values of above $100 \mu \mathrm{g} / \mathrm{mL}$ [20]. $2 \alpha, 3 \alpha, 19 \alpha, 24$-Tetrahydroxyurs-12-en-28-oic acid $\mathbf{2 0}$ was separated from the leaves of $A$. valvata which showed cytotoxicity against PLC, Hep3B, HepG2, HeLa, SW480, MCF-7 and Bel7402 in vitro [21]. A new polyoxygenated triterpenoid $(2 \beta, 3 \alpha, 6 \alpha)-2,3,6,20,23,30$-hexahydroxyurs-12en-28-oic acid 26 was obtained from the roots of $A$. valvata DUNN, it exhibited moderate cytotoxic activity against BEL-7402 and SMMC-7721 tumor cell lines in vitro [22].

Compound 30 ( $3 \beta$-O-acetylursolic acid) was isolated from the fruit galls of $A$. polygama and the structure was elucidated on the basis of chemical and spectral evidence. It was reported to be a mixed-type protein tyrosine phosphatase 1B (PTP1B) inhibitor with an $\mathrm{IC}_{50}$ value of $4.8 \pm 0.5 \mu \mathrm{M}[23,24]$. Isolation of the antiviral active ingredient of A. chinensis root bark gave fupenzic acid 40, which showed moderate inactivity under the concentration of $100 \mu \mathrm{g} / \mathrm{mL}$ [25]. Callus tissue from the stems of $A$. arguta (Actinidiaceae) produced three ursane-type triterpenes including ursolaldehyde 41, $\alpha$-amyrin $\mathbf{4 2}$, and uvaol $\mathbf{4 3}$ [26]. Of them, compound $\mathbf{4 3}$ showed anti-inflammatory, anticancer, and wound healing activities [27-29]. Antiinflammatory properties of $\mathbf{4 3}$ on DSS-induced colitis and LPS-stimulated macrophages have been explored detailly and completely. It showed excellent potential of NO production inhibition. It could attenuate disease activity index (DAI), colon shortening, colon injury, and colonic myeloperoxidase activity in DSS-induced colitis mice. What's more, studies on LPS challenged murine macrophage 

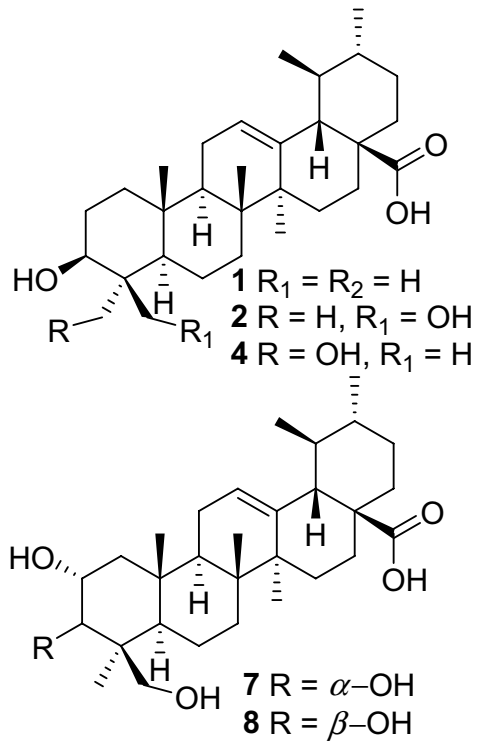

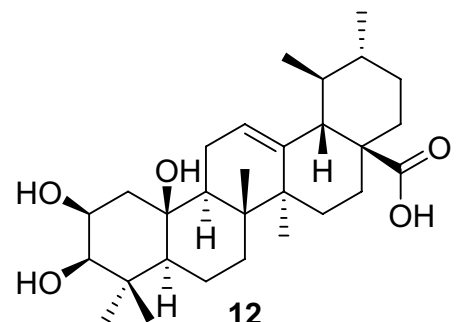

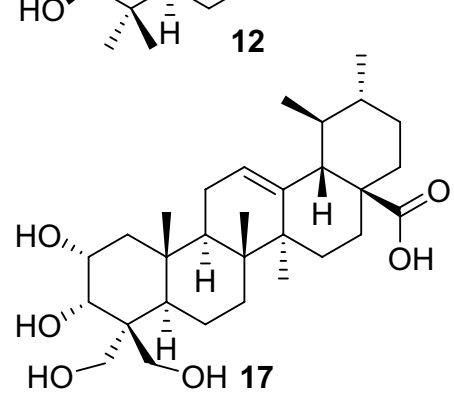

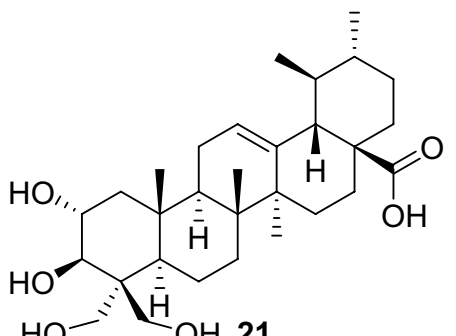

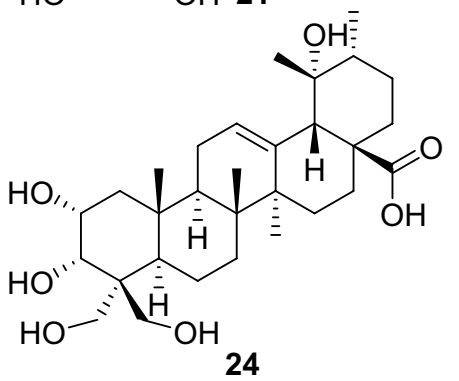

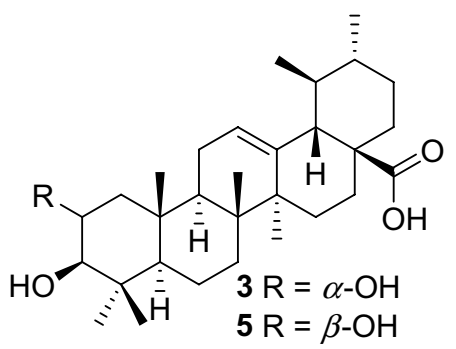

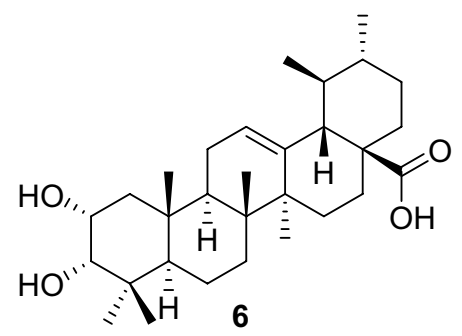<smiles>[R]O[R]([R])(O)C(=O)O</smiles>

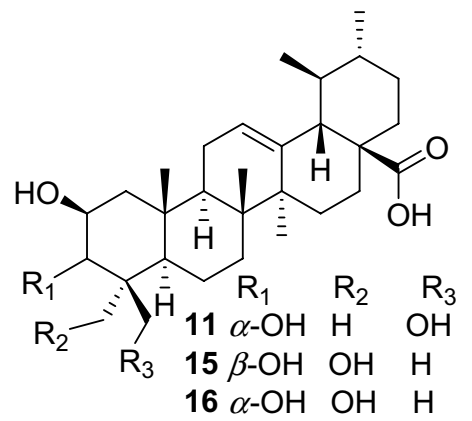

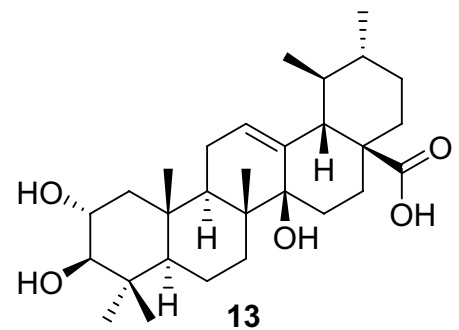

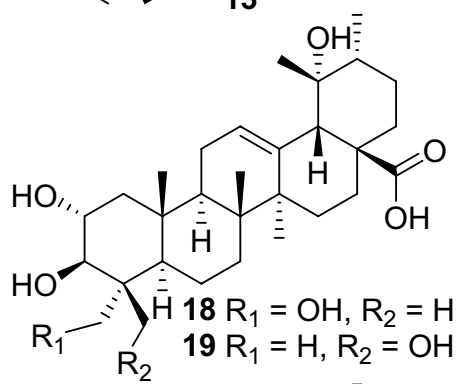

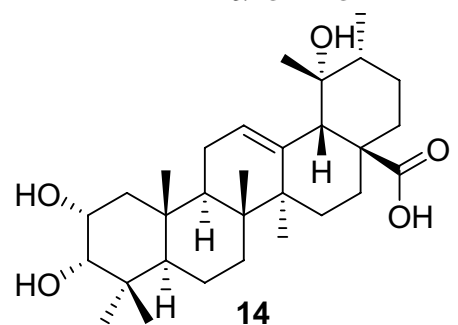

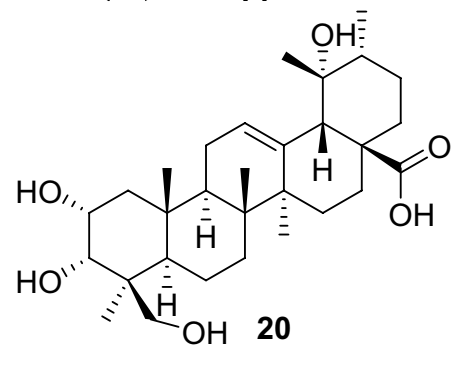

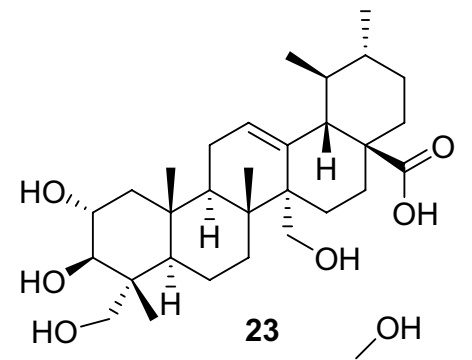

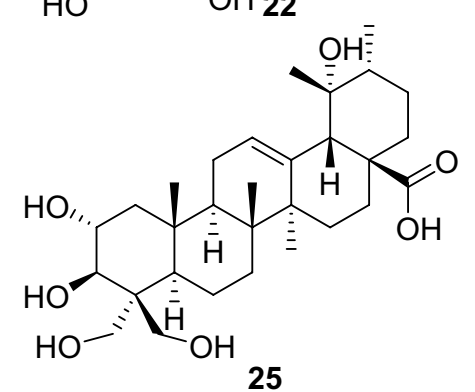

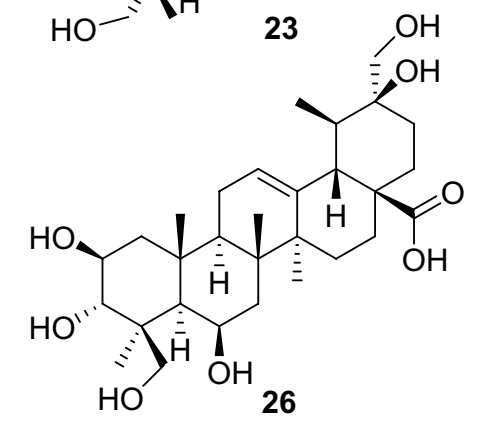

Fig. 2 Structures of ursane triterpenoids 1-76 from Actinidia plants 


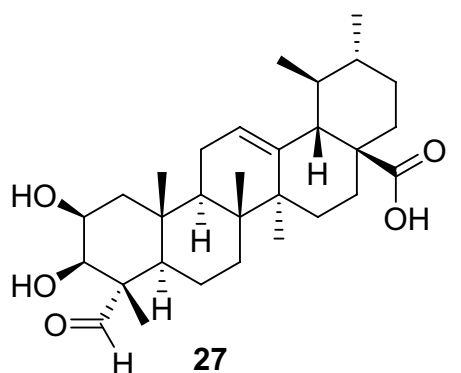

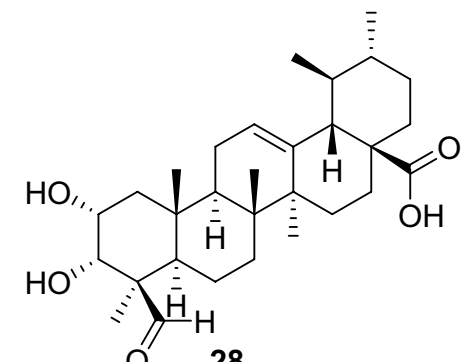

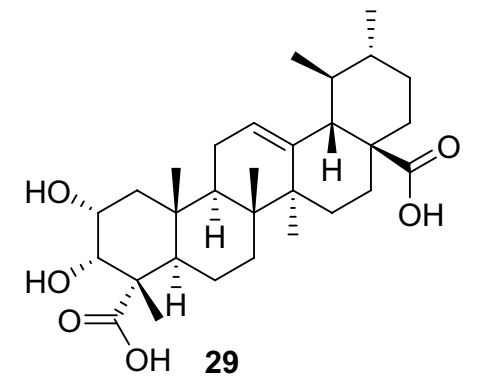

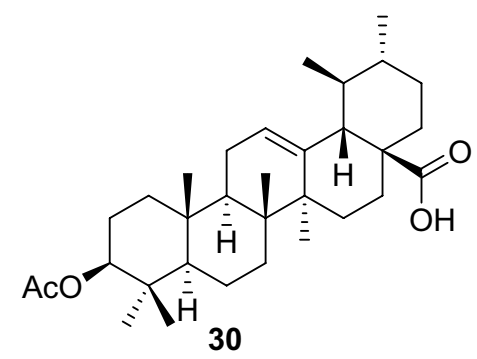

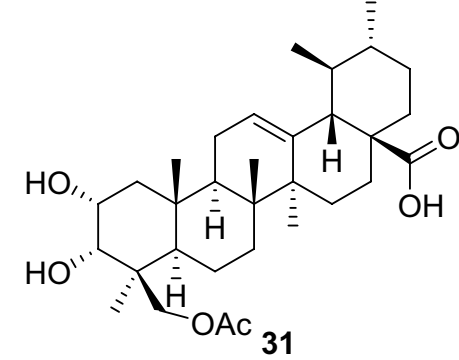

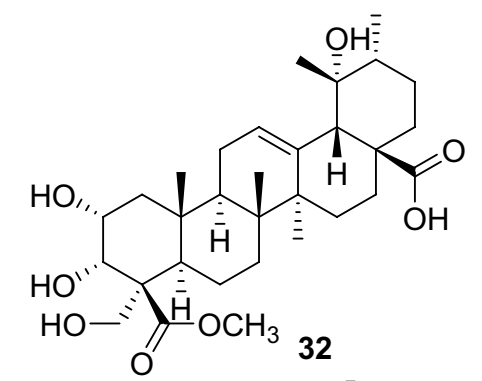

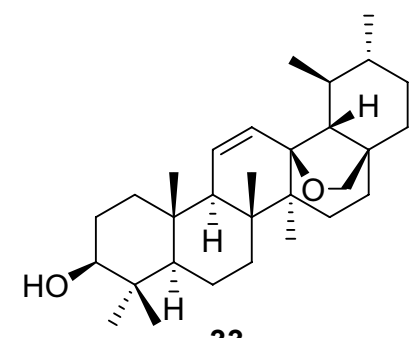

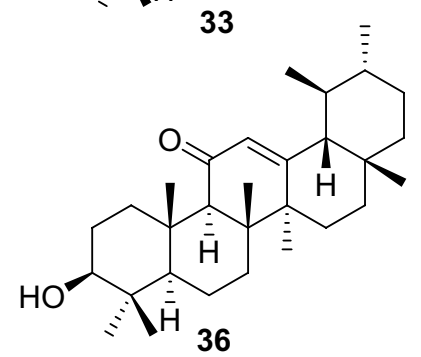

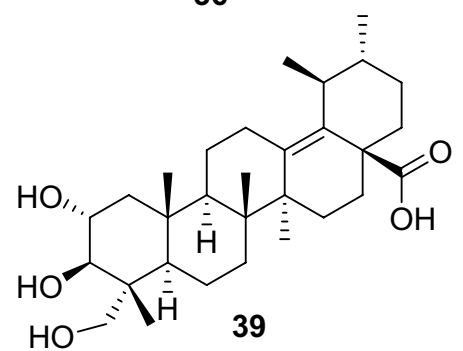

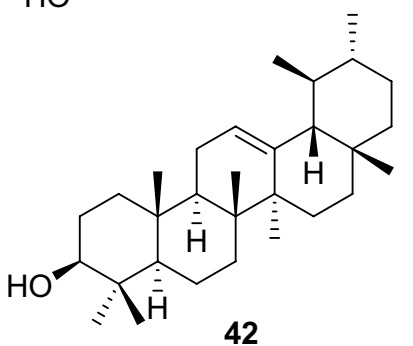

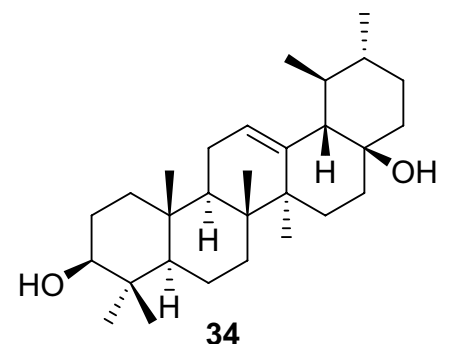

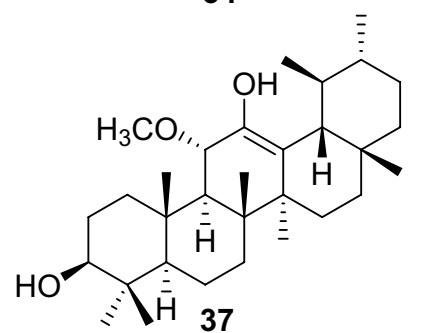

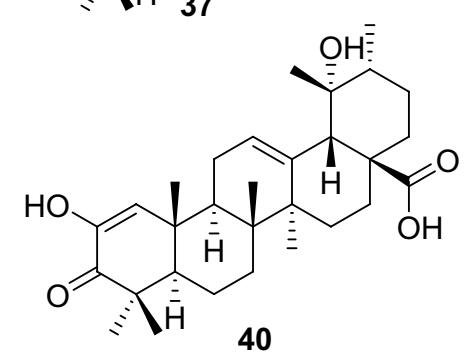

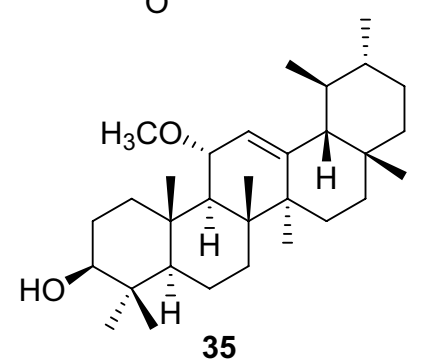

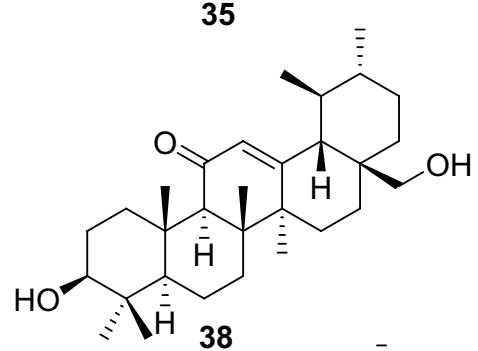

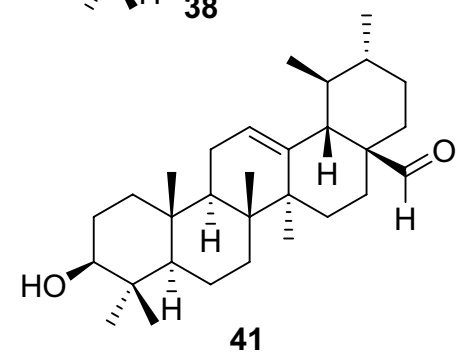

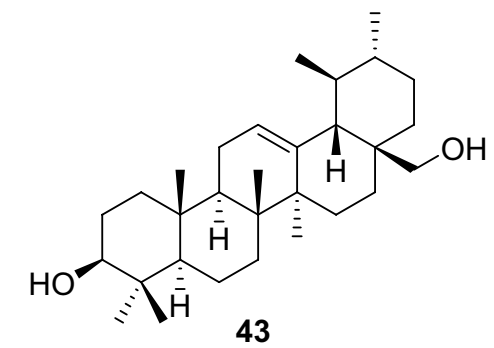

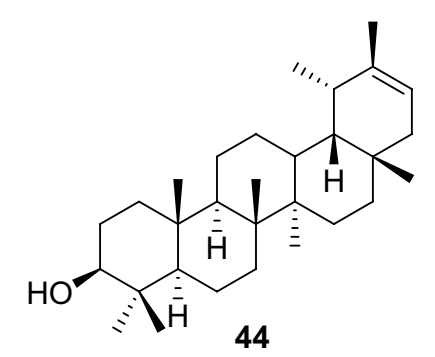

Fig. 2 (continued) 


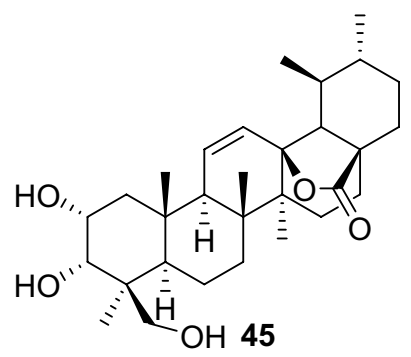

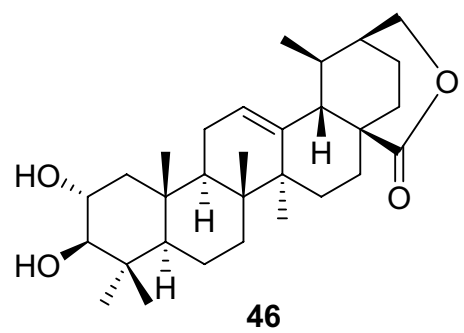

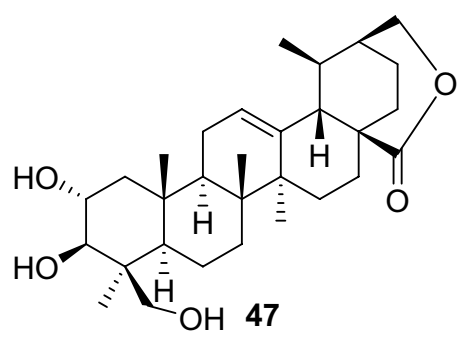

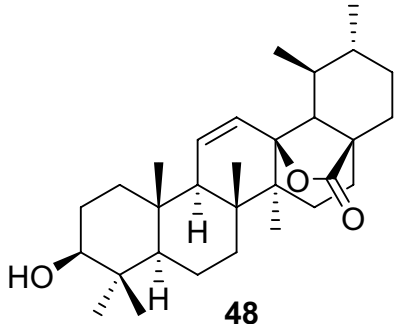

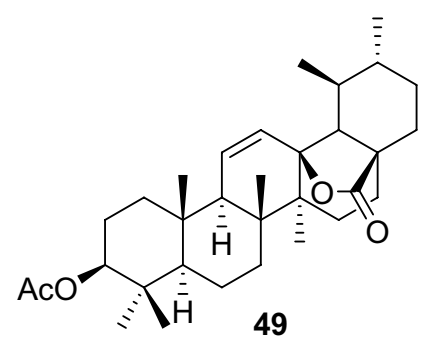<smiles>CC1=C2C3=CC[C@@H]4[C@](C)(CC[C@@]5(C)[C@H](C)[C@H](O)CC[C@]45C)[C@H]3CCC2(C(=O)O)CC[C@H]1C</smiles><smiles>CC1=C2C3=CC[C@@H]4[C@](C)(CC[C@@H]5[C@H](C)[C@H](O)[C@@H](O)C[C@]45C)[C@H]3CCC2(C(=O)O)CC[C@H]1C</smiles>

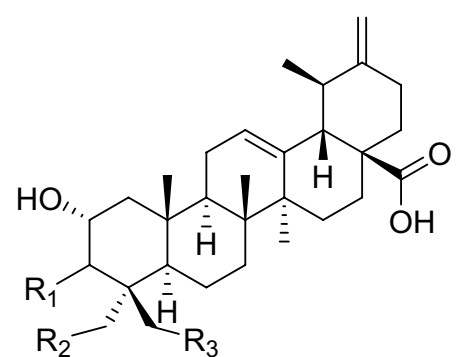

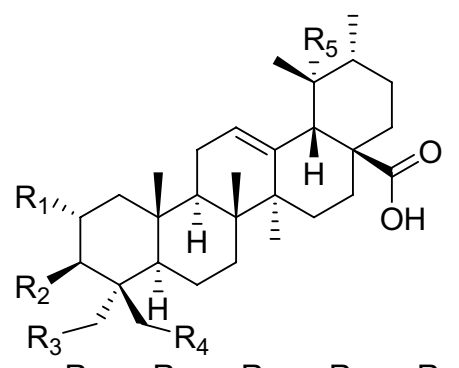

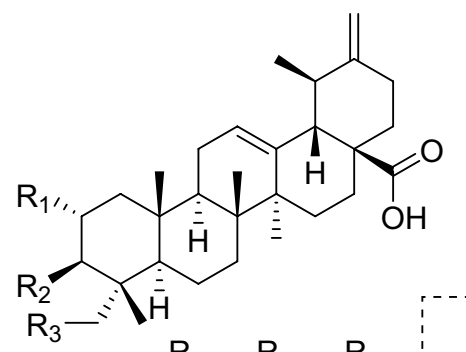
$\begin{array}{cll}\mathrm{R}_{1} & \mathrm{R}_{2} & \mathrm{R}_{3} \\ \mathbf{5 2} \alpha-\mathrm{OH} & \mathrm{OH} & \mathrm{H} \\ \mathbf{5 3} \beta-\mathrm{OH} & \mathrm{OH} & \mathrm{H} \\ \mathbf{5 4} \alpha-\mathrm{OH} & \mathrm{H} & \mathrm{OH} \\ \mathbf{5 5} \alpha-\mathrm{OH} & \mathrm{OH} & \mathrm{OH}\end{array}$ $\begin{array}{lllll}\mathrm{R}_{1} & \mathrm{R}_{2} & \mathrm{R}_{3} & \mathrm{R}_{4} & \mathrm{R}_{5}\end{array}$ $56 \mathrm{OH} \quad \mathrm{A} \quad \mathrm{H} \quad \mathrm{OH} \quad \mathrm{H}$ $\begin{array}{llllllllllllllll}57 & \mathrm{OH} & \mathrm{A} & \mathrm{H} & \mathrm{H} & \mathrm{OH}\end{array}$ $\begin{array}{lllllllllllllllll}58 & \mathrm{OH} & \mathrm{B} & \mathrm{H} & \mathrm{H} & \mathrm{OH}\end{array}$ $\begin{array}{lllllllllllllllll}59 & \mathrm{OH} & \mathrm{OH} & \mathrm{H} & \mathrm{H}\end{array}$ $60 \mathrm{OH} \mathrm{OH}$ A $\mathrm{Me} \quad \mathrm{H}$ $\begin{array}{lllll}61 \mathrm{~A} & \mathrm{OH} & \mathrm{OH} & \mathrm{H} & \mathrm{H}\end{array}$ $62 \mathrm{OH} \quad \mathrm{C} \quad \mathrm{OH} \quad \mathrm{H} \quad \mathrm{H}$ 63 OH $\mathrm{OH}$

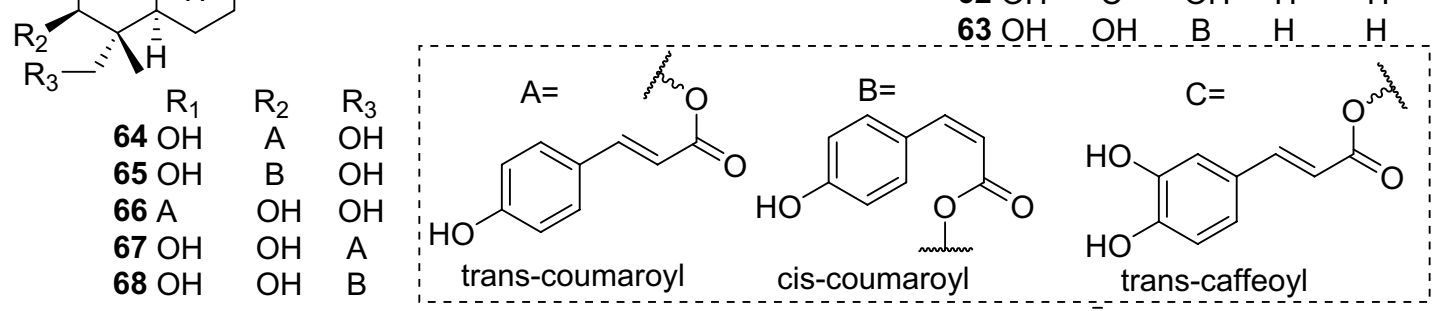

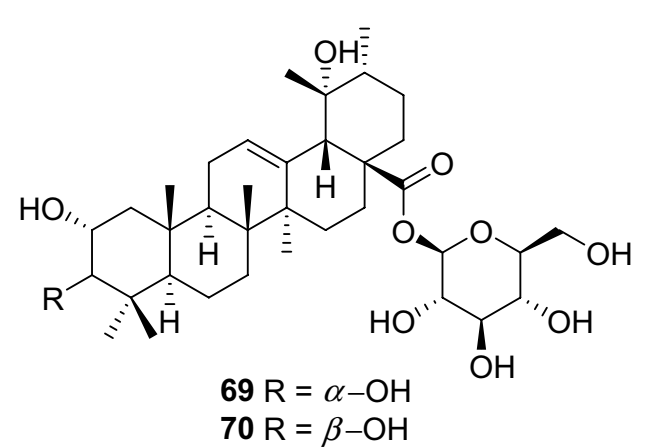

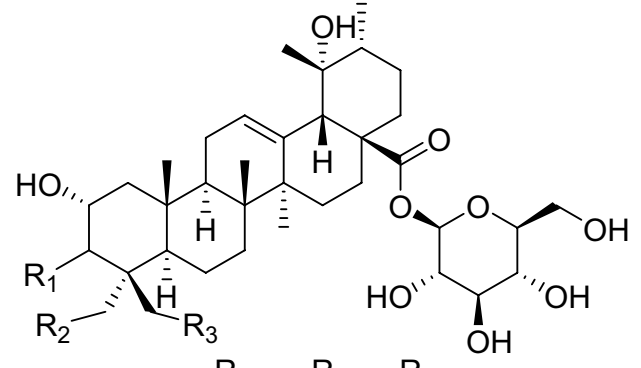
$\begin{array}{lll}\mathrm{R}_{1} & \mathrm{R}_{2} & \mathrm{R}_{3}\end{array}$ $71 \alpha-\mathrm{OH} \mathrm{H} \quad \mathrm{OH}$ $72 \beta-\mathrm{OH}$ OH $\mathrm{H}$

Fig. 2 (continued) 


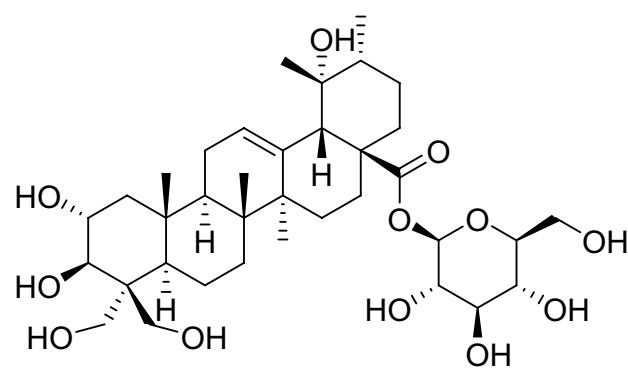

73

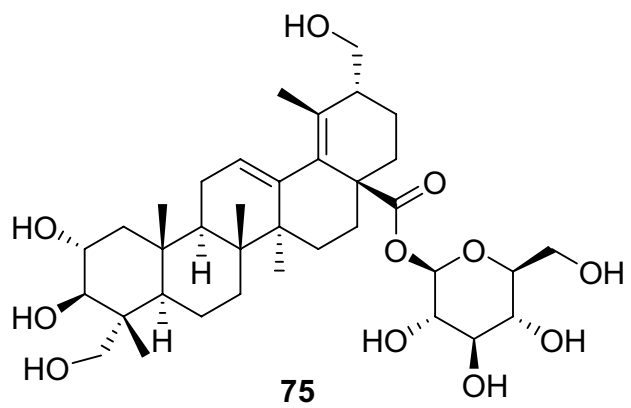

Fig. 2 (continued)

RAW246.7 cells also revealed that uvaol reduces mRNA expression and production of pro-inflammatory cytokines and mediators. These results indicating that uvaol is a prospective anti-inflammatory agent for colonic inflammation [27]. Guided by the hepatoprotective activity, the phytochemical study on the roots of $A$. chinensis led to the isolation of two new compounds $2 \alpha, 3 \beta$-dihydroxyurs- 12 -en28,30-olide 46, 2 $\alpha, 3 \beta, 24$-trihydroxyurs-12-en-28,30-olide 47 and $3 \beta$-hydroxyurs-12,18-dien-28-oic acid 50 [30]. Compounds 52-54 showed antifungal activity against $C$. musae at $3 \mu \mathrm{g} / \mathrm{mL}$ [31]. A new compound $2 \alpha, 3 \alpha, 23,24$-tetrahydroxyursa-12,20(30)-dien-28-oic acid $\mathbf{5 5}$ was isolated from the roots of $A$. chinensis Planch. It exhibited moderate antitumor activities against five human cancer cell lines (HepG2, A549, MCF-7, SK-OV-3, and HeLa) with $\mathrm{IC}_{50}$ values of $19.62 \pm 0.81,18.86 \pm 1.56,45.94 \pm 3.62$, $62.41 \pm 2.29$, and $28.74 \pm 1.07 \mu \mathrm{M}$, respectively [32]

Compounds 59-63 are actinidic acid derivatives with a phenylpropanoid unit that were identified as 3-O-trans$p$-coumaroylasiatic acid 59, 23-O-trans-p-coumaroylasiatic acid 60, actiniargupene $\mathrm{E}$ 61, actiniargupene $\mathrm{F}$ 62, and actiniargupene G 63 from the leaves of A. arguta. All the compounds showed inhibitory effects on $\alpha$-glucosidase activity. Among them compound 59 showed most potentially inhibitory activity on $\alpha$-glucosidase with an $\mathrm{IC}_{50}$ of

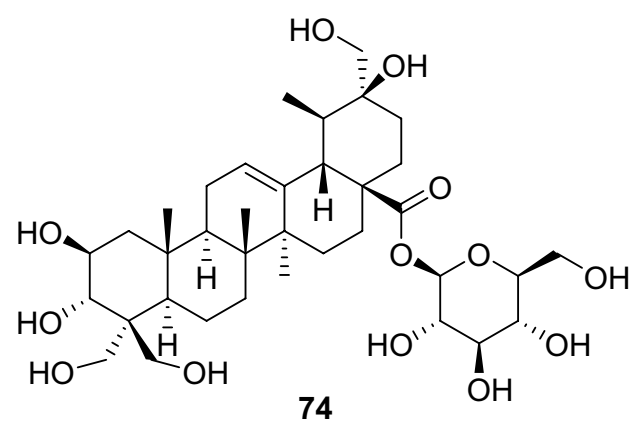

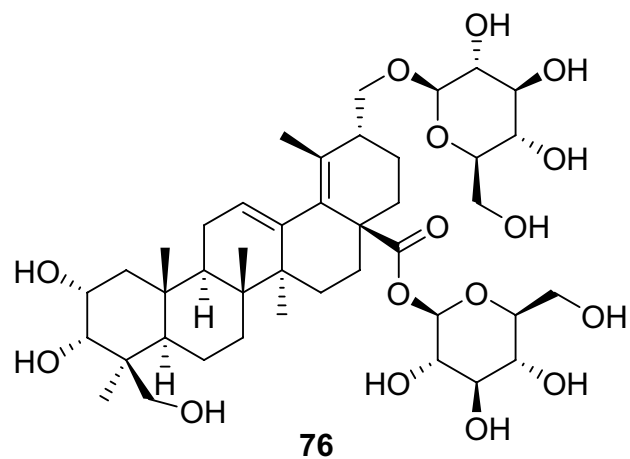

76

$81.3 \pm 2.7 \mu \mathrm{M}$, equal to that of the positive control (acarbose, $72.8 \pm 3.1 \mu \mathrm{M}$ ) [33]. The structure-activity relationship suggested that triterpenoids with a phenylpropanoid moiety exhibited more potent effects than those without such a unit [34]. Compound $\mathbf{7 1}$ showed potent cytotoxic activity against human SKVO3 and TPC-1 cancer cell lines with $\mathrm{IC}_{50}$ values of 10.99 and $14.34 \mu \mathrm{M}$, respectively [19, 35]. Compound $\mathbf{7 4}$ exhibited moderate cytotoxic activity against BEL-7402 and SMMC-7721 tumor cell lines [22]. Compounds 75 and 76 were isolated from roots of A. valvata Dunn. They exhibited moderate cytotoxic activity in vitro against BEL-7402 and SMMC-7721 tumor cell line [36].

\subsubsection{Oleanane Triterpenoids}

Oleanane-type triterpenoids also possessed a 6/6/6/6/6 pentacyclic carbon skeleton. Unlike ursane triterpenes, oleanane-type triterpenoids have two methyl groups at the C-20 position instead of each one at the C-19 and C-20, respectively. So far, a total of 24 oleanane-type triterpenoids have been identified from Actinidia plants (77-100, Fig. 3, Table 2). The most representative compound is oleanolic acid 77. It was found from callus tissue from the stems of A. arguta, together with $2 \alpha, 3 \beta$-dihydroxyolean-12-en-28-oic 
Table 1 Information of ursane triterpenoids from Actinidia plants

\begin{tabular}{ll}
\hline No. & Compound name \\
\hline 1 & $3 \beta$-Hydroxyurs-12-en-28-oic acid
\end{tabular}

3 3,24 -Dihydroxyurs-12-en-28-oic acid

$32 \alpha, 3 \beta$-Dihydroxyurs-12-en-28-oic acid

4 23-Hydroxyursolic acid

$5 \quad 2 \beta, 3 \beta$-Dihydroxyursolic acid

$6 \quad 2 \alpha, 3 \alpha$-Dihydroxyurs-12-en-28-oic acid

$72 \alpha, 3 \alpha, 24-$ Trihydroxyurs-12-en-28-oic acid

$82 \alpha, 3 \beta, 24$-Trihydroxy-urs-12-en-28-oic acid

$92 \alpha, 3 \beta, 23$-Trihydroxyurs-12-en-28-oic acid

$102 \alpha, 3 \alpha, 23$-Trihydroxyurs-12-en-28-oic acid

$112 \beta, 3 \alpha, 24$-Trihydroxy-urs-12-en-28-oic acid

$122 \beta, 3 \beta, 10$-Trihydroxy ursolic acid

$132 \alpha, 3 \beta, 14 \beta$-Trihydroxy ursolic acid

$142 \alpha, 3 \alpha, 19$-Trihydroxyurs-12-en-28-oic acid

$152 \beta, 3 \beta, 23$-Trihydroxyurs-12-en-28-oic acid

$162 \beta, 3 \alpha, 23$-Trihydroxyurs-12-en-28-oic acid

$172 \alpha, 3 \alpha, 23,24-T e t r a h y d r o x y u r s-12-$ en-28-oic acid

$182 \alpha, 3 \beta, 19 \alpha, 23$-Tetrahydroxyurs-12-en28 -oic acid

$192 \alpha, 3 \beta, 19 \alpha, 24-T e t r a h y d r o x y u r s-12$-en-28-oic acid

$202 \alpha, 3 \alpha, 19 \alpha, 24$-Tetrahydroxyurs-12-en-28-oic acid

$212 \alpha, 3 \beta, 23,24$-Tetrahydroxyurs-12-en-28-oic acid

$222 \alpha, 3 \beta, 6 \beta, 23$-Tetrahydroxyurs-12-en-28-oic acid

$232 \alpha, 3 \beta, 23,27-T e t r a h y d r o x y-12-e n-28$-ursolic acid

$242 \alpha, 3 \alpha, 19 \alpha, 23,24$-Pentahydroxy-urs-12-en28-oic acid

$252 \alpha, 3 \beta, 19 \alpha, 23,24$-Pentahydroxyurs-12-en28-oic acid

$26(2 \beta, 3 \alpha, 6 \alpha)-2,3,6,20,23,30$-Hexahydroxyurs12-en-28-oic acid

$272 \beta, 3 \beta$-Dihydroxy-23-oxours-12-en-28-oic acid

\section{A. arguta [37] \\ A. polygama [23] \\ A. polygama [23] \\ A. arguta [13]}

A. arguta [13]

A. sp [39]

A. chinensis [30]

A. eriantha [40]

A. eriantha Benth [41]

A. polygama [18]

A. polygama [18]

A. chrysantha [42]

A. polygama [18]

A. rufa [43]

A. sp [39]

A. sp [39]

A. chinensis [44]

A. eriantha [7]

A. chinensis Planch [32]

A. polygama [18]

A. chinensis Planch [32]

A. indochinensis Merr. Var [46] Roots

A. valvata [21]

A. chinensis Planch [32]

A. valvata Dunn [47]

A. deliciosa [48]

A. chinensis Planch [19]

A. rufa Planch ex miq [43]

A. valvata Dunn [22]

A. eriantha Benth [49]

Roots

Roots

Roots

Roots

Roots

Roots

Roots

Roots

Roots

Roots

Roots

Roots

Roots
Part

Roots

Bioactivity Refs.

Anticancer (in vitro) [8]

Inhibiting amylolytic enzyme (in vitro) [9]

Antidepressant and neuroprotective (in vitro) [10]

Downregulating thymic stromal lymphopoietin

(in vitro) [11]

Leaves

Fruit galls

Fruit galls Anti-metastation

Roots (In vitro) [38]

Anticancer (In vitro) [12]

Roots Anti-pancreatic lipase

(In vitro) [13]

Anticancer(In vitro) [14-16]

Anti-inflammatory

(in vitro) [17]

Fruit galls Against A549, LOVO, and HepG2 cell lines (in vitro) [19]

Fruit galls Against A549, LOVO, and HepG2 cell lines

Roots (in vitro) [19]

Fruit galls

Inhibiting tumor angiogenesis (in vitro) [45]

Against HepG2, A549, MCF-7, SK-OV-3, and HeLa cell lines (in vitro) [32]

Roots Against HepG2, A549, MCF-7, SK-OV-3, and HeLa cell lines (in vitro) [32]

Fruit galls Against A549, LOVO and HepG2 cell lines (in vitro) [20]

Leaves

Anti-tumor (in vitro) [21]

Against BEL-7402 and SMMC-7721 cells lines (in vitro) [22] 
Table 1 (continued)

\begin{tabular}{|c|c|c|c|c|}
\hline No. & Compound name & Species Refs. & Part & Bioactivity Refs. \\
\hline 28 & $\begin{array}{l}2 \alpha, 3 \alpha \text {-Dihydroxyurs-12-ene-24-al-28-oic } \\
\text { acid }\end{array}$ & A. polygama [18] & Fruit galls & \\
\hline 29 & $\begin{array}{l}2 \alpha, 3 \alpha, 24 \text {-Trihydroxyurs-12-ene-23,28-dioic } \\
\text { acid }\end{array}$ & A. polygama $[18]$ & Fruit galls & \\
\hline 30 & $3 \beta$-O-Acetylursolic acid & A. polygama [23] & Fruit galls & $\begin{array}{l}\text { Inhibiting PTP1B } \\
\text { (in vitro) [24] }\end{array}$ \\
\hline 31 & $\begin{array}{l}\text { 24-Acetyloxy-2 } 2 \alpha, 3 \alpha \text {-dihydroxyurs-12-en- } \\
28 \text {-oic acid }\end{array}$ & A. eriantha [7] & Roots & \\
\hline 32 & $\begin{array}{l}2 \alpha, 3 \beta, 19 \alpha \text {-Trihydroxyurs-12-en-23,28- } \\
\text { dioic,acid-23-methylester }\end{array}$ & A. chinensis Radix [50] & Roots & \\
\hline 33 & $3 \beta$-Hydroxy-13,28-epoxyurs-11-en-3-ol & A. kolomikta [51] & Rhizomes & \\
\hline 34 & 28 -Norurs-12-en-3 $\beta, 17 \beta$-diol & A. kolomikta [51] & Rhizomes & \\
\hline 35 & Triptohypol E & A. kolomikta [51] & Rhizomes & \\
\hline 36 & Neoilexonol & A. kolomikta [51] & Rhizomes & \\
\hline 37 & $11 \alpha$-Methoxyurs-12-ene-3 $\beta, 12$-diol & A. arguta [34] & Leaves & \\
\hline 38 & Ilelatifol A & A. arguta [34] & Leaves & \\
\hline 39 & $\begin{array}{l}(2 \alpha, 3 \beta)-2,3,23 \text {-Trihydroxyurs-13(18)-en-28- } \\
\text { oic acid }\end{array}$ & A. chinensis Planch [52] & Roots & \\
\hline 40 & Fupenzic acid & A. chinensis [25] & Root bark & Antiviral (in vitro) [25] \\
\hline 41 & Nrsolaldehyde & A. arguta [26] & Stems & \\
\hline 42 & $\alpha$-Amyrin & A. arguta [26] & Stems & \\
\hline 43 & Uvaol & A. arguta [26] & Stems & $\begin{array}{l}\text { Anti-inflammatory } \\
\text { (in vivo) [27] } \\
\text { Anticancer (in vitro) [28] } \\
\text { Wound healing (in vivo) [29] }\end{array}$ \\
\hline 44 & Pseudotaraxasterol & A. chinensis Planch [19] & Roots & \\
\hline 45 & $2 \alpha, 3 \alpha, 24$-Trihydroxyurs-11-en-13,28-olide & A. polygama [23] & Fruit galls & \\
\hline 46 & $2 \alpha, 3 \beta$-Dihydroxyurs-12-en-28,30-olide & A. chinensis [30] & Roots & \\
\hline 47 & $2 \alpha, 3 \beta, 24$-Trihydroxyurs-12-en-28,30-olide & A. chinensis [30] & Roots & \\
\hline 48 & Ehretiolide & A. kolomikta [51] & Rhizomes & \\
\hline 49 & $3 \beta$-Acetoxyurs-11-en-28-oic 13(28)-lactone & A. kolomikta [51] & Rhizomes & \\
\hline $\mathbf{5 0}$ & $3 \beta$-Hydroxyurs-12,18-dien-28-oic acid & A. chinensis [30] & Roots & \\
\hline 51 & $\begin{array}{l}(2 \alpha, 3 \beta, 4 \alpha) \text {-2,3-Dihydroxy-24-norursa- } \\
\text { 12,18-dien-28-oic acid }\end{array}$ & A. valvata Dunn [47] & Roots & \\
\hline 52 & $\begin{array}{l}2 \alpha, 3 \alpha, 23 \text {-Trihydroxy-12,20(30)-ursadien- } \\
28 \text {-oic acid }\end{array}$ & $\begin{array}{l}\text { A. polygama }[18] \\
\text { A. deliciosa }[31]\end{array}$ & $\begin{array}{l}\text { Fruit galls } \\
\text { Peels }\end{array}$ & Antifungal (in vitro) [31] \\
\hline 53 & $\begin{array}{l}2 \alpha, 3 \beta, 23 \text {-Trihydroxy-12,20(30)-ursadien- } \\
28 \text {-oic acid }\end{array}$ & A. deliciosa [31] & Peels & Antifungal (in vitro) [31] \\
\hline 54 & $\begin{array}{l}2 \alpha, 3 \alpha, 24 \text {-Trihydroxy-12,20(30)-ursadien- } \\
28 \text {-oic acid }\end{array}$ & A. deliciosa [31] & Peels & Antifungal (in vitro) [31] \\
\hline 55 & $\begin{array}{l}2 \alpha, 3 \alpha, 23,24-\text { Tetrahydroxyursa-12,20(30)- } \\
\text { dien-28-oic acid }\end{array}$ & A. chinensis Planch [32] & Roots & Anti-tumor (in vitro) [32] \\
\hline 56 & $\begin{array}{l}3 \beta \text {-Trans- } p \text {-coumaroyloxy- } 2 \alpha, 24 \text {-dihydroxy- } \\
\text { urs- } 12 \text { - en-28-oic acid }\end{array}$ & A. polygama [23] & Fruit galls & \\
\hline 57 & 3-O-Trans-p-coumaroyl tormentic acid & A. chinensis Radix [50] & Roots & \\
\hline 58 & 3-O-Cis-p-coumaroyl tormentic acid & A. chinensis Radix [50] & Roots & \\
\hline 59 & 3-O-Trans-p-coumaroylasiatic acid & A. polygama $[18]$ & Fruit galls & \\
\hline 60 & 23-O-Trans-p-coumaroylasiatic acid & A. arguta [34] & Leaves & Inhibiting $\alpha$-glucosidase (in vitro) [34] \\
\hline 61 & Actiniargupene E & A. arguta [34] & Leaves & Inhibiting $\alpha$-glucosidase (in vitro) [34] \\
\hline 62 & Actiniargupene $\mathrm{F}$ & A. arguta [34] & Leaves & Inhibiting $\alpha$-glucosidase (in vitro) [34] \\
\hline 63 & Actiniargupene $\mathrm{G}$ & A. arguta [34] & Leaves & Inhibiting $\alpha$-glucosidase (in vitro) [34] \\
\hline 64 & 3-O-Trans-p-coumaroyl actinidic acid & $\begin{array}{l}\text { A. arguta }[13] \\
\text { A. arguta }[34]\end{array}$ & $\begin{array}{l}\text { Roots } \\
\text { Leaves }\end{array}$ & \\
\hline
\end{tabular}


Table 1 (continued)

\begin{tabular}{|c|c|c|c|c|}
\hline No. & Compound name & Species Refs. & Part & Bioactivity Refs. \\
\hline 65 & 3-O-Cis-p-coumaroylactinidic acid & A. arguta [34] & Leaves & Inhibiting $\alpha$-glucosidase (in vitro) [34] \\
\hline 66 & Actiniargupene A & A. arguta [34] & Leaves & Inhibiting $\alpha$-glucosidase (in vitro) [34] \\
\hline 67 & Actiniargupene B & A. arguta [34] & Leaves & Inhibiting $\alpha$-glucosidase (in vitro) [34] \\
\hline 68 & Actiniargupene $\mathrm{C}$ & A. arguta [34] & Leaves & Inhibiting $\alpha$-glucosidase (in vitro) [34] \\
\hline 69 & (+)-Tormentoside & A. arguta [53] & Roots & \\
\hline 70 & $\begin{array}{l}\text { (+)-Euscaphic acid-28-O- } \beta \text {-D- } \\
\text { glucopyranoside }\end{array}$ & A. arguta [53] & Roots & \\
\hline 71 & $\begin{array}{l}2 \alpha, 3 \alpha, 19 \alpha, 24-T e t r a h y d r o x y u r s-12-e n-28 \text {-oic } \\
\text { acid } 28 \text {-O- } \beta \text {-D-glucopyranoside }\end{array}$ & A. chinensis Planch [19] & Roots & $\begin{array}{l}\text { Inhibiting SKVO3 and TPC-1 cancer cells } \\
\text { lines } \\
\text { (in vitro) [42] }\end{array}$ \\
\hline 72 & $\begin{array}{l}2 \alpha, 3 \beta, 19 \alpha, 23 \text {-Tetrahydroxyurs-12-en-28-oic } \\
\text { acid } 28 \text {-O- } \beta \text {-D-glucopyranoside }\end{array}$ & A. chinensis Radix [50] & Roots & \\
\hline 73 & $\begin{array}{c}2 \alpha, 3 \beta, 19 \alpha, 23,24-P e n t a h y d r o x y u r s-12 \text {-en- } \\
28 \text {-oic acid-28-O- } \beta \text {-D-glucopyranoside }\end{array}$ & A. rufa [35] & Roots & \\
\hline 74 & $\begin{array}{l}(2 \beta, 3 \alpha)-2,3,20,23,24,30 \text {-Hexahydroxyurs- } \\
\text { 12-en-28-oic acid O- } \beta \text {-D-glucopyranosyl } \\
\text { ester }\end{array}$ & A. valvata Dunn [22] & Roots & $\begin{array}{l}\text { Against BEL-7402 and SMMC-7721 tumor } \\
\text { cells lines (in vitro) [22] }\end{array}$ \\
\hline 75 & $\begin{array}{l}2 \alpha, 3 \beta, 23,30 \text {-Tetrahydroxyurs-12,18-diene- } \\
28 \text {-oic acid O- } \beta \text {-D-glucopyranosyl ester }\end{array}$ & A. valvata Dunn [36] & Roots & $\begin{array}{l}\text { Against BEL-7402 and SMMC-7721 tumor } \\
\text { cells lines (in vitro) [36] }\end{array}$ \\
\hline 76 & $\begin{array}{l}\text { 30-O- } \beta \text {-D-Glucopyranosyloxy- } 2 \alpha, 3 \alpha, 24- \\
\text { trihydroxyurs-12,18-diene-28-oic acid O- } \beta \text { - } \\
\text { D-glucopyranosyl ester }\end{array}$ & A. valvata Dunn [36] & Roots & $\begin{array}{l}\text { Against BEL-7402 and SMMC-7721 tumor } \\
\text { cells lines (in vitro) [36] }\end{array}$ \\
\hline
\end{tabular}

acid 78 [26]. Oleanolic acid $\mathbf{7 7}$ is abundant in nature and exhibits a wide range of biological activities including anti-inflammatory [54], anti-hypertension [55], anti-tumor [56, 57], neuroprotection [58], and anti-cholesterol activities [59]. Oleanolic acid was performed to test the effect on apoptosis and autophagy of SMMC-7721 Hepatoma cells. It can significantly inhibit the growth of liver cancer SMMC-7721 cells and induce autophagy and apoptosis [57]. Compound 78 also showed anti-tumor and anti-inflammatory activities [60, 61]. Lim et al. have demonstrated that $\mathbf{7 8}$ showed very strong anti-tumor-promoting activity with an $\mathrm{IC}_{50}$ of $0.1 \mathrm{mg} / \mathrm{mL}$ [60].

Bioassay- and ${ }^{1} \mathrm{H}$ NMR-guided fractionation of the methanol extract afforded two oleanolic acids of $2 \alpha, 3 \beta, 23-$ trihydroxyolean-12-en-28-oic acid $\mathbf{8 0}$ and $2 \alpha, 3 \alpha, 24$ trihydroxyolean-12-en-28-oic acid $\mathbf{8 1}$, showing antifungal activity against $C$. musae at $3 \mu \mathrm{g} / \mathrm{mL}$ [31]. The EtOAc extract of the roots of $A$. eriantha Benth exhibited potent growth inhibitory activity against SGC7901 cells, CNE2 cells and HUVECs cells. From which, compound 87 (3 $\beta, 23,24$-trihydroxyl-12-oleanen-28-oic acid) was identified [62]. Compound $\mathbf{8 8}$ was extracted from the roots bark of A. chinensis, which showed anti-viral activity [25]. A new triterpenoid $12 \alpha$-chloro- $2 \alpha, 3 \beta, 13 \beta, 23$-tetrahydroxyolean28-oic acid-13-lactone $\mathbf{8 9}$ was extracted from the roots of A. chinensis Planch (Actinidiaceae). It was tested for cytochrome P450 (CYPs) enzyme inhibitory activity in later years, which could significantly inhibit the catalytic activities of CYP3A4 to $<10 \%$ of its control activities $[19,52]$.

$3 \beta$-(2-Carboxybenzoyloxy) oleanolic acid 93 and spathodic acid-28-O- $\beta$-D-glucopyranoside 94 were extracted from the root bark of $A$. chinensis. The anti-phytoviral activity test indicated that $\mathbf{9 4}$ showed potent activity on TMV, and CMV with inactivation effect of $46.67 \pm 1.05$, and $45.79 \pm 2.23(100 \mathrm{mg} / \mathrm{L})$, compared to ningnanmycin with inactivation effect of $30.15 \pm 1.16$ and $27.18 \pm 1.02$ $(100 \mathrm{mg} / \mathrm{L})$ respectively [25]. 3 $\beta, 23$-Dihydroxy- -30-norolean-12,20(29)-dien-28-oic acid 98, 3 $\beta, 23$-dihydroxy1-oxo-30-norolean-12,20(29)-dien-28-oic acid 99, and $2 \alpha, 3 \alpha, 23,24$-tetrahydroxy-30-norolean-12,20(29)-dien28-oicacid $\mathbf{1 0 0}$ are three one-carbon-degraded oleanane triterpenoids that were identified from $A$. chinensis Radix for the first time [50].

\subsubsection{Lupane Triterpenoids}

Lupane triterpenoids possess a 6/6/6/6/5-fused carbon skeleton. Compared with ursane and oleanane triterpenoids, the number of lupane triterpenoids in the Actinidia 
Fig. 3 Structures of oleanane triterpenoids 77-100 from Actinidia plants

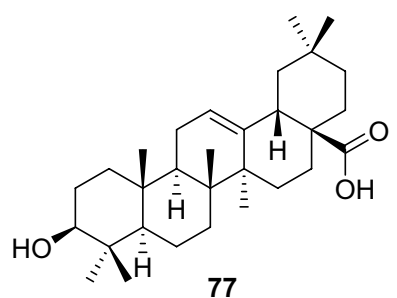

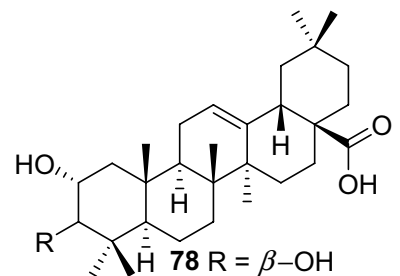

$79 \mathrm{R}=\alpha-\mathrm{OH}$

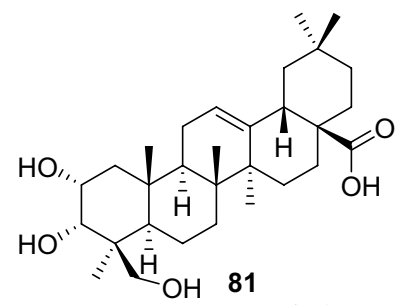

$\mathrm{OH}$

81

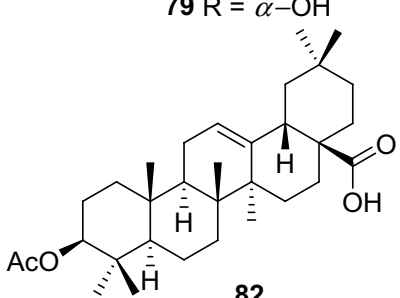

82

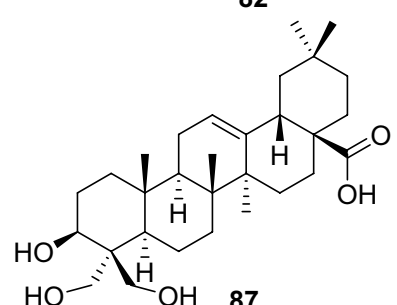

$\mathrm{HO}-\mathrm{H}_{\mathrm{OH}} 87$

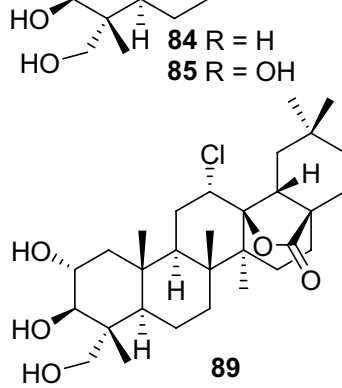

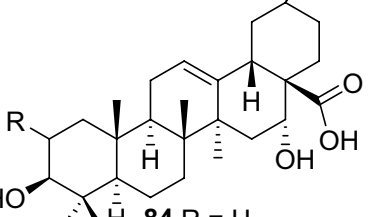

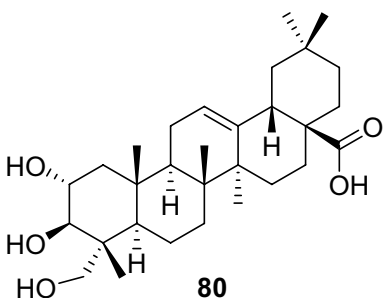

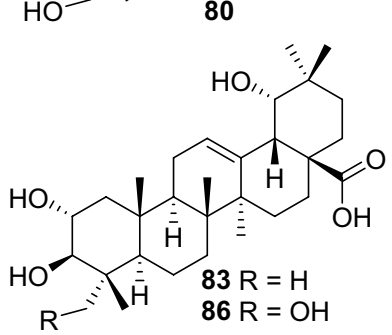

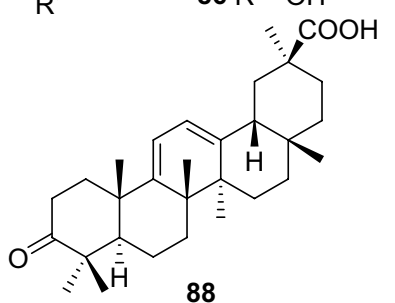

88

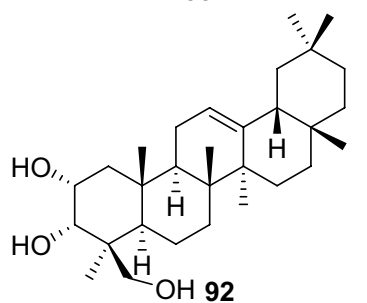

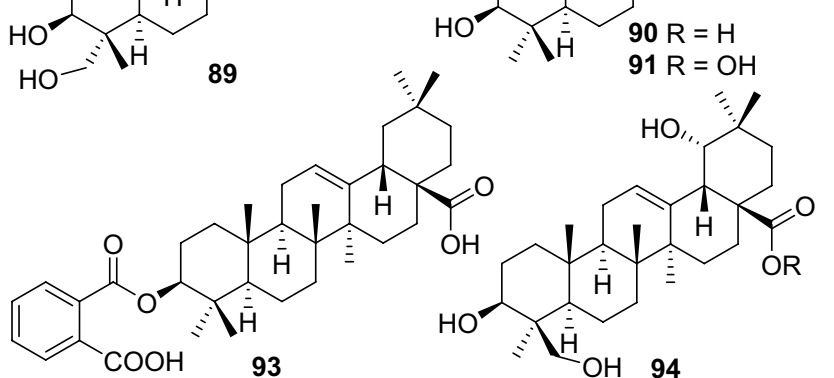

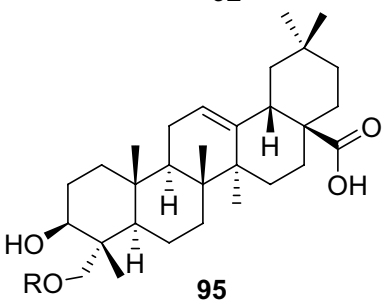

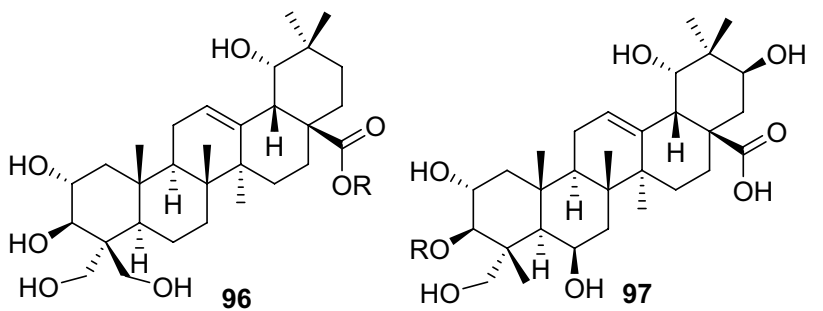<smiles>[R]CC1O[C@H](C)[C@@H](O)[C@H](O)[C@H]1O</smiles>

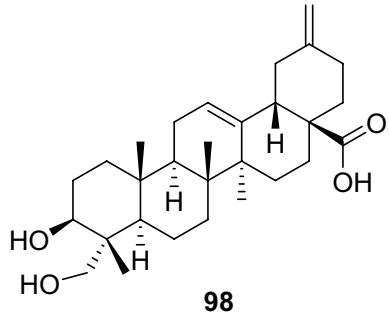<smiles>C=C1CCC2(C(=O)O)CC[C@]3(C)C(=CCC4[C@@]3(C)CC[C@@]3(C)[C@@H](C)[C@H](O)CC(=O)[C@]43C)C12C</smiles><smiles>C=C1CCC2(C(=O)O)CC[C@]3(C)C(=CCC4[C@@]5(C)C[C@H](O)[C@@H](O)C(CO)(CO)C5CC[C@]43C)C12CCO</smiles> 
Table 2 Information of oleanane triterpenoids from Actinidia plants

\begin{tabular}{|c|c|c|c|c|}
\hline No. & Compound name & Species Refs. & Part & Bioactivity Refs. \\
\hline 77 & Oleanolic acid & A. arguta $[26]$ & Stems & $\begin{array}{l}\text { Anti-inflammatory (in vitro) } \\
\text { [54] Anti-hypertension } \\
\text { (in vivo) [55] Anti-tumor } \\
\text { (in vitro) [56, 57] } \\
\text { Neuroprotection(in vitro) [58] } \\
\text { Anti-cholesterol(in vitro) [59] }\end{array}$ \\
\hline 78 & $2 \alpha, 3 \beta$-Dihydroxyolean-12-en-28-oic acid & A. arguta [26] & Stems & \\
\hline 79 & $2 \alpha, 3 \alpha$-Dihydroxyolean-12-en-28-oic acid & A. chinensis Planch [63] & Fruits & \\
\hline 80 & $2 \alpha, 3 \beta, 23$-Trihydroxyolean-12-en-28-oic acid & A. deliciosa [31] & Peels & Antifungal (in vitro) [31] \\
\hline 81 & $2 \alpha, 3 \alpha, 24$-Trihydroxyolean-12-en-28-oic acid & A. deliciosa [31] & Peels & Antifungal (in vitro) [31] \\
\hline 82 & $3 \beta$-O-Acetyloleanolic acid & $\begin{array}{l}\text { A. arguta }[64] \\
\text { A. chinensis }[45]\end{array}$ & $\begin{array}{l}\text { Stems } \\
\text { Roots }\end{array}$ & Anti-angiogenesis (in vitro) [45] \\
\hline 83 & $2 \alpha, 3 \beta, 19$-Trihydroxyolean-12-en-28-oic acid & A. chinensis [45] & Roots & Anti-angiogenesis (in vitro) [45] \\
\hline 84 & $\begin{array}{l}(3 \beta, 4 \alpha, 16 \alpha)-3,16,23 \text {-Trihydroxyolean-12- } \\
\text { en-28-oic acid }\end{array}$ & A. valvata Dunn [47] & Roots & \\
\hline 85 & $\begin{array}{l}(2 \beta, 3 \beta, 4 \alpha, 16 \alpha)-2,3,16,23 \text {-Tetrahydroxyolean-12-en- } \\
28 \text {-oic acid }\end{array}$ & A. valvata Dunn [47] & Roots & \\
\hline 86 & $2 \alpha, 3 \beta, 19 \alpha, 23$-Tetrahydroxyoleanolic acid & A. deliciosa $[65]$ & Roots & \\
\hline 87 & $3 \beta, 23,24$-Trihydroxyl-12-oleanen-28-oic acid & A. eriantha Benth [62] & Roots & Anti-angiogenesis (in vitro) [62] \\
\hline 88 & 9(11),12-Diene-30-oic acid & A. chinensis [25] & Roots bark & Anti-viral (in vitro) [25] \\
\hline 89 & $\begin{array}{l}\text { 12 } \alpha \text {-Chloro- } 2 \alpha, 3 \beta, 13 \beta, 23 \text {-tetrahydroxyolean-28-oic acid- } \\
\text { 13-lactone }\end{array}$ & A. chinensis Planch [19] & Roots & Anti-catalysis (in vitro) [43] \\
\hline 90 & $\beta$-Amyrin & A. arguta [26] & Stems & \\
\hline 91 & Erythrodiol & A. kolomikta [51] & Dried rhizomes & \\
\hline 92 & 12-Oleanene $2 \alpha, 3 \alpha, 24$-triol & A. macrosperma [66] & Roots & \\
\hline 93 & $3 \beta$-(2-Carboxybenzoyloxy) oleanolic acid & A. chinensis [25] & Root bark & Anti-phytoviral (in vitro) [25] \\
\hline 94 & Spathodic acid-28-O- $\beta$-D-glucopyranoside & A. chinensis [25] & Root bark & Anti-phytoviral (in vitro) [25] \\
\hline 95 & Oleanolic acid-23-O- $\beta$-D-glucopyranoside & A. eriantha Benth [62] & Roots & Anti-angiogenesis (in vitro) [62] \\
\hline 96 & $\begin{array}{l}2 \alpha, 3 \beta, 19 \alpha, 23,24-P e n t a h y d r o x y-12 \text {-oleanen } 28 \text {-oic acid } \\
28 \text { - } \beta \text {-D-glucopyranosyl }\end{array}$ & A. chinensis Radix [50] & Roots & \\
\hline 97 & $\begin{array}{l}\text { 3-O- } \beta \text {-D-Glucopyranosyl- } 2 \alpha, 3 \beta, 6 \beta, 19 \alpha, 21 \beta, 23 \text { - } \\
\text { hexahydroxylolean-12-en-28-oic acid }\end{array}$ & A. kolomikta [67] & Leaves & \\
\hline 98 & $\begin{array}{l}\text { 3 } \beta, 23 \text {-Dihydroxy-30-norolean-12,20(29)- } \\
\text { dien-28-oic acid }\end{array}$ & A. chinensis Radix [50] & Roots & \\
\hline 99 & $\begin{array}{l}\text { 3 } \beta, 23 \text {-Dihydroxy-1-oxo-30-norolean- } \\
\text { 12,20(29)-dien-28-oic acid }\end{array}$ & A. chinensis Radix [50] & Roots & \\
\hline 100 & $\begin{array}{l}2 \alpha, 3 \alpha, 23,24 \text {-Tetrahydroxy-30-norolean- } \\
12,20(29) \text {-dien-28-oic acid }\end{array}$ & A. chinensis Radix [50] & Roots & \\
\hline
\end{tabular}

plants is much smaller, only four related compounds have been identified (101-104, Fig. 4, Table 3). Three of them (101-103) were identified from the rhizomes of A. kolomikta [51]. Betulinic acid $\mathbf{1 0 1}$ is one of the most representative compound of lupane triterpenes, it has been extensively studied in recent years based on the wide biological activities including anti-inflammatory, antitumor, anti-HIV, anti-diabetic and antimalarial activities [68-73].
Much attention as a molecular target about protein tyrosine phosphatase 1B had been paid to the treatment of insulin resistance diseases because of its critical roles in negatively regulating insulin- and leptin-signaling cascades. Betulinic acid showed significant PTP1B inhibitory activity, with $\mathrm{IC}_{50}$ values of $3.5 \mu \mathrm{M}$ [24]. 


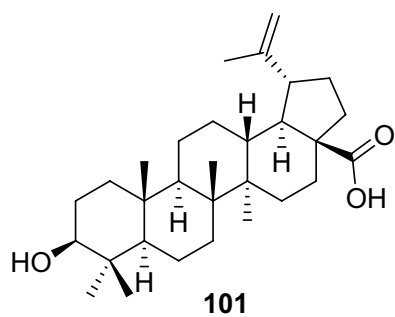

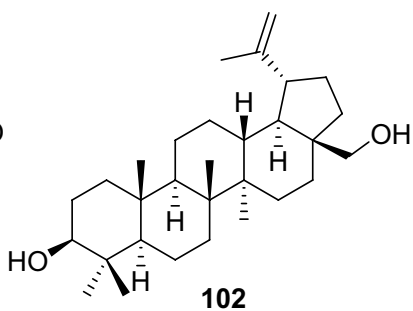

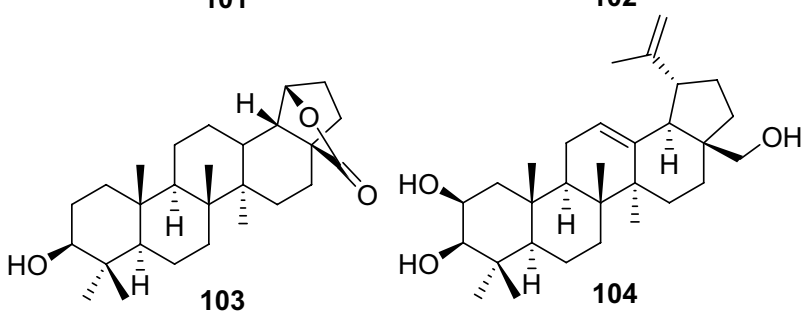

Fig. 4 Structures of lupane triterpenoids 101-104 from Actinidia plants

\subsubsection{Other Terpenoids}

A total of 19 other terpenoids including iridoids, diterpenoids, and their glycosides have been found from Actinidia plants (105-123, Fig. 5, Table 4). None of these compounds have good biological activities, only compound $\mathbf{1 2 0}$ showed certain anti-angiogenesis activity [77].

\subsection{Steroids}

$\beta$-Sitosterol 124 is a very normal phytosterol almost distributed in all plants. Eight phytosterols have been obtained from the Actinidia plants (124-131, Fig. 6, Table 5). Pharmacological studies on these steroids have demonstrated that $\beta$-sitosterol showed various bioactivities including anti-inflammatory, anti-cancer, antimicrobial and anti-diabetic properties [81-88]. A study suggested that $\beta$-sitosterol may serve as a potential therapeutic in the treatment of acute organ damages [82].

In addition to phytosterols, seven normal ergosterols (132-137, Fig. 6, Table 5) were obtained from peel or rhizomes of kiwifruit plants. It is well known that ergosterols should be fungal products. Compounds 132-137 may be produced by fungal infected kiwifruit plants.

\subsection{Phenols}

\subsubsection{Catechins and Epicatechins}

A total of 16 related compounds $(\mathbf{1 3 9}-\mathbf{1 5 4})$ have been obtained from kiwifruit plants (Fig. 7, Table 6). Compounds 148 and 149 possessed a novel structure featuring with a pyrrolidin-2-one substituent at C-6 and C-8, respectively. Compounds 152 and $\mathbf{1 5 3}$ were two sulfur-containing catechins that was rare in nature. Pharmacological studies have revealed that (+)-catechin $\mathbf{1 3 9}$ and (-)-epi-catechin $\mathbf{1 4 0}$ showed nitric oxide inhibitory activity in LPS stimulated RAW 264.7 cell with $\mathrm{IC}_{50}$ values of 26.61 and $25.30 \mu \mathrm{g} /$ $\mathrm{mL}$, respectively [53, 91]. Compound 147 showed moderate radical scavenging and antioxidant capabilities by measuring their capacity to scavenge DPPH and anion superoxide radical and to reduce a Mo(VI) salt [89]. Two new flavan3-ols, 6-(2-pyrrolidinone-5-yl)-(-)-epicatechin 148 and 8-(2-pyrrolidinone-5-yl)-(-)-epicatechin 149, as well as proanthocyanidin B-4 150, were isolated from an EtOAcsoluble extract of the roots of $A$. arguta. The isolates were tested in vitro for their inhibitory activity on the formation of advanced glycation end products (AGEs). All of them exhibited significant inhibitory activity against AGEs formation with $\mathrm{IC}_{50}$ values ranging from 10.1 to $125.2 \mu \mathrm{M}$ [92].

\subsubsection{Flavones, Isoflavones, and Flavonols}

A total of 48 flavone derivatives have been identified from kiwifruit plants, most of which are glycosides (155-202, Fig. 8, Table 7). Pharmacological studies indicated that these compounds, particularly kaempferol and its derivative, had a wide range of biological activities including antiproliferation, antioxidation, anti-inflammation, anticancer, anti-free radical, and neuroprotection activities [96-99]. Kaempferol 157 was found to prevent neurotoxicity by several ways
Table 3 Information of lupane triterpenoids from Actinidia Plants

\begin{tabular}{lllll}
\hline No. & Compound name & Species Refs. & Part & Bioactivity Refs. \\
\hline $\mathbf{1 0 1}$ & Betulinic acid & A. kolomikta [51] & Rhizomes & $\begin{array}{l}\text { Anti-inflammatory } \\
\text { (in vitro) [68] } \\
\text { Antitumor(in vitro) [69] } \\
\end{array}$ \\
& & & $\begin{array}{l}\text { Anti-HIV(in vitro) [72] } \\
\text { Anti-diabetic(in vivo) [73] }\end{array}$ \\
$\mathbf{1 0 2}$ & Betulin & A. kolomikta [51] & Rhizomes & Anti-tumor(in vitro) [74-76] \\
$\mathbf{1 0 3}$ & Diospyrolide & A. kolomikta [51] & Rhizomes & \\
$\mathbf{1 0 4}$ & Lupa-12,20(30)-diene- & A. deliciosa [48] & Roots & \\
& $2 \beta, 3 \beta, 28$-triol & & & \\
\hline
\end{tabular}


<smiles>[R]C1COC(=O)[C@@H]2[C@@H](C)CC[C@@H]12</smiles>

$105 \beta$-Me

$106 \alpha-\mathrm{Me}$<smiles>C[C@H]1CC[C@@H]2[C@H]1COC(=O)[C@@H]2C</smiles>

107<smiles>CC1=C2C(=O)OC[C@@H](C)[C@H]2CC1</smiles>

108<smiles>[R]C1C(=O)OCC2=C(C)CC[C@H]21</smiles>

$109 \beta$-Me

$110 \alpha$-Me<smiles>[R]C1C(=O)O[C@]2(C)C(C)=CC[C@@H]12</smiles>

$111 \beta$-Me

$112 \alpha-\mathrm{Me}$<smiles>CC1=C2C(=O)OC[C@@H](C)[C@H]2CC1</smiles><smiles>[R]C1COC(=O)[C@@H]2[C@@H](C)CC[C@H]12</smiles><smiles>[R]C1COC(=O)C2C(C)CCC12</smiles><smiles>C=C1COC(C)C[C@H]1C</smiles><smiles>C/C=C\C1C(C)=CC(=O)CC1(C)C</smiles><smiles>C=CC(C)O[C@H]1O[C@H](CO)[C@@H](O)[C@H](O)[C@H]1O</smiles>

120<smiles>C[C@H](O)/C=C/[C@@]1(O)C(C)(C)C[C@@H](O[C@@H]2O[C@H](CO)[C@@H](O)[C@H](O)[C@H]2O)C[C@]1(C)O</smiles><smiles>C[C@@H]1CC[C@H](/C=C/O[C@@H]2O[C@H](CO[C@@H]3O[C@H](CO)[C@@H](O)[C@H](O)[C@H]3O)[C@@H](O)[C@H](O)[C@H]2O)[C@H]1C=O</smiles><smiles>CC1=C(C=O)[C@H](/C=C/O[C@@H]2O[C@H](CO[C@@H]3O[C@H](CO)[C@@H](O)[C@H](O)[C@H]3O)[C@@H](O)[C@H](O)[C@H]2O)CC1</smiles>

Fig. 5 Structures of other terpenoids $\mathbf{1 0 5}-\mathbf{1 2 3}$ from Actinidia plants

Table 4 Information of other triterpenoids from Actinidia plants

\begin{tabular}{|c|c|c|c|c|}
\hline No. & Compound name & Species Refs. & Part & $\begin{array}{l}\text { Bioactivity } \\
\text { Refs. }\end{array}$ \\
\hline 105 & Dihydroepinepetalactone & A. polygama [78] & Fresh fruits & \\
\hline 106 & Isodihydroepinepetalactone & A. polygama [78] & Fresh fruits & \\
\hline 107 & Isoepiiridomyrmecin & A. polygama [78] & Fresh fruits & \\
\hline 108 & Isoneonepetalactone & A. polygama [78] & Fresh fruits & \\
\hline 109 & Dehydroiridomyrmecin & A. polygama [78] & Fresh fruits & \\
\hline 110 & Isodehydroiridomyrmecin & A. polygama [78] & Fresh fruits & \\
\hline 111 & Actinidialactone & A. polygama [78] & Fresh fruits & \\
\hline 112 & Isoactinidialactone & A. polygama [78] & Fresh fruits & \\
\hline 113 & Neonepetalactone & A. polygama [78] & Fresh fruits & \\
\hline 114 & Dihydronepetalactone & A. polygama [78] & Fresh fruits & \\
\hline 115 & Isodihydronepetalactone & A. polygama [78] & Fresh fruits & \\
\hline 116 & Iridomyrmecin & A. polygama [78] & Fresh fruits & \\
\hline 117 & Isoiridomyrmecin & A. polygama [78] & Fresh fruits & \\
\hline 118 & Matatabiether & A. polygama [79] & Leaves and galls & \\
\hline 119 & $\begin{array}{l}(R)-1,2,6,7,8,9-\text { Hexahy- } \\
\text { dro-10-hydroxy-1,6,6- } \\
\text { trimethylphenanthro[1,2-b]furan- } \\
\text { 5,11-dione }\end{array}$ & A. valvataDunn [47] & Roots & \\
\hline 120 & $\begin{array}{l}(6 R, 7 E, 9 S) \text {-6,9-Hydroxy- } \\
\text { megastigman-4,7-dien-3-one-9-O- } \\
\beta \text {-glucopyranoside }\end{array}$ & A. eriantha Benth [62] & Roots & $\begin{array}{l}\text { Anti-angiogen- } \\
\text { esis (in vitro) } \\
\text { [62] }\end{array}$ \\
\hline 121 & Kiwiionoside & A. chinensis [80] & Fresh leaves & \\
\hline 122 & Iridodialo- $\beta$-D-gentiobioside & A. polygama [77] & Leaves & \\
\hline 123 & Dehydroiridodialo- $\beta$-D-gentiobioside & A. polygama [77] & Leaves & \\
\hline
\end{tabular}




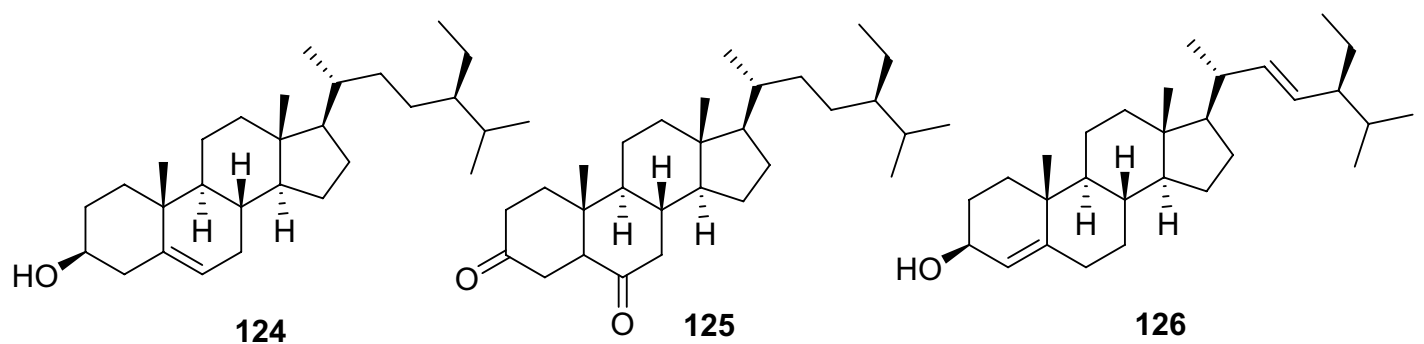

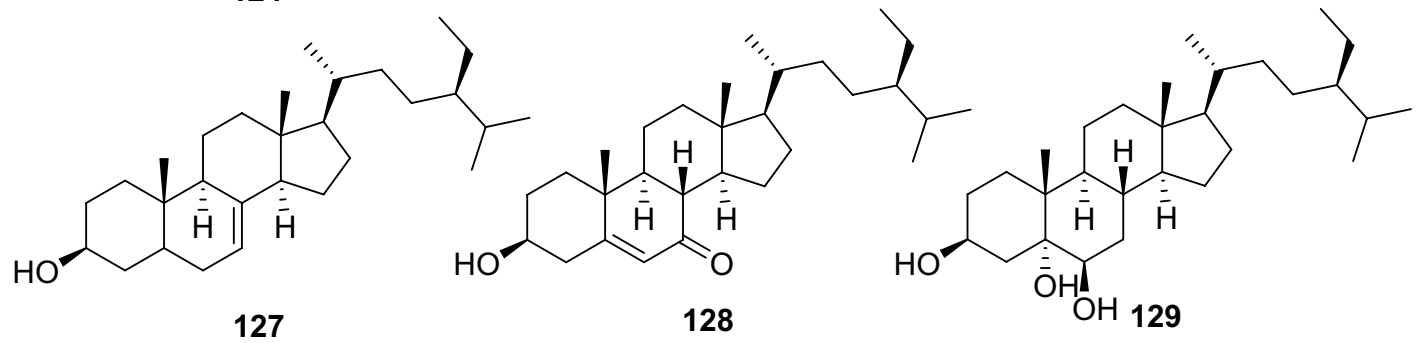<smiles>CCC(/C=C/[C@H](C)C1CCC2[C@H]3CC=C4C[C@@H](O)CC[C@]4(C)C3CC[C@@]12C)C(C)C</smiles><smiles>CCC(CC[C@@H](C)C1CCC2[C@H]3CC=C4C[C@@H](O[C@@H]5O[C@H](CO)[C@@H](O)[C@H](O)[C@H]5O)CC[C@]4(C)[C@H]3CC[C@@]12C)C(C)C</smiles><smiles>CC(C)[C@H](C)/C=C/[C@H](C)[C@H]1CCC2C3=CC=C4C[C@@H](O)CC[C@]4(C)[C@H]3CC[C@]21C</smiles>

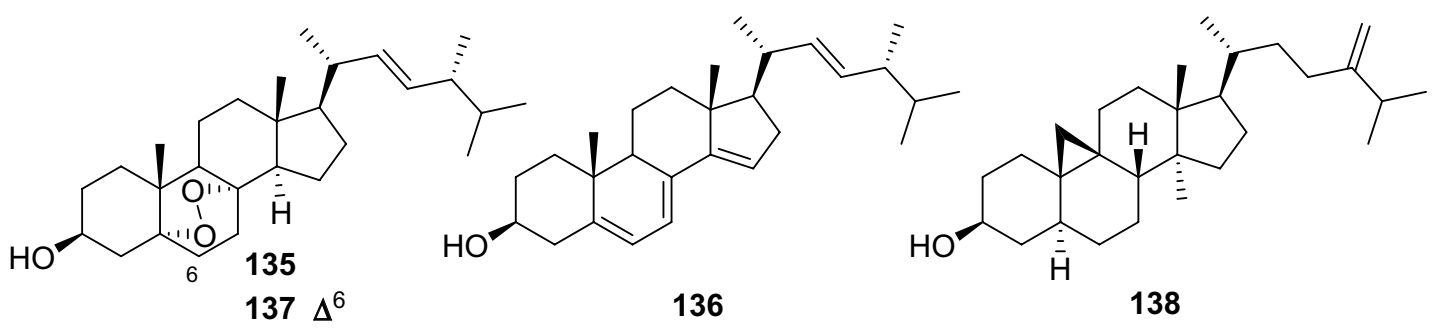

Fig. 6 Structures of steroids 124-138 from Actinidia plants

which was able to completely block $N$-methyl-D-aspartate (NMDA)-induced neuronal toxicity and potently inhibited MAO (monoamine oxidase) with the $\mathrm{IC}_{50}$ of $0.8 \mu \mathrm{M}$ [99]. Two novel flavonoids $\mathbf{1 7 1}$ and $\mathbf{1 7 2}$ were separated from the leaves of A. valvata Dunn. They exhibited dose-dependent activity in scavenging 1,1-diphenyl-2-picrylhydrazyl (DPPH) free radicals, superoxide anion radicals, and hydroxyl radicals, and inhibited lipid peroxidation of mouse liver homogenate in vitro [100]. Compounds 178 [101] and 179 [102] were two new compounds obtained from the leaves of A. kolomikta. The latter was screened for its protective effect on human erythrocytes against AAPH-induced hemolysis, 
Table 5 Information of steroids from Actinidia plants

\begin{tabular}{|c|c|c|c|c|}
\hline No. & Compound name & Species Refs. & Part & Bioactivity Refs. \\
\hline 124 & $\beta$-Sitosterol & A. eriantha [7] & Roots & $\begin{array}{l}\text { Anti-inflammatory } \\
\text { (In vivo) [84] } \\
\text { Anticancer (in vitro) [85] } \\
\text { Anti-microbial } \\
\text { (in vitro) [86] } \\
\text { Anti-diabetic } \\
\text { (In vivo) [88] }\end{array}$ \\
\hline 125 & Stigmast-3,6-dione & A. chrysantha [42] & Roots & \\
\hline 126 & Masterol & A. deliciosa [89] & Peels & \\
\hline 127 & Stigmast-7-en-3 $\beta$-ol & A. deliciosa [89] & Peels & \\
\hline 128 & $3 \beta$-Hydroxystigmast-5-en-7-one & A. chinensis Planch [52] & Roots & \\
\hline 129 & Sitostanetriol & A. kolomikta [51] & Dried rhizomes & \\
\hline 130 & (24R)-Stigmast-4-en-3-one & A. kolomikta [51] & Dried rhizomes & \\
\hline 131 & Stigmasterol & A. arguta [26] & Stems & $\begin{array}{l}\text { Anti-inflammatory } \\
\text { (in vitro) [90] }\end{array}$ \\
\hline 132 & Daucosterol & $\begin{array}{l}\text { A. eriantha }[40] \\
\text { A. chinensis }[44]\end{array}$ & $\begin{array}{l}\text { Roots } \\
\text { Roots }\end{array}$ & \\
\hline 133 & Campesterol & A. deliciosa [89] & Peels & \\
\hline 134 & Ergosterol & A. deliciosa [89] & Peels & \\
\hline 135 & Ergost-22-en-3-ol & A. deliciosa [89] & Peels & \\
\hline 136 & 5,7,14,22-Ergostatetraen-3 $\beta$-ol & A. deliciosa [89] & Peels & \\
\hline 137 & 5,8-Epidioxyergosta-6,22-dien-3 $\beta$-ol & A. kolomikta [51] & Dried rhizomes & \\
\hline 138 & 24-Methylene-pollinastanol & A. kolomikta [51] & Dried rhizomes & \\
\hline
\end{tabular}

which could slow the hemolysis induced by AAPH [102]. Eerduna et al. evaluated the effects of compound $\mathbf{1 8 2}$ on acute myocardial infarction in rats, the groups treated with 182 showed a dose-dependent reduction in myocardial infarct size model, markedly inhibited the elevation of the activity of creatine kinase, troponin $\mathrm{T}$ level, and the content of malondialdehyde induced by AMI [103]. Compound 182 also showed a capacity to increase the activities of superoxide dismutase, catalase, and endothelial nitric oxide synthase [104]. Lim et al. tested the DPPH radical scavenging activity and nitric oxide production inhibitory activity in IFN- $\gamma$, LPS stimulated RAW 264.7 cell of quercetin $\mathbf{1 8 5}$, quercetin-3-O$\beta$-D-glucoside 186, and quercetin 3-O- $\beta$-D-galactoside 193 with $\mathrm{IC}_{50}$ value of $20.41,18.23$, and $30.46 \mu \mathrm{g} / \mathrm{mL}$, respectively [91].

\subsubsection{Xanthones}

Three xanthones were isolated from n-butyl alcohol fraction of A. arguta (Sieb. \& Zucc) Planch. ex Miq and identified as 2 - $\beta$-D-glu-1,3,7-trihydrogen xanthone 203, 7-O-[ $\beta$-D-xylose- $(1 \rightarrow 6)-\beta$-D-glucopyranoside]1,8-dihydroxy-3-methoxy xanthone $\mathbf{2 0 4}$, and
1-O-[ $\beta$-D-xylose- $(1 \rightarrow 6)$ - $\beta$-D-glucopyranside] - 8 -hydroxy3,7-dimethoxy xanthone 205 (Fig. 9, Table 8). They were isolated from this plant for the first time [108]. Compound 203 showed extensive biological activities, including inhibiting $\alpha$-Glycosidase, NO production inhibition and NF- $\kappa \mathrm{B}$ inhibition and PPAR activation [116, 117]. It has been demonstrated the inhibitory effects on NF- $\kappa \mathrm{B}$ transcriptional activation in HepG2 cells stimulated with TNF $\alpha$ with an $\mathrm{IC}_{50}$ value of $0.85 \pm 0.07 \mu \mathrm{M}$, which was more potent than the positive control of sulfasalazine $\left(\mathrm{IC}_{50}=0.9 \mu \mathrm{M}\right)$ [118].

\subsubsection{Anthocyanins}

Five anthocyanins were obtained from the flesh of larger fruit of A. deliciosa and A. chinensis and identified as delphinidin 3-galactoside 206, cyanidin 3-galactoside 207, cyanidin 3-glucoside 208, delphinidin 3-[2-(xylosyl)galactoside] 209, and cyanidin 3-[2-(xylosyl)galactoside] 210, respectively (Fig. 9, Table 8) [119]. Cyanidin 3-glucoside 208 exhibited a wide range of pharmacological activities including anti-inflammatory, neuroprotective, anti-cancer, and antioxidant activities [120-124]. 


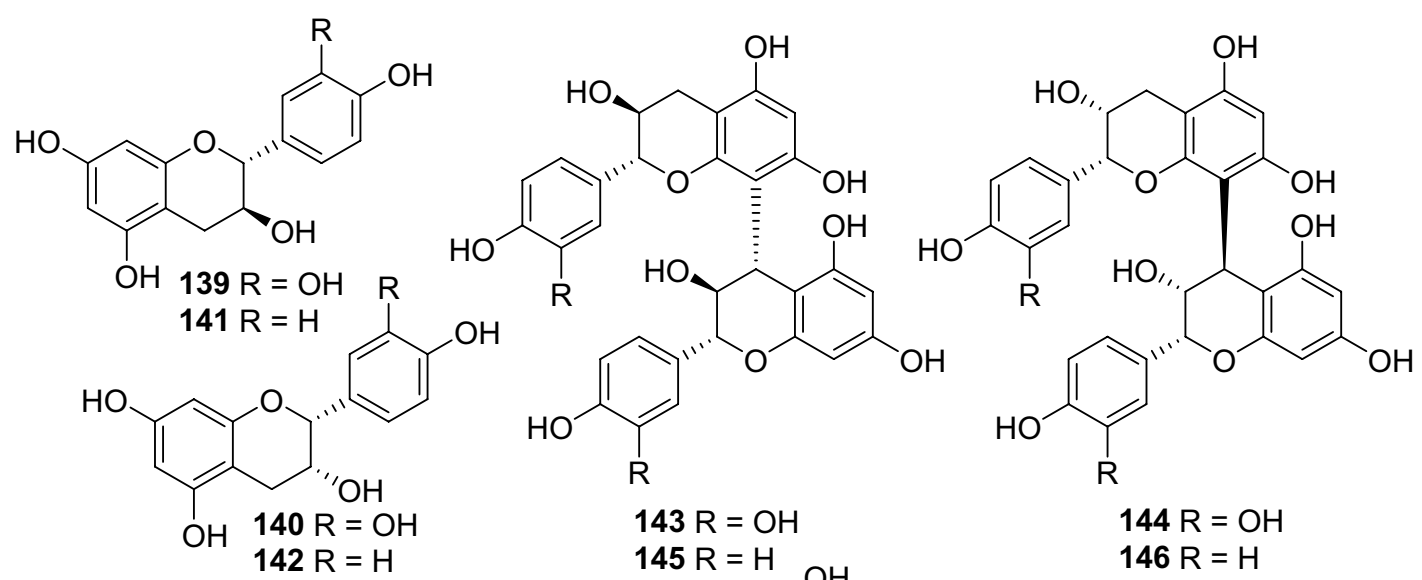<smiles>[Z16]C[C@H]1Cc2c(O)cc(O)cc2O[C@H]1c1cc(O)c(O)c(O)c1</smiles><smiles>CC(C)(C)SCc1ccccc1</smiles><smiles>CC(C)(C)c1cc([C@H]2Oc3ccc(O)c(O)c3[C@H](c3c(O)cc(O)c4c3O[C@H](c3ccc(O)c(O)c3)[C@H](O)C4c3c(O)cc(O)c4c3O[C@@H](c3ccc(O)c(O)c3)[C@H](O)C4)[C@H](O)C2c2c(O)cc(O)c3c2O[C@H](c2ccc(O)c(O)c2)[C@H](O)C3SCc2ccccc2)cc(O)c1O</smiles>

Fig. 7 Structures of catechins and epicatechins 139-154 from Actinidia plants 
Table 6 Information of catechins and epicatechins from Actinidia plants

\begin{tabular}{|c|c|c|c|c|}
\hline No. & Compound name & Species Refs. & Part & Bioactivity Refs. \\
\hline 139 & $(+)$-Catechin & A. arguta $[41]$ & Roots & $\begin{array}{l}\text { Anti-DPPH radical and inhibiting nitric oxide } \\
\text { (in vitro) [57] } \\
\text { Anti-bacteria } \\
\text { (in vitro) [93] }\end{array}$ \\
\hline 140 & (-)-Epi-catechin & A. arguta $[41]$ & Roots & $\begin{array}{l}\text { Anti-DPPH radical and inhibiting nitric oxide } \\
\text { (in vitro) [57] }\end{array}$ \\
\hline 141 & $(+)$-Afzelechin & A. chinensis Planch [94] & Roots & \\
\hline 142 & $(-)$-Epi-afzelechin & A. chinensis Planch [94] & Roots & \\
\hline 143 & $(+)$-Catechin $(4 \alpha \rightarrow 8)(+)$-catechin & A. chinensis Planch [94] & Roots & \\
\hline 144 & $(-)$-Epi-catechin $(4 \beta \rightarrow 8)(-)$-epi-catechin & A. chinensis Planch [94] & Roots & \\
\hline 145 & $(+)$-Afzelechin $(4 \alpha \rightarrow 8)(+)$-afzelechin & A. chinensis Planch [94] & Roots & \\
\hline 146 & $(-)$-Epi-afzelechin $(4 \beta \rightarrow 8)(-)$-epi-afzelechin & A. chinensis Planch [94] & Roots & \\
\hline 147 & Gallocatechin & A. deliciosa [89] & Peels & $\begin{array}{l}\text { Radical scavenging and antioxidant } \\
\text { (in vitro) [89] }\end{array}$ \\
\hline 148 & 6-(2-Pyrrolidinone-5-yl)-(-)-epicatechin & A. arguta [92] & Roots & $\begin{array}{l}\text { Against advanced glycation-end } \\
\text { (in vitro) [92] }\end{array}$ \\
\hline 149 & 8-(2-Pyrrolidinone-5-yl)-(-)-epicatechin & A. arguta [92] & Roots & $\begin{array}{l}\text { Against advanced glycation-end } \\
\text { (in vitro) [92] }\end{array}$ \\
\hline 150 & Proanthocyanidin B-4 & A. arguta [92] & Roots & $\begin{array}{l}\text { Against advanced glycation-end } \\
\text { (in vitro) [92] }\end{array}$ \\
\hline 151 & (-)-Epi-Catechin-5-O- $\beta$-D-glucopyranoside & A. arguta [92] & Roots & $\begin{array}{l}\text { Against advanced glycation-end } \\
\text { (in vitro) [92] }\end{array}$ \\
\hline 152 & Benzylthio-(-)-epicatechol & A. chinensis [95] & Vegetative parts & \\
\hline 153 & 4'-Benzylthioprocyanidol B2 & A. chinensis [95] & Vegetative parts & \\
\hline 154 & Procyanidol C1 & A. chinensis [95] & Vegetative parts & \\
\hline
\end{tabular}

\subsubsection{Emodins}

A total of nine emodin derivatives were obtained (211-219, Fig. 9, Table 8). Three emodin constituents were isolated from EtOAc fraction of the roots of A. deliciosa for the first time, and their structures were identified to be aloe-emodin 211, 11-O-acetyl-aloe-emodin 212, and aloe-emodin 11-O- $\alpha$-L-rhamno -pyranoside 213 [125]. Compound 211 exhibited intriguing biological activities including inflammatory, antifungal, and anticancer activity [126-128]. Lipoxygenases (LOXs) are potential treatment targets in a variety of inflammatory conditions, enzyme kinetics showed that aloe emodin inhibited lipoxygenase competitively with an $\mathrm{IC}_{50}$ of $29.49 \mu \mathrm{M}$ [126]. Compound 215 was reported to possess wide biological activities including anti-inflammatory, neuroprotection, anti-cardiovascular and $\alpha$-glucosidase inhibitory activity [129-132]. It exhibited potent inhibition of $\alpha$-glucosidase with an $\mathrm{IC}_{50}$ value of $19 \pm 1 \mu \mathrm{M}$ and lower cytotoxicity to the Caco-2 cell line [132].

\subsubsection{Phenylpropionic Acids}

A total of 38 phenylpropionic acid derivatives have been identified from kiwifruit plants (220-257. Fig. 10, Table 9), while most of them were glycosides or quinic acid derivatives. Phytochemical examination of the fruits of A. arguta led to the isolation of two organic acids including caffeic acid 220 and caffeoyl- $\beta$-D-glucopyranoside 221, which were tested for their nitric oxide production inhibitory activity in LPS-stimulated RAW 264.7 cells and DPPH radical scavenging activities. Compared with positive control (L-NMMA), they were potently reduced nitric oxide productions and showed anti-oxidative activities [135]. Nine succinic acid derivatives (228-236), eleven quinic acid (245-255) derivatives and two shikimic acid derivatives (256 and 257) were isolated from the fruits of A. arguta. The NF- $\kappa \mathrm{B}$ transcriptional inhibitory activity of the compounds was evaluated using RAW 264.7 macrophages cells induced by lipopolysaccharide. Among the groups of 
<smiles>COc1ccc(-c2coc3cc(O)ccc3c2=O)cc1</smiles><smiles></smiles><smiles>O=c1cc(-c2ccc(O)cc2)oc2cc(O)c([C@@H]3O[C@H](CO)[C@@H](O)[C@H](O)[C@H]3O)c(O)c12</smiles><smiles>O=c1cc(-c2ccc(O)cc2)oc2c([C@@H]3OC[C@@H](O)[C@H](O)[C@H]3O)c(O)c([C@@H]3O[C@H](CO)[C@@H](O)[C@H](O)[C@H]3O)c(O)c12</smiles><smiles>O=c1cc(-c2ccc(O)cc2)oc2c(O[C@@H]3O[C@H](CO)[C@@H](O)[C@H](O)[C@H]3O)c(O)c(O[C@@H]3O[C@H](CO)[C@@H](O)[C@H](O)[C@H]3O)c(O)c12</smiles><smiles>O=c1c(O[C@@H]2O[C@H](CO)[C@@H](O)[C@H](O)[C@H]2O)c(-c2ccc(O)cc2)oc2cc(O)cc(O)c12</smiles><smiles>C[C@H]1O[C@H](Oc2c(-c3ccc(O)cc3)oc3cc(O)cc(O)c3c2=O)[C@H](O)[C@@H](O)[C@@H]1O</smiles><smiles></smiles><smiles>O=c1c(O[C@@H]2O[C@H](CO)[C@@H](O)[C@H](O)[C@H]2O)c(-c2ccc(O)cc2)oc2cc(O)cc(O)c12</smiles><smiles>C[C@H]1O[C@@H](Oc2cc(O)c3c(=O)c(O)c(-c4ccc(O)cc4)oc3c2)[C@H](O)[C@@H](O)[C@@H]1O</smiles><smiles>C[C@H]1O[C@H](OC[C@H]2O[C@H](Oc3c(-c4ccc(O)cc4)oc4cc(O)cc(O)c4c3=O)[C@H](O)[C@H](O)[C@@H]2O)[C@H](O)[C@@H](O)[C@@H]1O</smiles>

Fig. 8 Structures of flavones, isoflavones, and flavonols 155-202 from Actinidia plants 


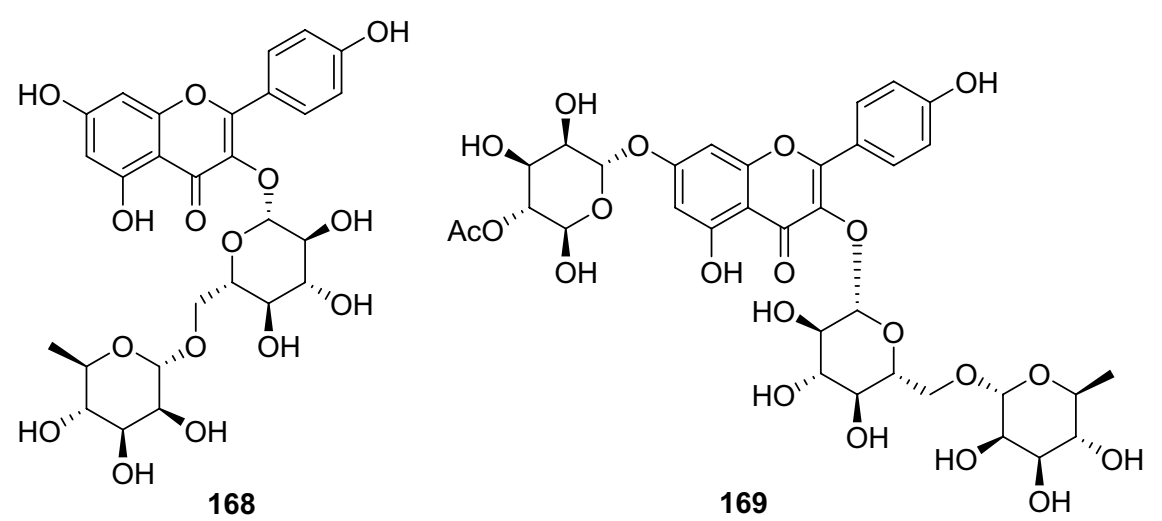

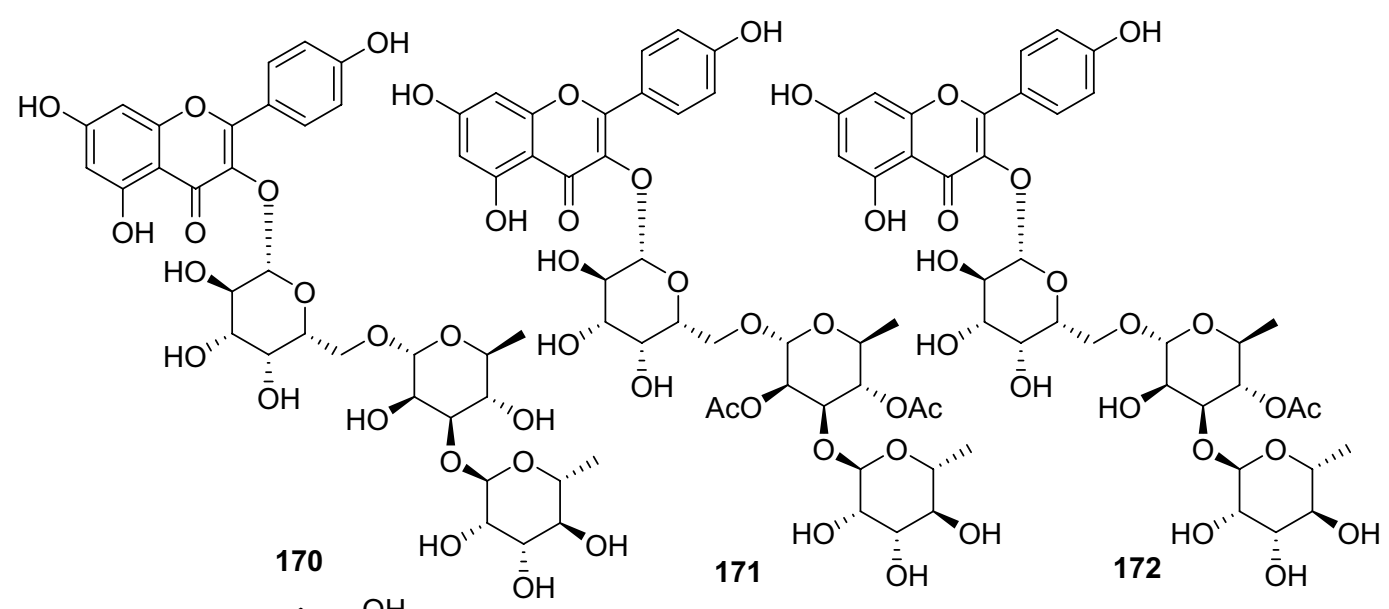

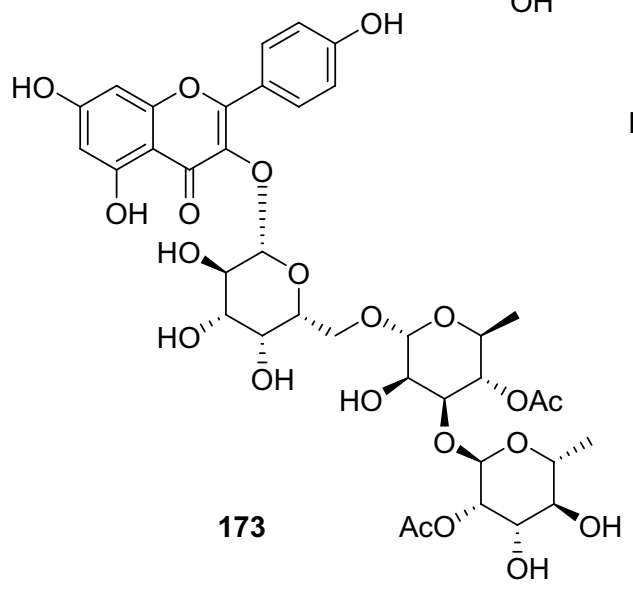

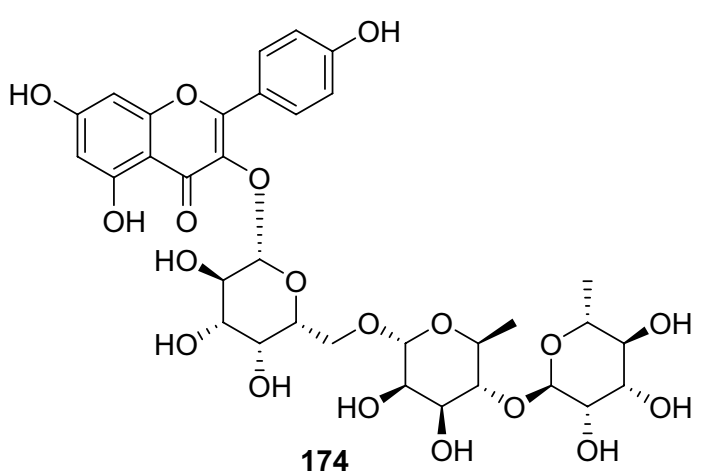

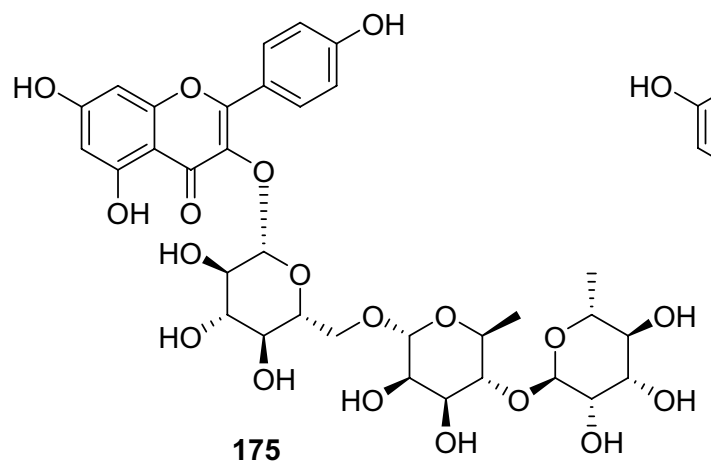

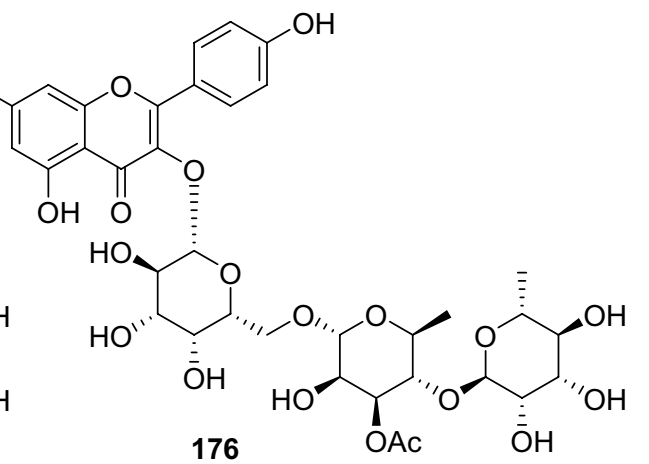

Fig. 8 (continued) 
<smiles>C[C@H]1O[C@H](OC[C@H]2O[C@H](Oc3c(-c4ccc(O)cc4)oc4cc(O[C@@H]5O[C@H](C)[C@@H](O)[C@H](O)[C@H]5O)cc(O)c4c3=O)[C@H](O)[C@H](O)[C@@H]2O)[C@H](O)[C@@H](O)[C@@H]1O</smiles>

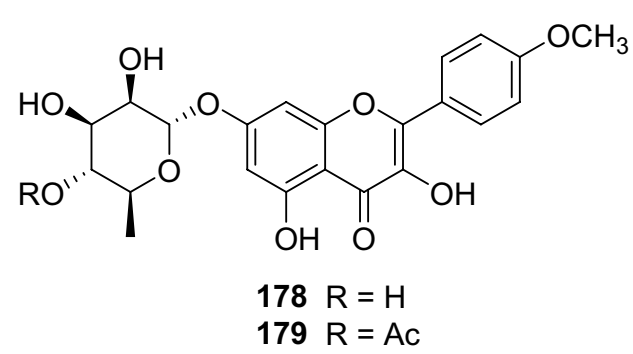

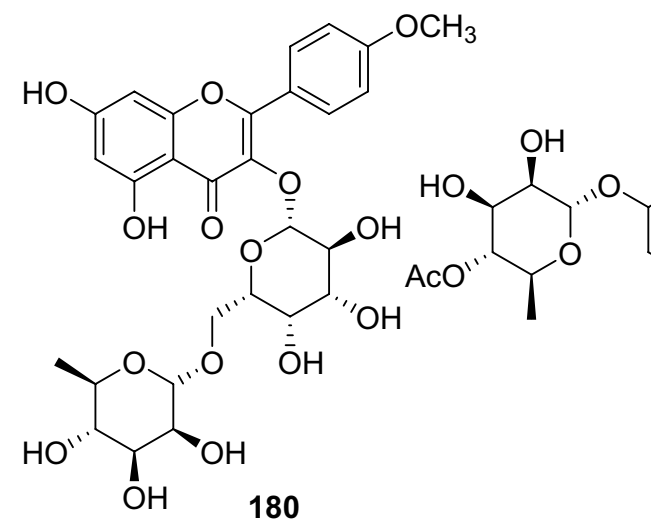<smiles></smiles><smiles>COc1ccc(-c2oc3cc(O[C@@H]4O[C@H](C)[C@@H](O)[C@H](O)[C@H]4O)cc(O)c3c(=O)c2O[C@H]2O[C@H](CO[C@@H]3O[C@H](C)[C@@H](O)[C@H](O)[C@H]3O)[C@@H](O)[C@H](O)[C@H]2O)cc1</smiles><smiles>O=c1c(O)c(-c2ccc(O)c(O)c2)oc2cc(O)cc(O)c12</smiles>

185<smiles>O=c1c(O[C@@H]2O[C@H](CO)[C@@H](O)[C@H](O)[C@H]2O)c(-c2ccc(O)c(O)c2)oc2cc(O)cc(O)c12</smiles><smiles>C[C@@H]1O[C@H](Oc2c(-c3ccc(O)c(O)c3)oc3cc(O)cc(O)c3c2=O)[C@H](O)[C@H](O)[C@@H]1O</smiles>

Fig. 8 (continued) 


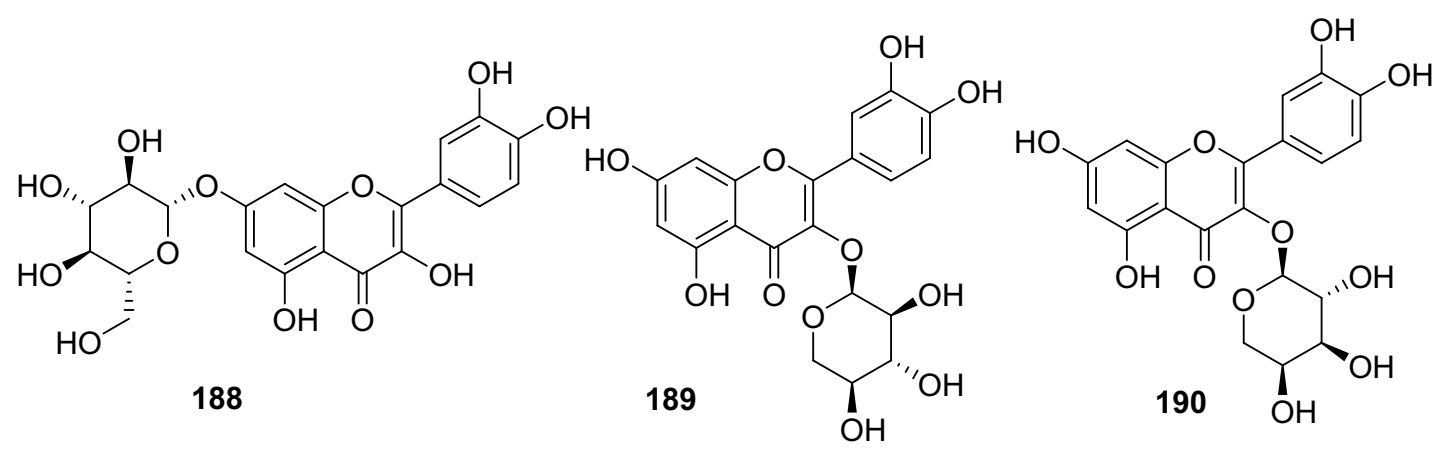

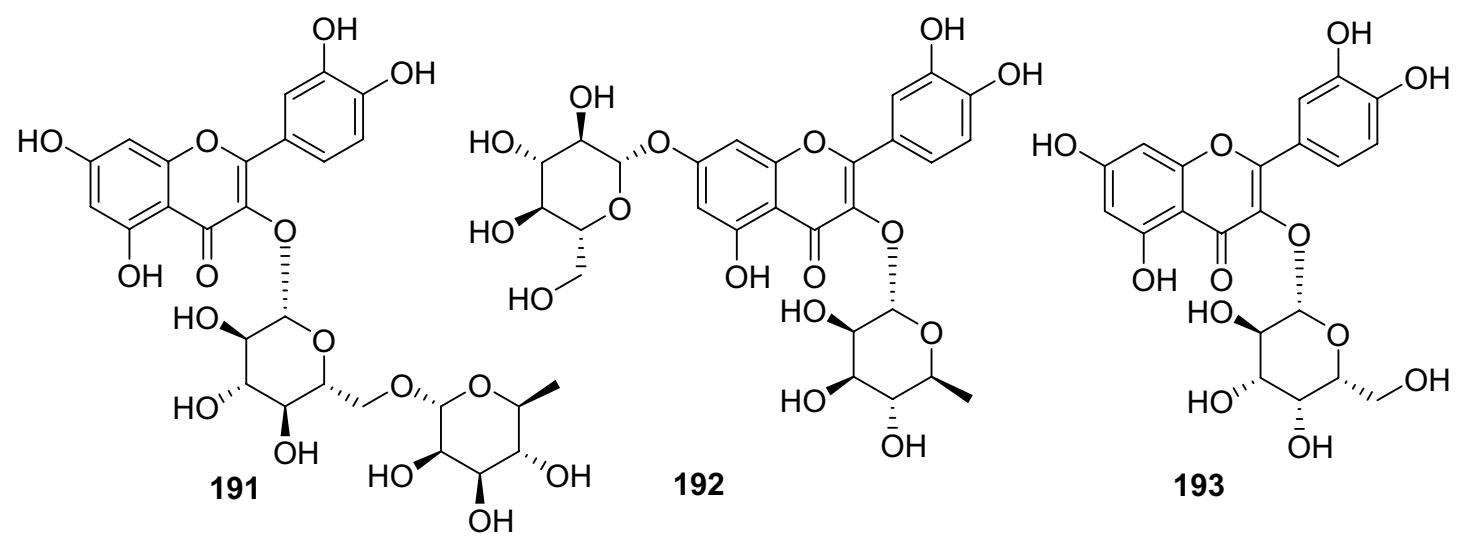<smiles>O=c1c(O[C@@H]2O[C@H](C[C@@H](O)CO)[C@@H](O)[C@H]2O)c(-c2ccc(O)c(O)c2)oc2cc(O)cc(O)c12</smiles><smiles>C[C@H]1O[C@H](CO[C@H]2O[C@H](Oc3c(-c4ccc(O)c(O)c4)oc4cc(O)cc(O)c4c3=O)[C@H](O)[C@H](O)[C@H]2O)[C@H](O)[C@@H](O)[C@H]1O</smiles><smiles>COc1cc(-c2oc3cc(O)cc(O)c3c(=O)c2O[C@@H]2O[C@H](CO)[C@@H](O)[C@H](O)[C@H]2O)ccc1O</smiles><smiles>C[C@H]1O[C@H](O[C@H]2[C@@H](C)O[C@H](OC[C@H]3O[C@H](Oc4c(-c5ccc(O)c(O)c5)oc5cc(O)cc(O)c5c4=O)[C@@H](O)[C@H](O)[C@H]3O)[C@H](O)[C@H]2O)[C@H](O)[C@@H](O)[C@@H]1O</smiles>

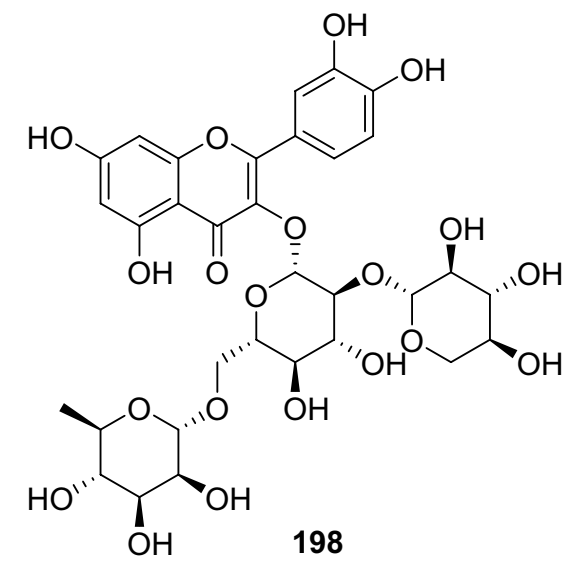

Fig. 8 (continued) 
<smiles>O=c1c(O[C@@H]2O[C@H](CO)[C@@H](O)[C@H](O)[C@H]2O[C@H]2OC[C@@H](O)[C@H](O)[C@H]2O)c(-c2ccc(O)c(O)c2)oc2cc(O)cc(O)c12</smiles><smiles>COc1ccc(C2CC(=O)c3c(O)cc(O[C@@H]4O[C@H](CO[C@H]5O[C@@H](C)[C@@H](O)[C@H](O)[C@H]5O)[C@@H](O)[C@H](O)[C@H]4O)cc3O2)cc1O</smiles><smiles>COc1ccc(-c2oc3cc(O[C@@H]4OC(C)[C@@H](OC(C)=O)[C@H](O)[C@H]4O)cc(O)c3c(=O)c2O[C@@H]2O[C@H](CO)[C@@H](O)[C@H](O)[C@H]2O)cc1O</smiles>

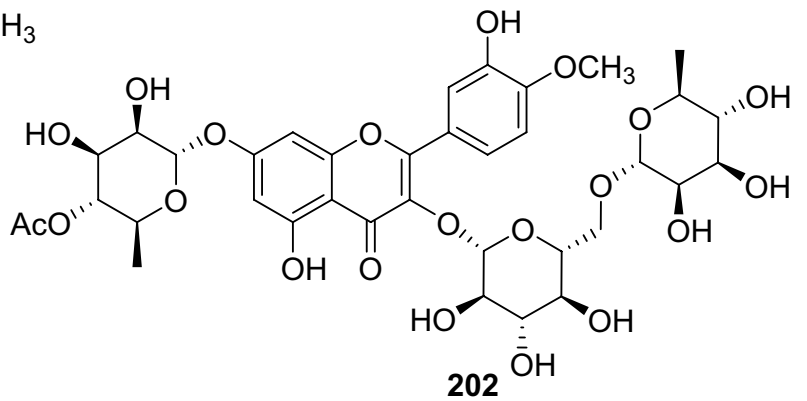

Fig. 8 (continued)

different organic acid derivatives, the quinic acid derivatives inhibited NF- $\kappa \mathrm{B}$ transcriptional activity with an $\mathrm{IC}_{50}$ value of $4.0 \mu \mathrm{M}$ [136].

\subsubsection{Coumarins}

Coumarins are rarely identified from kiwifruit plants, and only eleven members have been reported (258-267, Fig. 11, Table 10). Umbelliferone $\mathbf{2 5 8}$ was obtained from the leaves of A. polygama (Sieb. et Zucc.) Miq [109]. A number of studies demonstrate the pharmacological properties of $\mathbf{2 5 8}$ including antitumor, anti-inflammatory, antioxidant, antidiabetic, and immunomodulatory activities [143-149]. It showed cytotoxicity against MCF-7 and MDA-MB-231 cell lines with $\mathrm{IC}_{50}$ values of 15.56 and $10.31 \mu \mathrm{M}$, respectively [148]. Phytochemical examination of the fruits of A. arguta led to the isolation of esculetin 259 [135]. Two coumarins were isolated from the roots of A. deliciosa and identified as fraxetin 260 and isoscopoletin 261 [150]. Compound 260 showed potent inhibition against lipopolysaccharide (LPS)-induced nitric oxide (NO) generation with an $\mathrm{IC}_{50}$ value of $10.11 \pm 0.47 \mu \mathrm{M}$ [151]. Esculin 263 and fraxin 264 were characterized from the stems and fruits of A. deliciosa (kiwifruit) and A. chinensis [152]. Compound 264 showed inhibitory activity towards $\mathrm{HepG} 2$ with an $\mathrm{IC}_{50}$ value of $14.71 \mu \mathrm{M}$ [153].

\subsubsection{Lignans}

Lignans also had a narrow distribution in kiwi plants, only six members have been identified from Actinidia plants (269-274, Fig. 12, Table 11). (+)-Pinoresinol 271, (+)-medioresinol 272, and (-)-syringaresinol 273 were partitioned from the fraction of the roots of A. arguta [53]. Compound $\mathbf{2 7 1}$ is a biologically active lignan and widely found in many dietary plants. It was reported to possess antifungal, anti-inflammatory, antioxidant, hypoglycemic, and antitumor activities [158-162]. A study on this compound suggested that $\mathbf{2 7 1}$ displayed significant inhibition of fMLP/CB-induced superoxide anion generation and elastase release, with an $\mathrm{IC}_{50}$ value of $1.3 \pm 0.2 \mu \mathrm{g} / \mathrm{mL}$ [159]. The $50 \%$ ethanol extract of $A$. arguta showed strong inhibitory effect on $\alpha$-glucosidase (32.6\%), while a bio-guided isolation on the extract gave a bioactive compound pinoresinol diglucoside 274 [138]. 
Table 7 Information of flavones, isoflavones, and flavonols from Actinidia plants

\begin{tabular}{|c|c|c|c|c|}
\hline No. & Compound name & Species Refs. & Part & Bioactivity Refs. \\
\hline 155 & 7,4'-Dihydroxyflavone & A. chinensis Planch [105] & Roots & $\begin{array}{l}\text { Anti-inflammatory } \\
\text { (in vitro) [106] }\end{array}$ \\
\hline 156 & Formononetin & A. chinensis Planch [105] & Roots & \\
\hline 157 & Kaempferol & A. deliciosa [107] & Leaves & $\begin{array}{l}\text { Antiproliferation human tumor cell } \\
\text { (in vitro) [96] } \\
\text { Antioxidant and anti-inflammatory } \\
\text { (in vitro) [97] } \\
\text { Anticancer (in vitro) [98] } \\
\text { Neuroprotection } \\
\text { (in vitro) [99] }\end{array}$ \\
\hline 158 & 6-C-Glucose-5,7,3',4'-tetrahydroxy flavone & A. arguta $[108]$ & Roots & \\
\hline 159 & 6-C-Glucose-5,7,4'-trihydroxy flavone & A. arguta $[108]$ & Roots & \\
\hline 160 & 6-C-Glycopyranosyl-8-C-xyloeyl apigenin & A. polygama [109] & Leaves & \\
\hline 161 & Vicenin-I & A. polygama $[110]$ & Aerial Parts & \\
\hline 162 & Kaempferol 3-O-glucoside & A. deliciosa [107] & Leaves & \\
\hline 163 & Kaempferol 3-O-rhamnoside & A. deliciosa [107] & Leaves & \\
\hline 164 & Kaempferol 7-O-glucoside & A. deliciosa [107] & Leaves & \\
\hline 165 & Kaempferol 3-O- $\beta$-D-galactopyranoside & A. polygama [109] & Leaves & \\
\hline 166 & Kaempferol-7-O- $\beta$-L-rhamnoside & A. kolomikta [102] & Leaves & \\
\hline 167 & $\begin{array}{l}\text { Kaempnferol-3-O- } \alpha \text {-L- } \\
\text { rhamnopyranosyl- }(1 \rightarrow 6)-\beta \text {-D- } \\
\text { galactopyranoside }\end{array}$ & A. polygama [109] & Leaves & \\
\hline 168 & Kaempferol 3-O-rutinoside & A. deliciosa [107] & Leaves & \\
\hline 169 & $\begin{array}{l}\text { Kaempferol-7-O-(4"-O-acetylrhamnosyl)-3-O- } \\
\text { rutinoside }\end{array}$ & A. kolomikta [111] & Leaves & \\
\hline 170 & $\begin{array}{l}\text { Kaempferol-3-O- } \alpha \text {-L-rhamnopyranosyl- } \\
(1 \rightarrow 3)-\alpha \text {-L-rhamnopyranosyl- }(1 \rightarrow 6)-\beta \text {-D- } \\
\text { galactopyranoside }\end{array}$ & A. polygama [109] & Leaves & \\
\hline 171 & $\begin{array}{l}\text { Kaempferol 3-O- } \alpha \text {-L-rhamnopyranosyl- }(1 \rightarrow 3) \\
(2,4 \text {-di-O-acetyl- } \alpha \text {-L-rhamnopyranosyl })(1 \rightarrow 6) \\
\beta \text {-D-galactopyranoside }\end{array}$ & A. valvata Dunn [100] & Leaves & $\begin{array}{l}\text { Anti-free radical } \\
\text { (in vitro) [100] }\end{array}$ \\
\hline 172 & $\begin{array}{l}\text { Kaempferol 3-O- } \alpha \text {-L-rhamnopyranosyl }(1 \rightarrow 3) \\
\text { (4-O-acetyl- } \alpha \text {-L-rhamnopyranosyl) }(1 \rightarrow 6) \\
\beta \text {-D-galactopyranoside }\end{array}$ & A. valvata Dunn [100] & Leaves & $\begin{array}{l}\text { Anti-free radical } \\
\text { (in vitro) }[100]\end{array}$ \\
\hline 173 & $\begin{array}{l}\text { Kaempferol 3-O-[ } \alpha \text {-L-rhamnopyranosyl- }(1 \rightarrow 3) \text { - } \\
\text { (4-O-acetyl)-O- } \alpha \text {-L-rhamnopyranosyl- }(1 \rightarrow 6) \text { - } \\
\text { O-acetyl)-O- } \beta \text {-D-galactopyranoside }]\end{array}$ & A. polygama $[110]$ & Aerial parts & \\
\hline 174 & $\begin{array}{l}\text { Kaempferol 3-O- }[\alpha \text {-rhamnopyranosyl- }(1 \rightarrow 4)- \\
\text { rhamnopyranosyl- }(1 \rightarrow 6)-\beta \text {-galactopyranoside }]\end{array}$ & A. deliciosa [112] & Leaves & \\
\hline 175 & $\begin{array}{l}\text { Kaempferol 3-O-[ } \alpha \text {-rhamnopyranosyl- }(1 \rightarrow 4) \text { - } \\
\text { rhamnopyranosyl- }(1 \rightarrow 6) \text { - } \beta \text {-glucopyranoside] }\end{array}$ & A. deliciosa [112] & Leaves & \\
\hline 176 & $\begin{array}{l}\text { Kaempferol 3-O-[ } \alpha \text { - } \\
\text { rhamnopyranosyl- }(1 \rightarrow 4)-3^{\prime \prime \prime} \\
\text {-O-acetyl- } \alpha \text { - } \\
\text { rhamnopyranosyl- }(1 \rightarrow 6) \text { - } \beta \text {-galactop- } \\
\text { yranoside] }\end{array}$ & A. deliciosa [112] & Leaves & \\
\hline 177 & Kaempferol-7-O- $\alpha$-L-rhamnosyl-3-O-rutinoside & A. kolomikta [102] & Leaves & \\
\hline 178 & Kaempferide-7-O-rhamnoside & A. kolomikta $[101]$ & Leaves & \\
\hline 179 & Kaempferide-7-O-(4"-O-acetyl)- $\alpha$-L-rhamnoside & A. kolomikta [102] & Leaves & $\begin{array}{l}\text { Against AAPH-induced hemolysis } \\
\text { (in vitro) [102] }\end{array}$ \\
\hline 180 & Kaempferide-3-O-rutinoside & A. kolomikta [113] & Leaves & $\begin{array}{l}\text { Anti-free radical } \\
\text { (in vitro) [113] }\end{array}$ \\
\hline 181 & $\begin{array}{l}\text { Kaempferide-7-O-(4"-O-acetyl-rhamnosyl)-3-O- } \\
\text { glucoside }\end{array}$ & A. kolomikta [113] & Leaves & $\begin{array}{l}\text { Anti-free radical } \\
\text { (in vitro) [113] }\end{array}$ \\
\hline
\end{tabular}


Table 7 (continued)

\begin{tabular}{|c|c|c|c|c|}
\hline No. & Compound name & Species Refs. & Part & Bioactivity Refs. \\
\hline 182 & $\begin{array}{l}\text { Kaempferide-7-O-(4"-O-acetyl-rhamnosyl)-3-O- } \\
\text { rutinoside }\end{array}$ & A. kolomikta [113] & Leaves & $\begin{array}{l}\text { Anti-free radical } \\
\text { (in vitro) [113] } \\
\text { Reducing myocardial infarction (in vivo) [104] }\end{array}$ \\
\hline 183 & Kaempferide-7-O-rhamnosyl-3-O-rutinoside & A. kolomikta [101] & Leaves & \\
\hline 184 & $\begin{array}{l}\text { Kaempferide-7-O-(3"-O-acetylrhamnosyl)-3-O- } \\
\text { rutinoside }\end{array}$ & A. kolomikta $[111]$ & Leaves & \\
\hline 185 & Quercetin & A. deliciosa [107] & Leaves & \\
\hline 186 & Quercetin-3-O- $\beta$-D-glucoside & A. deliciosa [107] & Leaves & \\
\hline 187 & Quercetin 3-O-rhamnoside & A. deliciosa [107] & Leaves & \\
\hline 188 & Quercetin 7-O-glucoside & A. deliciosa [107] & Leaves & \\
\hline 189 & Quercetin 3-O-xyloside & A. deliciosa [107] & Leaves & \\
\hline 190 & Quercetin 3-O-arabinoside & A. deliciosa [107] & Leaves & \\
\hline 191 & Quercetin 3-O-rutinoside & A. deliciosa [107] & Leaves & \\
\hline 192 & Quercetin 3-O-rhamnoside 7-O-glucoside & A. deliciosa [107] & Leaves & \\
\hline 193 & Quercetin 3-O- $\beta$-D-galactoside & A. polygama $[110]$ & Aerial parts & $\begin{array}{l}\text { Anti-DPPH radical and nitric oxide production } \\
\text { (in vitro) [110] }\end{array}$ \\
\hline 194 & $\begin{array}{l}\text { Quercetin 3-O- } \beta \text {-D-glucofuranoside (isoquerci- } \\
\text { trin) }\end{array}$ & A. chinensis Planch [114] & & \\
\hline 195 & $\begin{array}{l}\text { Quercetin3-O- } \alpha \text {-L-rhamnopyranosyl- }(1 \rightarrow 6)-\beta \text {-D- } \\
\text { galactopyranoside }\end{array}$ & A. arguta [108] & Roots & \\
\hline 196 & Isorhamnetin-3-O- $\beta$-D-glucoside & A. kolomikta [102] & Leaves & \\
\hline 197 & $\begin{array}{l}\text { Quercetin3-O- }[\alpha \text {-rhamnopyranosyl- }(1 \rightarrow 4) \\
\text {-rhamnopyranosyl- }(1 \rightarrow 6) \text { - } \beta \text {-galactopyranoside }\end{array}$ & A. deliciosa [112] & Leaves & \\
\hline 198 & $\begin{array}{l}\text { Quercetin 3-O- } \beta \text {-D-[2 } 2^{\mathrm{G}} \text {-O- } \beta \text {-D-xylopyranosyl- } 6^{\mathrm{G}}- \\
\text { O- } \alpha \text {-L-rhamnopyranosyl] glucopyranoside }\end{array}$ & A. arguta [115] & Leaves & \\
\hline 199 & $\begin{array}{l}\text { Quercetin 3-O- } \beta \text {-D-xylopyranosyl- }(1 \rightarrow 2)-\mathrm{O}-\beta \text { - } \\
\text { D-glucopyranoside }\end{array}$ & A. arguta [115] & Leaves & \\
\hline 200 & $\begin{array}{l}\text { 7-O-[ } \beta \text {-D-Pyranrhamnose- }(1 \rightarrow 6)-\beta \text {-D-pyran } \\
\text { glucose }]-5,3^{\prime} \text {-dihydroxy-4'-methoxy two dihy- } \\
\text { drogen flavone }\end{array}$ & A. arguta [108] & Roots & \\
\hline 201 & $\begin{array}{l}\text { 4'-Methoxyl-quercetin-7-(4"-O- } \\
\text { acetylrhamnosyl)-3-O- } \beta \text {-D-glucopyranoside }\end{array}$ & A. kolomikta [111] & Leaves & \\
\hline 202 & $\begin{array}{l}\text { 4'-Methoxyl-quercetin-7-(4"-O- } \\
\text { acetylrhamnosyl)-3-O-rutinoside }\end{array}$ & A. kolomikta [111] & Leaves & \\
\hline
\end{tabular}

\subsubsection{Simple Phenols}

Simple benzene derivatives including glycosides and isoprenylated benzene products from Actinidia plants were collected (275-298, Fig. 13, Table 12). Phytochemical examination of the fruits of $A$. arguta led to the isolation of protocatechuic acid 279 [135]. It showed anti-inflammatory [163], antioxidant [163], neuroprotective [164], and antiproliferative activities [165]. Protocatechuic acid exhibited significant $(\mathrm{p}<0.05)$ anti-inflammatory $(83 \%$ and $88 \%$ inhibition for egg-albumin induced and xylene induced oedema, respectively), analgesic (56\% inhibition and $22 \mathrm{~s}$ of pain suppression for acetic acid-induced and hot plate-induced pain, respectively), and antioxidant effects (97\% inhibition and absorbance of 2.516 at $100 \mu \mathrm{g} / \mathrm{mL}$ for DPPH and FRAP assay, respectively) in the models [166]. Extraction of leaf tissue from the golden-fleshed kiwifruit cultivar A. chinensis "Hort16A" expressing genotype-resistance against the fungus Botrytis cinerea, a new phenolic compound, 3,5-dihydroxy-2-(methoxycarbonylmethyl)phenyl 3,4-dihydroxybenzoate $\mathbf{2 7 8}$ was therefore obtained [167].

Four novel skeleton phenolic compounds planchols A-D (291-294) were isolated from the roots of A. chinensis Planch. Their structures were elucidated by spectroscopic analysis and chemical evidence. The structure of $\mathbf{2 9 1}$ was further confirmed by the single-crystal X-ray diffraction. Moreover, it was found that $\mathbf{2 9 1}$ and $\mathbf{2 9 2}$ showed remarkable cytotoxic activity against $\mathrm{P}-388$ with $\mathrm{IC}_{50}$ of 2.50 and 
Fig. 9 Structures of xanthones, isoflavones, and flavonols 203-219 from Actinidia plants<smiles>C=C(/C=C/OC(/C=C\C)=C/C)OC</smiles>

$\mathrm{HO}$,<smiles>C=C/C(O[C@@H]1O[C@H](CO[C@H]2OCC[C@@H](O)[C@H]2O)[C@@H](O)[C@H](O)[C@H]1O)=C(\C)C(=O)C(=C)/C(O)=C(\C)OC</smiles>

204<smiles>O=C(O)C[C@H]1O[C@@H](Oc2cc3c(O)cc(O)cc3[o+]c2-c2cc(O)c(O)c(O)c2)[C@H](O)[C@H](O)[C@H]1O</smiles>

205<smiles></smiles><smiles>OC[C@H]1O[C@@H](Oc2cc3c(O)cc(O)cc3[o+]c2-c2cc(O)c(O)c(O)c2)[C@H](O[C@@H]2OC[C@@H](O)[C@H](O)[C@H]2O)[C@H](O)[C@H]1O</smiles><smiles>OC[C@H]1O[C@@H](Oc2cc3c(O)cc(O)cc3[o+]c2-c2ccc(O)c(O)c2)[C@H](O[C@@H]2OC[C@@H](O)[C@H](O)[C@H]2O)[C@H](O)[C@H]1O</smiles>
209<smiles>[R12]Cc1cc(O)c2c(c1)C(=O)c1cccc(O)c1C2=O</smiles><smiles>[Z13]Oc1cc(C[O+][C@@H]2O[C@H](C)[C@@H](O)[C@H](O)[C@H]2O)cc2c1C(=O)c1c(O)cccc1C2=O</smiles><smiles>[Z14]C=C1c2c(O)cc(C)cc2C(=O)c2cc(OC)cc(O)c21</smiles><smiles>[Z15]Cc1c(O)cc(O)cc1C(=O)c1cc(C)cc2c1C(=O)c1cc(O)cc(O)c1-2</smiles><smiles>[Z20]Cc1cc(C)cc(O)c1C(=O)c1cc(O)cc(OC)c1</smiles>

$\mathrm{HO} \backslash$<smiles>O=C1c2cc(CO)cc(O)c2C(=O)c2cc(O)cc(O)c21</smiles>

217<smiles>O=C(O)c1cc(O)c2c(c1)C(=O)c1cc(O)cc(O)c1C2=O</smiles>

218<smiles></smiles> 
Table 8 Information of xanthones, anthocyanins, and emodins from Actinidia plants

\begin{tabular}{|c|c|c|c|c|}
\hline No. & Compound name & Species Refs. & Part & Bioactivity Refs. \\
\hline 203 & $2-\beta$-D-Glu-1,3,7-trihydrogen xanthone & A. arguta $[108]$ & Roots & $\begin{array}{l}\text { Inhibiting } \alpha \text {-Glycosidase } \\
\text { (in vitro) [116] } \\
\text { NO inhibitory (in vitro) [117] } \\
\text { NF- } \kappa \text { B Inhibition and PPAR Activation } \\
\text { (in vitro) [118] }\end{array}$ \\
\hline 204 & $\begin{array}{l}\text { 7-O-[ } \beta \text {-D-Xylose- }(1 \rightarrow 6)-\beta \text {-D- } \\
\text { glucopyranoside]-1,8-dihydroxy-3- } \\
\text { methoxy xanthone }\end{array}$ & A. arguta [108] & Roots & \\
\hline 205 & $\begin{array}{l}\text { 1-O-[ } \beta \text {-D-Xylose- }(1 \rightarrow 6)-\beta \text {-D- } \\
\text { glucopyranoside] }-8 \text {-hydroxy-3,7-dimeth- } \\
\text { oxy xanthone }\end{array}$ & A. arguta $[108]$ & Roots & \\
\hline 206 & Delphinidin 3-galactoside & A. deliciosa [119] & Fruits & Antioxidation (in vitro) [135] \\
\hline 207 & Cyanidin 3-galactoside & A. deliciosa [119] & Fruits & \\
\hline 208 & Cyanidin 3-glucoside & A. deliciosa [119] & Fruits & $\begin{array}{l}\text { Anti-inflammatory } \\
\text { (in vitro) }[120,121] \text { Neuroprotective (in vivo) [122] } \\
\text { Anti-cancer (in vitro) [123] } \\
\text { Antioxidation (in vivo) [124] }\end{array}$ \\
\hline 209 & Delphinidin 3-[2-(xylosyl)galactoside] & A. deliciosa [119] & Fruits & \\
\hline 210 & Cyanidin 3-[2-(xylosyl)galactoside] & A. deliciosa [119] & Fruits & \\
\hline 211 & Aloe-emodin & A. deliciosa [125] & Roots & $\begin{array}{l}\text { Anti-inflammatory } \\
\text { (in vitro) [126] } \\
\text { Antifungal (in vitro) [127] } \\
\text { Anticancer (in vitro) [128] }\end{array}$ \\
\hline 212 & 11-O-Acetyl-aloe-emodin & A. deliciosa [125] & Roots & \\
\hline 213 & Aloe-emodin 11-O- $\alpha$-L-rhamnopyranoside & A. deliciosa [125] & Roots & \\
\hline 214 & Physcion (emodin-6-Me ether) & A. chinensis Planch [133] & Roots & \\
\hline 215 & Emodin (frangala-emodin) & A. chinensis Planch [133] & Roots & $\begin{array}{l}\text { Anti-inflammatory (in vivo and in vitro) [129] } \\
\text { Neuroprotection (in vitro) [130] } \\
\text { Anti-cardiovascular } \\
\text { (in vitro) [131] } \\
\text { Inhibiting } \alpha \text {-Glucosidase (in vitro) [132] }\end{array}$ \\
\hline 216 & Questin (emodin-8-Me ether) & A. chinensis Planch [133] & Roots & $\begin{array}{l}\text { Hepatoprotection } \\
\text { (in vitro) [134] }\end{array}$ \\
\hline 217 & Citreorosein ( $\omega$-hydroxyemodin) & A. chinensis Planch [133] & Roots & \\
\hline 218 & Emodic acid & A. chinensis Planch [133] & Roots & \\
\hline 219 & Emodin-8- $\beta$-D-glucoside & A. chinensis Planch [133] & Roots & \\
\hline
\end{tabular}

$3.85 \mu \mathrm{M}$, respectively, and against $\mathrm{A}-549$ with $\mathrm{IC}_{50}$ of 1.42 and $2.88 \mu \mathrm{M}$, respectively [94].

\subsection{Miscellaneous}

Three alkaloids (299-301), eleven fatty acids and derivatives (302-312), and other thirteen small molecules (313-325) were obtained from Actinidia plants (Fig. 14, Table 13). Actinidine 299 and boschniakine 300 were isolated from the leaves and galls of A. polygama and also isolated from $A$. arguta which might be converted from iridoids [174, 175]. A bioassay-guided fractionation of the fruits of A. polygama led to the separation and identification of a polyunsaturated fatty acid, $\alpha$-linolenic acid (ALA) $\mathbf{3 0 5}$ [176]. This compound was found to possess a broad biological properties including anti-inflammatory [177], anti-tumor [178], anti-hyperlipidemic [179], anti-diabetic [180], and anti-fungal [181] activities. By a bio-guided fractionation, a ceramide namely actinidiamide $\mathbf{3 1 2}$ was identified as an 
<smiles>[R2][R20]([R2])=C/C=C/c1ccc(O)c([R])c1</smiles><smiles>[R6]c1ccc(/C=C/C(=O)O)cc1O[C@@H]1O[C@H](CO)[C@@H](O)[C@H](O)[C@H]1O</smiles><smiles>[R10]C[C@H]1O[C@H](Oc2cc(O)ccc2C(=C)CCc2ccc(O)cc2)[C@H](O)[C@@H](O)[C@H]1O</smiles>

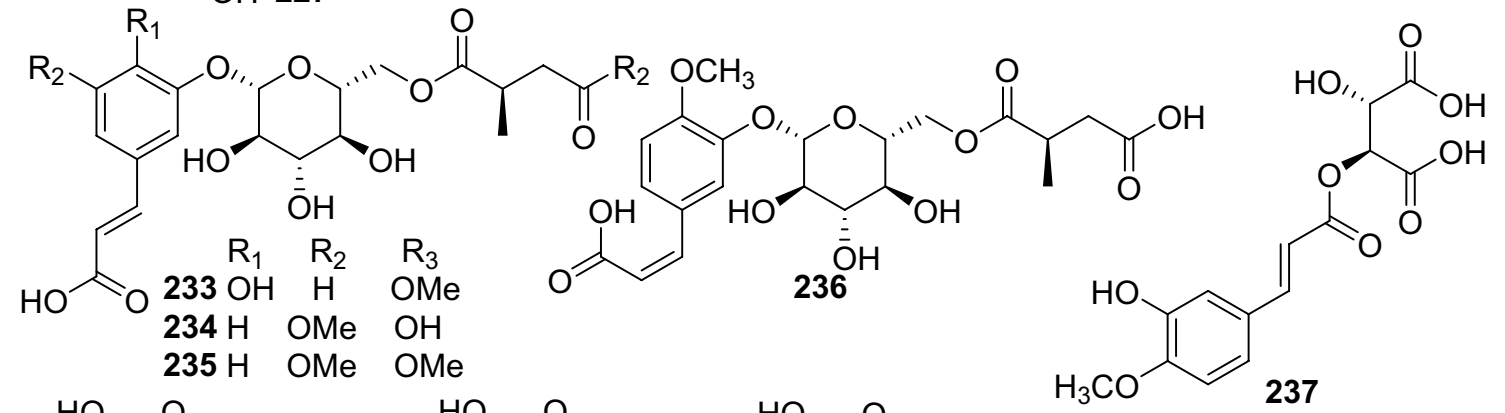<smiles>[R2]O[C@H]1C([R20])C[C@](O)(C(=O)O)C[C@@H]1O</smiles>

$238 \mathrm{R}_{1}=\mathrm{C}, \mathrm{R}_{2}=\mathrm{H}$

$239 \mathrm{R}_{1}=\mathrm{H}, \mathrm{R}_{2}=\mathrm{C}$

$240 R_{1}=H, R_{2}=A$

$\mathrm{MeO} Y \mathrm{O}$<smiles>[R20]O[C@H]1C[C@](O)(C(=C)C)C[C@H](O[R20])[C@@H]1O</smiles>

$245 R_{1}=A, R_{2}=H$

$246 \mathrm{R}_{1}=\mathrm{B}, \mathrm{R}_{2}=\mathrm{H}$

$247 R_{1}=C, R_{2}=H$

$248 \mathrm{R}_{1}=\mathrm{H}, \mathrm{R}_{2}=\mathrm{A}$

$249 \mathrm{R}_{1}=\mathrm{H}, \mathrm{R}_{2}=\mathrm{B}$<smiles>[R20]C1[C@H](O)CC(O)(C(=O)O)C[C@H]1O</smiles><smiles>[R]O[C@H]1[C@@H](O)C[C@@](O)(C(=O)O)C[C@H]1O</smiles>

$241 \mathrm{R}_{1}=\mathrm{C}, \mathrm{R}_{2}=\mathrm{H}$

$242 R_{1}=A, R_{2}=H$

$244 R=B$

$243 R_{1}=H, R_{2}=B$<smiles>O=C(O)/C=C/c1ccc(O[C@@H]2O[C@H](CO)[C@@H](O)[C@H](O)[C@H]2O)c(O)c1</smiles> 
Table 9 Information of phenylpropionic acids from Actinidia plants

\begin{tabular}{|c|c|c|c|c|}
\hline No. & Compound name & Species Refs. & Part & Bioactivity Refs. \\
\hline 220 & Caffeic acid & A. arguta $[135]$ & Fruits & $\begin{array}{l}\text { Anti-oxidation } \\
\text { (in vitro) [135] }\end{array}$ \\
\hline 221 & Caffeoyl- $\beta$-D-glucopyranoside & A. arguta $[135]$ & Fruits & $\begin{array}{l}\text { Anti-oxidation } \\
\text { (in vitro) [135] }\end{array}$ \\
\hline 222 & Trans-ferulic acid & A. polygama $[110]$ & Aerial parts & \\
\hline 223 & Coumaric acid 4-O-glucoside & A. polygama [110] & Aerial parts & \\
\hline 224 & Trans-phydroxycinnamic acid & A. chinensis [137] & Roots & \\
\hline 225 & Caffeic 3-O- $\beta$-D-glucopyranoside acid & A. deliciosa [89] & Pulps & \\
\hline 226 & Caffeic $4-\mathrm{O}-\beta$-D-glucopyranoside acid & A. deliciosa [89] & Pulps & \\
\hline 227 & 4,4'-Dihydroxyl-dihydrochalcone-2'-O- $\beta$-D-glucopyranoside & A. chinensis Plach [52] & Roots & \\
\hline 228 & Argutinoside A & A. arguta $[136]$ & Fruits & \\
\hline 229 & Argutinoside B & A. arguta $[136]$ & Fruits & \\
\hline 230 & Argutinoside C & A. arguta $[136]$ & Fruits & \\
\hline 231 & Argutinoside D & A. arguta $[136]$ & Fruits & \\
\hline 232 & Argutinoside E & A. arguta $[136]$ & Fruits & \\
\hline 233 & Argutinoside F & A. arguta $[136]$ & Fruits & \\
\hline 234 & Argutinoside $\mathrm{G}$ & A. arguta $[136]$ & Fruits & \\
\hline 235 & Argutinoside $\mathrm{H}$ & A. arguta $[136]$ & Fruits & \\
\hline 236 & Argutinoside I & A. arguta $[136]$ & Fruits & \\
\hline 237 & Fertaric acid & A. arguta $[138]$ & Fruits & \\
\hline 238 & Cryptochlorogenic acid & A. chinensis [137] & Roots & \\
\hline 239 & Neochlorogenic acid & A. chinensis [137] & Roots & $\begin{array}{l}\text { Anti-inflammatory } \\
\text { (in vitro) [139] } \\
\text { Antibacteria and antioxidation } \\
\text { (in vitro) [140] }\end{array}$ \\
\hline 240 & 3-O-Coumaroylquinic acid & A. chinensis [137] & Roots & \\
\hline 241 & Chlorogenic acid & A. deliciosa [89] & Pulp & $\begin{array}{l}\text { Antitumor (in vitro) [141] } \\
\text { Anti-inflammatory } \\
\text { (in vitro) [142] }\end{array}$ \\
\hline 242 & 5- Trans-p-coumaroylmalic acid & A. chinensis Radix [50] & Roots & \\
\hline 243 & 5- Cis-p-coumaroylquinic acid & A. chinensis Radix [50] & Roots & \\
\hline 244 & 4-O-Cis-p-coumaroylquinic acid & A. chinensis Radix [50] & Roots & \\
\hline 245 & 3-O-Trans-p-coumaroyl quinic acid methyl ester & A. arguta $[136]$ & Fruits & $\begin{array}{l}\text { Inhibiting NF- } \kappa \text { B transcription } \\
\quad \text { (in vitro) }[136]\end{array}$ \\
\hline 246 & 3-O-Cis-p-coumaroyl quinic acid methyl ester & A. arguta $[136]$ & Fruits & \\
\hline 247 & 3-O-Trans-p-caffeoyl quinic acid methyl ester & A. arguta [136] & Fruits & \\
\hline 248 & 5-O-Trans-p-coumaroyl quinic acid methyl ester & A. arguta $[136]$ & Fruits & \\
\hline 249 & 5-O-Cis-p-coumaroyl quinic acid methyl ester & A. arguta [136] & Fruits & \\
\hline 250 & 5-O-Trans-p-caffeoyl quinic acid methyl ester & A. arguta $[136]$ & Fruits & \\
\hline 251 & 5-O-Cis-p-caffeoyl quinic acid methyl ester & A. arguta [136] & Fruits & \\
\hline 252 & 3-O-Trans-p-caffeoyl quinic acid butyl ester & A. arguta $[136]$ & Fruits & \\
\hline 253 & 4-O-Trans-p-caffeoyl quinic acid butyl ester & A. arguta [136] & Fruits & \\
\hline 254 & 5-O-Trans-p-coumaroyl quinic acid butyl ester & A. arguta [136] & Fruits & \\
\hline 255 & 5-O-Trans-p-caffeoyl quinic acid butyl ester & A. arguta $[136]$ & Fruits & \\
\hline 256 & 3-O-Trans-p-coumaroyl shikimic acid & A. arguta $[136]$ & Fruits & \\
\hline 257 & 3-O-Cis-p-coumaroyl shikimic acid & A. arguta $[136]$ & Fruits & \\
\hline
\end{tabular}

anti-inflammatory component from the EtOAc fraction of $A$. polygama Max. It potently inhibited nitric oxide production (30.6\% inhibition at $1 \mu \mathrm{g} / \mathrm{mL}$ ) in lipopolysaccharide (LPS)stimulated RAW264.7 cells and $\beta$-hexosaminidase release
(91.8\% inhibition at $1 \mu \mathrm{g} / \mathrm{mL}$ ) in IgE-sentized RBL-2H3 cells [182].

In summary, this review focused on the biological components and related pharmacological activities of various 
<smiles>COc1cc2ccc(=O)oc2cc1O</smiles><smiles>COc1cc2ccc(=O)oc2c(O)c1O[C@H]1O[C@H](CO)[C@@H](O)[C@H](O)[C@H]1O</smiles>

262<smiles>COc1c(OC2O[C@H](CO)[C@@H](O)[C@H](O)[C@H]2O)c(O)c2oc(=O)ccc2c1O</smiles>

265<smiles>O=c1ccc2cc(O[C@@H]3O[C@H](CO)[C@@H](O)[C@H](O)[C@H]3O)c(O)cc2o1</smiles>

263<smiles>COc1cc2oc(=O)ccc2c(OC2OC(COC(C)=O)[C@@H](O)[C@H](O)[C@H]2O)c1OC</smiles>

266<smiles>COc1cc2ccc(=O)oc2c(O[C@@H]2O[C@H](CO)[C@@H](O)[C@H](O)[C@H]2O)c1O</smiles>

264

$267 \mathrm{R}=\mathrm{OH}, \mathrm{R}_{1}=\mathrm{H}$

$268 \mathrm{R}=\mathrm{R}_{1}=\mathrm{OMe}$

Fig. 11 Structures of coumarins 258-268 from Actinidia plants

Table 10 Information of coumarins from Actinidia plants

\begin{tabular}{|c|c|c|c|c|}
\hline No. & Compound name & Species Refs. & Part & Bioactivity Refs. \\
\hline 258 & Umbelliferone & A. polygama [109] & Leaves & $\begin{array}{l}\text { Anti-tumor (in vitro) [143] } \\
\text { Anti-inflammatory (in vitro) [144] } \\
\text { Antioxidant (in vitro) [149] } \\
\text { Antidiabetic (in vitro and in vivo) [146] } \\
\text { Immunomodulatory(in vitro) [148] }\end{array}$ \\
\hline 259 & Esculetin & A. arguta [135] & Fruits & $\begin{array}{l}\text { Anti-tumor (in vitro) [154] } \\
\text { Anti-oxidant and anti-inflammatory (in vivo) [155] }\end{array}$ \\
\hline 260 & Fraxetin & A. deliciosa $[150]$ & Roots & $\begin{array}{l}\text { Anti-inflammatory (in vitro) [151] } \\
\text { Anti-tumor (in vitro) [156] }\end{array}$ \\
\hline 261 & Isoscopoletin & A. deliciosa $[150]$ & Roots & Anti-inflammatory [151] \\
\hline 262 & Isofraxoside & A. chinensis [137] & Roots & \\
\hline 263 & Esculin & A. deliciosa $[152]$ & Stems & \\
\hline 264 & Fraxin & A. chinensis [152] & Fruits & $\begin{array}{l}\text { Anti-inflammatory (in vivo) [157] } \\
\text { Antitumor (in vitro) [153] }\end{array}$ \\
\hline 265 & $\begin{array}{l}\text { 5-Hydroxy-6-methoxy-7-O- } \beta \text {-D- } \\
\text { glycopyranosylcoumarin }\end{array}$ & A. chinensis Radix [50] & Roots & \\
\hline 266 & $\begin{array}{l}\text { 6'-Acetoxy-8- } \beta \text {-D-glucopyranosyloxy-7- } \\
\text { hydroxy-6-methoxy-coumarin }\end{array}$ & A. chinensis Radix [50] & Roots & \\
\hline 267 & 6-Hydroxy7-( $\beta$-D-glucopyranosyloxy) coumarin & A. deliciosa [89] & Peels & \\
\hline 268 & $\begin{array}{l}\text { 6,8-Dimethoxy-7-( } \beta \text {-D-glucopyranosyloxy) cou- } \\
\text { marin }\end{array}$ & A. deliciosa [89] & Peels & \\
\hline
\end{tabular}


<smiles>COc1cc(C2Oc3ccc(CCCO)cc3[C@H]2CO)ccc1O[C@@H]1O[C@H](CO)[C@@H](O)[C@H](O)[C@H]1O</smiles><smiles>COc1cc(C2Oc3ccc(CCCO)cc3C2CO)ccc1OC1OC[C@@H](O)[C@H](O)[C@H]1O</smiles>

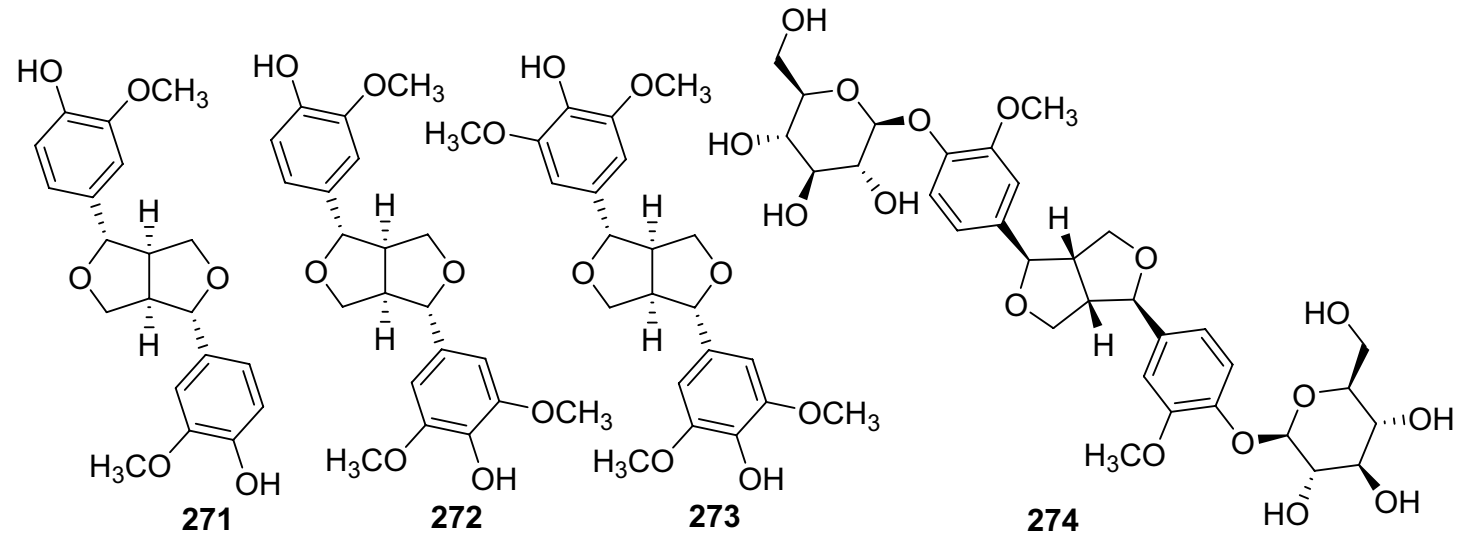

Fig. 12 Structures of lignans 269-274 from Actinidia plants

Table 11 Information of of lignans from Actinidia plants

\begin{tabular}{|c|c|c|c|c|c|}
\hline No. & Compound name & Species Refs. & Part & & $\begin{array}{l}\text { Cytotoxicity } \\
\text { Refs. }\end{array}$ \\
\hline 269 & Urolingoside & A. polygama [110] & Aerial parts & & \\
\hline 270 & 4'-O- $\beta$-Dxylopyranoside & A. chinensis Radix [50] & & Roots & \\
\hline 271 & (+)-Pinoresinol & A. arguta [53] & Roots & & $\begin{array}{l}\text { Antifungal (in } \\
\text { vitro) [158] } \\
\text { Anti-inflamma- } \\
\text { tory (in vitro) } \\
\text { [159] } \\
\text { Antioxidation } \\
\text { (in vitro) [160] } \\
\text { Hypoglycemic } \\
\text { (in vitro) [161] } \\
\text { Anti-tumor (in } \\
\text { vitro) [162] }\end{array}$ \\
\hline 272 & (+)-Medioresinol & A. arguta [53] & Roots & & \\
\hline 273 & $(-)$-Syringaresinol & A. arguta [53] & Roots & & \\
\hline 274 & Pinoresinol diglucoside & A. arguta $[138]$ & Leaves & & $\begin{array}{l}\text { Inhibiting } \\
\quad \alpha \text {-glucosidase } \\
\text { (in vitro) [138] }\end{array}$ \\
\hline
\end{tabular}


<smiles>OCCc1ccc(O)cc1</smiles>

275<smiles>CC1(C)CCc2cc(C(=O)O)ccc2O1</smiles>

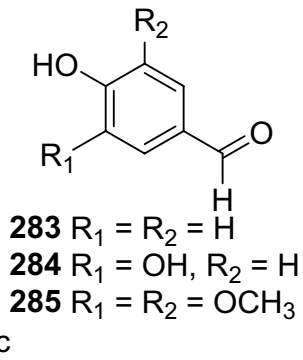<smiles>Cc1cc(O)c(C(=O)c2c(O)cccc2O)c(C(=O)O)c1</smiles><smiles>COC(=O)Cc1c(O)cc(O)cc1OC(=O)c1ccccc1</smiles><smiles>[R]c1cc(C(=O)O)ccc1O</smiles>

$279 \mathrm{R}=\mathrm{OH}$

$280 \mathrm{R}=\mathrm{H}$

$281 \mathrm{R}=\mathrm{OCH}_{3}$

$282 \mathrm{R}=0-1-\beta$-D-glc<smiles>COc1cc(C(=O)O)ccc1O[C@@H]1O[C@H](CO)[C@@H](O)[C@H](O)[C@H]1O</smiles>

$\mathrm{OH}$<smiles>COc1cc(O[C@@H]2O[C@H](CO)[C@@H](O)[C@H](O)[C@H]2O)ccc1C(=O)O</smiles><smiles>COc1cc(O)ccc1O[C@@H]1O[C@H](CO)[C@@H](O)[C@H](O)[C@H]1O</smiles>

288<smiles>COc1cc([C@@H](O)[C@H](O)c2ccc(O)c(OC)c2)ccc1O</smiles><smiles>COc1cc([C@H](O)[C@H](O)c2ccc(O)c(OC)c2)ccc1O</smiles><smiles>[R20]Oc1cc([R17])c2c(c1)O[C@@H]1[C@H](C2)O[C@]2(C)OC(=O)C[C@@H]12</smiles>

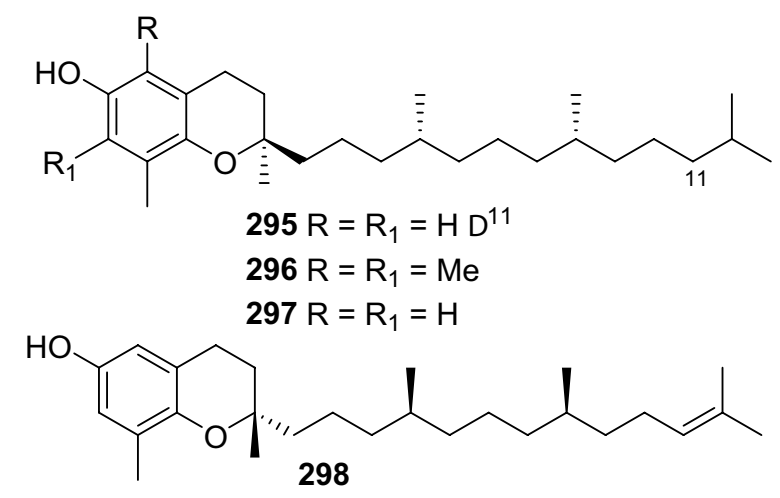

290

$291 \mathrm{R}_{1}=\mathrm{H}, \mathrm{R}_{2}=\mathrm{H}$

$292 \mathrm{R}_{1}=\mathrm{H}, \mathrm{R}_{2}=\mathrm{Me}$

$293 \mathrm{R}_{1}=\mathrm{Me}, \mathrm{R}_{2}=\mathrm{Me}$

$294 R_{1}=H, R_{2}=A c$

Fig. 13 Structures of simple phenols 275-298 from Actinidia plants

parts of Actinidia plants, including triterpenoids, steroids, flavonoids, catechins, coumarins, lignans, phenols, and other small organic molecules. A total of 325 molecules have been collected in this review. Most of the active molecules are derived from the roots of Actinidia plants, while triterpenes and flavonoids are the most important types regardless of the number of compounds and their biological activity significance. The stems, leaves, fruit galls, and other parts of kiwi are mainly rich in flavonoids, phenylpropionic acids, and other small molecule compounds. Currently, these chemical components are not structurally novel. In addition, there are few in-depth researches on pharmacological activities of the bioactive compounds. Therefore, research on the chemical constituents of Actinidia plants is still promising. We hope that this review can provide positive information for the further exploration of the chemical components and their biological activities of Actinidia plants. 
Table 12 Information of simple phenols from Actinidia plants

\begin{tabular}{|c|c|c|c|c|}
\hline No. & Compound name & Species Refs. & Part & Bioactivity Refs. \\
\hline 275 & Tyrosol & A. arguta $[168]$ & Roots & Anti-inflammatory (in vitro) [151] \\
\hline 276 & 2,2-Dimethyl-6-chromancarboxylic acid & A. deliciosa $[150]$ & Roots & \\
\hline 277 & Monodictyphenone & A. chinensis Radix [50] & Roots & $\begin{array}{l}\text { Inhibiting protein tyrosine phosphatase (in vitro) } \\
\text { [169] }\end{array}$ \\
\hline 278 & $\begin{array}{l}\text { 3,5-Dihydroxy-2-(methoxycarbonyl methyl) } \\
\text { phenyl 3,4-dihydroxybenzoate }\end{array}$ & A. chinensis [167] & Leaves & \\
\hline 279 & Protocatechuic acid & A. arguta $[135]$ & Fruits & $\begin{array}{l}\text { Anti-inflammatory and antioxidant (in vitro and } \\
\text { in vivo) [163] } \\
\text { Neuroprotective (in vitro) }[164] \\
\text { Anti-proliferative (in vitro) [165] }\end{array}$ \\
\hline 280 & 4-Hydroxy benzoic acid & A. arguta $[92]$ & Roots & \\
\hline 281 & Vanillic acid & A. deliciosa $[150]$ & Roots & \\
\hline 282 & $\begin{array}{l}\text { 4-( } \beta \text {-D-Glucopyranosyloxy)-3-hydroxybenzoic } \\
\text { acid }\end{array}$ & A. chinensis Radix [50] & Roots & \\
\hline 283 & p-Hydroxybenzaldehyde & A. chinensis Radix [50] & Roots & \\
\hline 284 & Tachioside & A. deliciosa $[150]$ & Roots & \\
\hline 285 & Syringaldehyde & A. chinensis Planch [105] & Roots & Anti-inflammatory (in vitro) [170] \\
\hline 286 & Vanillic acid 4-O- $\beta$-D-glucopyranoside & A. deliciosa $[150]$ & Roots & \\
\hline 287 & Protocatechualdehyde & A. chinensis [171] & Roots & Antioxidant and anti-inflammatory (in vitro) [172] \\
\hline 288 & $\begin{array}{l}\text { 4-Hydroxy-2-methoxyphenyl- } \beta \text {-D- } \\
\text { glucopyranoside }\end{array}$ & $\begin{array}{l}\text { A. macrosperma } \\
\text { [66] }\end{array}$ & Roots & \\
\hline 289 & $\begin{array}{l}\text { Erythro-1,2-bis-(4-hydroxy-3-methoxyphenyl)- } \\
\text { 1,3-propanediol }\end{array}$ & A. chinensis [171] & Roots & \\
\hline 290 & $\begin{array}{l}\text { Threo-1,2-bis-(4-hydroxy-3-methoxyphenyl)- } \\
\text { 1,3-propanediol }\end{array}$ & A. chinensis [171] & Roots & \\
\hline 291 & Planchols A & A. chinensis Planch [94] & Roots & Against P-388 and A-549 cell lines (in vitro) [94] \\
\hline 292 & Planchols B & A. chinensis Planch [94] & Roots & Against P-388 and A-549 cell lines (in vitro) [94] \\
\hline 293 & Planchols C & A. chinensis Planch [94] & Roots & Against P-388 and A-549 cell lines (in vitro) [94] \\
\hline 294 & Planchols D & A. chinensis Planch [94] & Roots & Against P-388 and A-549 cell lines (in vitro) [94] \\
\hline 295 & $\begin{array}{l}\text { 2,8-Dimethyl-2-(4,8,12-trimethyltridec-11-enyl) } \\
\text { chroman-6-ol }\end{array}$ & A. deliciosa [89] & Peels & \\
\hline 296 & $\alpha$-Tocopherol & A. deliciosa [89] & Peels & \\
\hline 297 & $\delta$-Tocopherol & A. deliciosa [89] & Peels & \\
\hline 298 & $\begin{array}{l}\text { 2,8-Dimethyl-2-(4,8,12-trimethyltridec-11-enyl) } \\
\text { chroman-6-ol }\end{array}$ & A. chinensis [173] & Peels & \\
\hline
\end{tabular}




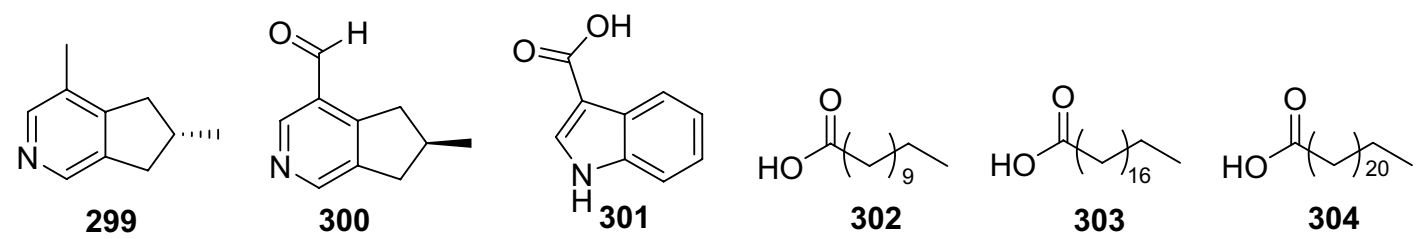<smiles>CC/C=C\C/C=C/C/C=C\CC(C)=O</smiles><smiles>CCCCC[C@H](O)/C=C/C=C/CC(C)=O</smiles><smiles>CCCCCC[C@H](O)C/C=C\CC(=O)O</smiles><smiles>CCCCC(C)(CCC)C(=O)O</smiles><smiles>CCCCCC(=O)OC1OC(CO)[C@H](O)[C@H](O)[C@H]1O</smiles><smiles>OCC1OCCCC1O</smiles><smiles>CCCCCCCCC=CCC(O)CCC</smiles>

309<smiles>OCC(O)CO</smiles><smiles>C/C=C\CC/C=C\CCCCCCC</smiles><smiles>CCCCO[C@]1(CO)OC[C@@H](O)[C@H](O)[C@H]1O</smiles><smiles>CCO[C@]1(O)OC(O)[C@@H](O)[C@@H]1O[C@H]1O[C@H](CO)[C@@H](O)[C@H](O)[C@H]1O</smiles><smiles>O=C(O)CCC(=O)O</smiles><smiles>OC[C@H]1O[C@H](O[C@@H]2[C@@H](CO)OC(O)[C@H](O)[C@H]2O)[C@H](O)[C@@H](O)[C@@H]1O</smiles>

324

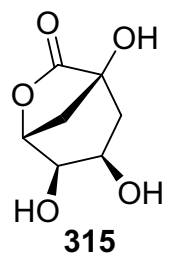<smiles>[R]OC(=O)CC(O)(CC([R20])=O)C([R6])=O</smiles>
$316 \mathrm{n}-\mathrm{Bu} \mathrm{H} \quad \mathrm{H}$ $317 \mathrm{n}-\mathrm{Bu} \mathrm{H} \quad \mathrm{Me}$ $318 \mathrm{Me} \mathrm{H} \quad \mathrm{Me}$ $319 \mathrm{Me} \mathrm{Me} \mathrm{Me}$ $320 \mathrm{H}$ Et $\mathrm{Me}$ $321 \mathrm{Me}$ Et Me

Fig. 14 Structures of other molecules 299-325 from Actinidia plants 
Table 13 Information other molecules from Actinidia plants

\begin{tabular}{|c|c|c|c|c|}
\hline No. & Compound name & Species Refs. & Part & Bioactivity Refs. \\
\hline 299 & Actinidine & A. polygama $[174]$ & Leaves and galls & Anti-obesity (in vivo) [183] \\
\hline 300 & Boschniakine & A. arguta [175] & & \\
\hline 301 & Indole-3-carboxylic acid & A. chinensis Planch [184] & Roots & \\
\hline 302 & Undecanoic acid & A. deliciosa [185] & Roots & $\begin{array}{l}\text { Anti-inflammatory } \\
\text { (in vivo) [186] }\end{array}$ \\
\hline 303 & $\mathrm{n}$-Stearic acid & A. deliciosa [150] & Roots & \\
\hline 304 & Tetracosanoic acid & A. eriantha Benth [41] & Roots & \\
\hline 305 & $\alpha$-Linolenic acid & A. polygama $[176]$ & Fruits & $\begin{array}{l}\text { Anti-inflammatory } \\
\text { (in vivo) [177] } \\
\text { Anti-tumor (in vivo) [178] } \\
\text { Anti-hyperlipidemic (in vivo) [179] } \\
\text { Anti-diabetic (in vivo) [180] } \\
\text { Anti-fungal (in vitro) [181] }\end{array}$ \\
\hline 306 & $\begin{array}{l}(9 Z, 11 E)-13 \text {-Hydroxy-9,11-octadeca- } \\
\text { dienoic acid }\end{array}$ & A. chinensis Radix [50] & Roots & \\
\hline 307 & Ricinoleic acid & A. chinensis Radix [50] & Roots & \\
\hline 308 & Lignoceric acid & A. chinensis Planch [187] & Roots & \\
\hline 309 & Stearyl- $\beta$-D-glucopyranoside & A. chinensis Planch [187] & Roots & \\
\hline 310 & Dotriacontanic acid & A. chinensis Planch [52] & Roots & \\
\hline 311 & Sphingolipid & A. chinensis Planch [52] & Roots & \\
\hline 312 & Actinidiamide & A. polygama [182] & Fruits & $\begin{array}{l}\text { Anti-inflammatory } \\
\text { (in vitro) [182] }\end{array}$ \\
\hline 313 & $n$-Butyl-O- $\beta$-D-fructopyranoside & A. deliciosa $[188]$ & Roots & \\
\hline 314 & Sucrose & A. chinensis Planch [187] & Roots & \\
\hline 315 & $\gamma$-Quinide & A. chinensis Planch [187] & Roots & \\
\hline 316 & 1-Methyl-5-ethyl citrate & A. arguta [136] & Fruits & \\
\hline 317 & 1,6-Dimethyl citrate & A. arguta [136] & Fruits & \\
\hline 318 & 1,5,6-Trimethyl citrate & A. arguta $[136]$ & Fruits & \\
\hline 319 & 1,6-Dimethyl-5-ethyl citrate & A. arguta $[136]$ & Fruits & \\
\hline 320 & 6-Butyl citrate & A. arguta $[136]$ & Fruits & \\
\hline 321 & 1-Methyl-6-butyl citrate & A. arguta [136] & Fruits & \\
\hline 322 & Succinic acid & A. kolomikta [189] & Leaves & \\
\hline 323 & Meso-inositol & A. kolomikta [189] & Leaves & \\
\hline 324 & Maltose & A. kolomikta [189] & Leaves & \\
\hline 325 & $\alpha$-Kolomiktriose & A. kolomikta $[190]$ & Roots & \\
\hline
\end{tabular}

Funding This work was financially supported by the National Natural Science Foundation of China (22177139) and the Scientific Research Program of Hubei Provincial Department of Education, China (D20183001).

Open Access This article is licensed under a Creative Commons Attribution 4.0 International License, which permits use, sharing, adaptation, distribution and reproduction in any medium or format, as long as you give appropriate credit to the original author(s) and the source, provide a link to the Creative Commons licence, and indicate if changes were made. The images or other third party material in this article are included in the article's Creative Commons licence, unless indicated otherwise in a credit line to the material. If material is not included in the article's Creative Commons licence and your intended use is not permitted by statutory regulation or exceeds the permitted use, you will need to obtain permission directly from the copyright holder. To view a copy of this licence, visit http://creativecommons.org/licenses/by/4.0/.

\section{References}

1. S.J. Henare, S.M. Rutherfurd, L.N. Drummond, V. Borges, M.J. Boland, P.J. Moughan, Food Chem. 130, 67-72 (2012)

2. G. Du, M. Li, F. Ma, D. Liang, Food Chem. 113, 557-562 (2009)

3. C.V. Garcia, S.Y. Quek, R.J. Stevenson, R.A. Winz, J. Agric. Food Chem. 59, 8358-8365 (2011)

4. X. Xiao, R. Sun, S. Jiang, T. Du, G. Yang, F. Ye, China Med. Hered. 11, 157-159 (2014)

5. Y.N. Zhang, L. Liu, C.Q. Ling, Chin J. Chin. Mater. Med. 31, 918-920 (2006)

6. T. Fang, M. He, J. Xia, J. Hou, L. Wang, M. Zheng, X. Wang, J. Xia, Cell Biol. Toxicol. 32, 499-511 (2016)

7. C. Huang, Z. Zhang, G. Li, J. Zhou, Plant Divers. Resour. 10, 93-100 (1988)

8. A. Manayi, M. Nikan, N. Nobakht-Haghighi, M. Abdollahi, Curr. Med. Chem. 25, 4866-4875 (2018) 
9. A.K. Singh, H. Pandey, P.W. Ramteke, S.B. Mishra, Lat. Am. J. Pharm. 38, 513-517 (2019)

10. A.B. Ramos-Hryb, N. Platt, A.E. Freitas, I.A. Heinrich, M.G. Lopez, R.B. Leal, M.P. Kaster, A.L.S. Rodrigues, Neurochem. Res. 44, 2843-2855 (2019)

11. P.D. Moon, N.R. Han, J.S. Lee, H.M. Kim, H.J. Jeong, Int. J. Mol. Med. 43, 2252-2258 (2019)

12. X. Jiang, T. Li, R.H. Liu, J. Agric. Food Chem. 64, 1806-1816 (2016)

13. D.S. Jang, G.Y. Lee, J. Kim, Y.M. Lee, J.M. Kim, Y.S. Kim, J.S. Kim, Arch. Pharmacal. Res. 31, 666-670 (2008)

14. M. Takaya, M. Nomura, T. Takahashi, Y. Kondo, K.T. Lee, S. Kobayashi, Anticancer Res. 29, 995-1000 (2009)

15. J.H. Won, K.S. Chung, E.Y. Park, J.H. Lee, J.H. Choi, L.A. Tapondjou, H.J. Park, M. Nomura, A.H.E. Hassan, K.T. Lee, Molecules 23, 3306 (2018)

16. K.W. Woo, S.U. Choi, K.H. Kim, K.R. Lee, J. Braz. Chem. Soc. 26, 1450-1456 (2015)

17. K.M. Shin, R.K. Kim, T.L. Azefack, L. David, S.B. Luc, M.I. Choudhary, H.J. Park, J.W. Choi, K.T. Lee, Planta Med. 70, 803-807 (2004)

18. Y. Sashida, K. Ogawa, N. Mori, T. Yamanouchi, Phytochemistry 31, 2801-2804 (1992)

19. Y.X. Xu, Z.B. Xiang, Y.S. Jin, Y. Shen, H.S. Chen, Fitoterapia 81, 920-924 (2010)

20. Y.X. Xu, Z.B. Xiang, X.J. Chen, H.S. Chen, Acad. J. Second Mil. Med. Univ. 32, 749-753 (2011)

21. H.L. Xin, Y.C. Wu, Y.F. Xu, Y.H. Su, Y.N. Zhang, C.Q. Ling, Chin. J. Nat. Med. 8, 260-263 (2010)

22. H.L. Xin, X.Q. Yue, Y.F. Xu, Y.C. Wu, Y.N. Zhang, Y.Z. Wang, C.Q. Ling, Helv. Chim. Acta. 91, 575-580 (2008)

23. Y. Sashida, K. Ogawa, T. Yamanouchi, H. Tanaka, Y. Shoyama, I. Nishioka, Phytochemistry 35, 377-380 (1994)

24. D. Li, W. Li, K. Higai, K. Koike, J. Nat. Med. 68, 427-431 (2014)

25. X.Y. Zhang, Y. Zhou, Z.P. Wei, J. Shen, L.K. Wang, Z.Q. Ma, X. Zhang, Pest Manag. Sci. 74, 1630-1636 (2018)

26. H. Takazawa, K. Yoshimura, A. Ikuta, K. Kawaguchi, Plant Biotechnol. 19, 181-186 (2002)

27. S.Y. Du, H.F. Huang, X.Q. Li, L.X. Zhai, Q.C. Zhu, K. Zheng, X. Song, C.S. Xu, C.Y. Li, Y. Li, Z.D. He, H.T. Xiao, Chin. Med. 15, $43(2020)$

28. G.C. Bonel-Perez, A. Perez-Jimenez, I. Gris-Cardenas, A.M. Parra-Perez, J.A. Lupianez, F.J. Reyes-Zurita, E. Siles, R. Csuk, J. Peragon, E.E. Rufino-Palomares, Molecules 25, 4254 (2020)

29. J. Carmo, P. Cavalcante-Araujo, J. Silva, J. Ferro, A.C. Correia, V. Lagente, E. Barreto, Molecules 25, 4982 (2020)

30. X.F. Zhou, P. Zhang, H.F. Pi, Y.H. Zhang, H.L. Ruan, H. Wang, J.Z. Wu, Chem. Biodivers. 6, 1202-1207 (2009)

31. E.H. Lahlou, N. Hirai, T. Kamo, M. Tsuda, H. Ohigashi, Biosci. Biotechnol. Biochem. 65, 480-483 (2001)

32. L.B. Wei, S.Y. Ma, H.X. Liu, C.S. Huang, N. Liao, Chem. Biodivers. 15, e1700454 (2018)

33. N.A. Dangroo, J. Singh, A.A. Dar, N. Gupta, P.K. Chinthakindi, A. Kaul, M.A. Khuroo, P.L. Sangwan, Eur. J. Med. Chem. 120, 160-169 (2016)

34. J.H. Ahn, Y. Park, S.W. Yeon, Y.H. Jo, Y.K. Han, A. Turk, S.H. Ryu, B.Y. Hwang, K.Y. Lee, M.K. Lee, J. Nat. Prod. 83, 14161423 (2020)

35. C.S. Huang, S.Y. Ma, H.X. Liu, Q. Lu, C.S. Huang, H.X. Liu, L.B. Wei, L.G. Shi, N. Liao, L.B. Wei, China J. Chin. Mater. Med. 42, 2714-2718 (2017)

36. L.P. Qu, G.Y. Zheng, Y.H. Su, H.Q. Zhang, Y.L. Yang, H.L. Xin, C.Q. Ling, Int. J. Mol. Sci. 13, 14865-14870 (2012)

37. Y. Shi, H. Wang, B. Ma, Chin. Tradit. Herb. Drugs. 24, 386-387 (1993)
38. S.C. Dong, T.Y. Shin, J.S. Eun, D.K. Kim, H. Jeon, Arch. Pharmacal. Res. 34, 425-436 (2011)

39. D. Xu, A. Qiu, N. Tang, G. Liu, Y. Lai, CN101502542.

40. C. Huang, G. Li, H. Fan, Z. Zhang, J. Zhou, Plant Divers. Resour. 8, 489-491 (1986)

41. C. Huang, X. Chen, Nat. Prod. Res. Dev. 4, 27-30 (1992)

42. L. Meng, C. Huang, H. Liu, X. Chen, Chin. Tradit. Herb. Drugs 32, 1544-1546 (2009)

43. L. Wei, C. Huang, H. Liu, X. Chen, Technol. Dev Chem. Ind. 38, 1-3 (2009)

44. Y. Cui, X.M. Zhang, J.J. Chen, Y. Zhang, X.K. Lin, L. Zhou, China J. Chin. Mater. Med. 32, 1663-1665 (2007)

45. W.J. Zhu, D.H. Yu, M. Zhao, M.G. Lin, Q. Lu, Q.W. Wang, Y.Y. Guan, G.X. Li, X. Luan, Y.F. Yang, X.M. Qin, C. Fang, G.H. Yang, H.Z. Chen, Anti-Cancer Agents Med. Chem. 13, 195-198 (2013)

46. Y. Qin, C. Huang, X. Chen, M. Cai, H. Liu, Chin. Tradit. Herb. Drugs. 30, 323-326 (1999)

47. F. Lu, L. Zhao, L. Zheng, L. Lu, Cent. S. Pharm. 12, 165-168 (2014)

48. Y.D. Xu, L. Yin, Chin. Tradit. Herb. Drugs. 44, 935-937 (2013)

49. S. Bei, C. Huang, X. Chen, Nat. Prod. Res. Dev. 9, 15-18 (1997)

50. C. Nie, J. Yang, D. Wu, L. Wan, G. Liang, Chem. Res. Chin. Univ. 35, 823-829 (2019)

51. C. Ye, M. Jin, Y. Zhou, W. Zhou, G. Li, Chem. Nat. Compd. 55, 975-977 (2019)

52. Y.X. Xu, Z.B. Xiang, Y.S. Jin, W. Xu, L.N. Sun, W.S. Chen, HSJC Chem. Biodivers. 13, 1454-1459 (2016)

53. J.I. Whang, H.I. Moon, O.P. Zee, Saengyak Hakhoechi. 31, 357-365 (2000)

54. Y. Han, Z. Tong, C. Wang, X. Li, G. Liang, Eur. J. Pharmacol. 893, 173811 (2021)

55. S. Zhang, Y. Liu, X. Wang, Z. Tian, D. Qi, Y. Li, H. Jiang, Int. J. Mol. Med. 46, 2019-2034 (2020)

56. P.M. Edathara, S. Chintalapally, V.K.K. Makani, C. Pant, S. Yerramsetty, M.D. Rao, M.P. Bhadra, Gene 771, 145370 (2021)

57. W. Zhou, X. Zeng, X. Wu, Med. Sci. Monit. 26, e921606 (2020)

58. J.M. Castellano, S. Garcia-Rodriguez, J.M. Espinosa, M.C. Millan-Linares, M. Rada, J.S. Perona, Biomolecules 9, 683 (2019)

59. W.J.A. Musa, B. Situmeang, J. Sianturi, Int. J. Food Prop. 22, 1439-1444 (2019)

60. L.Y. Mooi, N. Abdul Wahab, N.H. Lajis, A.M. Ali, Chem. Biodivers. 7, 1267-1275 (2010)

61. L. Huang, T. Guan, Y. Qian, M. Huang, X. Tang, Y. Li, H. Sun, Eur. J. Pharmacol. 672, 169-174 (2011)

62. J.G. Wu, L. Ma, S.H. Lin, Y.B. Wu, J. Yi, B.J. Yang, J.Z. Wu, K.H. Wong, J. Ethnopharmacol. 203, 1-10 (2017)

63. F. A. Ma, D. L. Wu, F. Q. Xu, W. Zhang, Y. S. R, Chin. Tradit. Pat. Med. 38, 591-593 (2016).

64. H. Zhao, B.Z. Wang, B.R. Ma, J.Y. Sun, Chin. Pharm. J. 29, 523-524 (1994)

65. Y. Lai, D. Xu, J. Chin. Med. Mater. 30, 166-168 (2007)

66. L. Ding, S. Wang, Z. Wang, China J. Chin. Mater. Med. 32, 1893-1895 (2007)

67. J. Lu, R. Yang, M. Gui, Y. Jin, J. Dong, X. Li, Chin. Pharm. J. 44, 1215-1217 (2009)

68. L.J. Zhu, S.T. Xiang, X.H. Wang, J. Zhao, Z.I. Tan, J.E. Yi, J. Tradit. Chin. Vet. Med. 35, 18-22 (2016)

69. C.G. Farcas, C. Dehelean, I.A. Pinzaru, M. Mioc, V. Socoliuc, E.A. Moaca, S. Avram, R. Ghiulai, D. Coricovac, I. Pavel, P.K. Alla, O.M. Cretu, C. Soica, F. Loghin, Int. J. Nanomed. 15, 8175-8200 (2020)

70. L. Kun, J.Y. Wang, L. Zhang, Y.Y. Pan, X.Y. Chen, Y. Yuan, Int. J. Immunopathol. Pharmacol. 34, 2058738420945078 (2020)

71. H. Wang, F. Dong, Y. Wang, X.A. Wang, D. Hong, Y. Liu, J. Zhou, Acta Biochim. Biophys. Sin. 52, 200-206 (2020) 
72. Q. Wang, Y. Li, L. Zheng, X. Huang, Y. Wang, C.H. Chen, Y.Y. Cheng, S.L. Morris-Natschke, K.H. Lee, A.C.S. Med, Chem. Lett. 11, 2290-2293 (2020)

73. G.A. Birgani, A. Ahangarpour, L. Khorsandi, H.F. Moghaddam, Braz. J. Pharm. Sci. 54, e17171 (2018)

74. Z. Zhou, C. Zhu, Z. Cai, F. Zhao, L. He, X. Lou, X. Qi, Oncol. Lett. 15, 7319-7327 (2018)

75. Y.H. Han, J.G. Mun, H.D. Jeon, J.Y. Kee, S.H. Hong, Nutrients 12, 66 (2019)

76. F. Yin, F. Feng, L. Wang, Z. Li, X. Wang, Y. Cao, Cell Death Dis. 10, 672 (2019)

77. F. Murai, M. Tagawa, Planta Med. 37, 234-240 (1979)

78. T. Sakai, K. Nakajima, T. Sakan, Bull. Chem. Soc. Jpn. 53, 3683-3686 (1980)

79. S. Isoe, T. Ono, S.B. Hyeon, T. Sakan, Tetrahedron Lett. 9, 5319$5323(1968)$

80. F. Murai, M. Tagawa, H. Ohishi, Planta Med. 58, 112-113 (1992)

81. S. Jain, A. Ganeshpurkar, N. Dubey, Pharmacogn. Commun. 10, 134-135 (2020)

82. K. Koc, F. Geyikoglu, O. Cakmak, A. Koca, Z. Kutlu, F. Aysin, A. Yilmaz, H. Askin, Naunyn-Schmiedeberg's Arch. Pharmacol. 394, 469-479 (2021)

83. J.Y. Ye, L. Li, Q.M. Hao, Y. Qin, C.S. Ma, Korean J. Physiol. Pharmacol. 24, 39-46 (2020)

84. F. Zhang, Z. Liu, X. He, Z. Li, B. Shi, F. Cai, Drug Deliv. 27, 1329-1341 (2020)

85. L. Karthik, B. Vijayakumar, Int. J. Pharm. Phytopharm. Res. 10, $8-21(2020)$

86. A. Sen, P. Dhavan, K.K. Shukla, S. Singh, G. Tejovathi, Sci. Secure J. Biotechnol. 1, 9-13 (2012)

87. S. Babu, S.J.B. Jayaraman, Pharmacotherapy 131, 110702 (2020)

88. S. Babu, M. Krishnan, P. Rajagopal, V. Periyasamy, V. Veeraraghavan, R. Govindan, S. Jayaraman, Eur. J. Pharmacol. 873, 173004 (2020)

89. A. Fiorentino, B.D. Abrosca, S. Pacifico, C. Mastellone, M. Scognamiglio, P. Monaco, J. Agric. Food Chem. 57, 4148-4155 (2009)

90. M. Ahmad Khan, A.H.M.G. Sarwar, R. Rahat, R.S. Ahmed, S. Umar, Int. Immunopharmacol. 85, 106642 (2020)

91. H.W. Lim, J.G. Shim, H.K. Choi, M.W. Lee, Saengyak Hakhoechi. 36, 245-251 (2005)

92. D.S. Jang, G.Y. Lee, Y.M. Lee, Y.S. Kim, H. Sun, D.H. Kim, J.S. Kim, Chem. Pharm. Bull. 57, 397-400 (2009)

93. B. Riyana, D.H. Putri Huspa, M.H. Satari, D. Kurnia, Lett. Drug Des. Discov. 17, 1531-1537 (2020)

94. J. Chang, R. Case, Planta Med. 71, 955-959 (2005)

95. J. Michaud, M. Ane-Margail, Bull. Soc. Pharm. Bordeaux. 116, 52-64 (1977)

96. J. Wang, X. Fang, L. Ge, F. Cao, L. Zhao, Z. Wang, W. Xiao, PLoS ONE 13(5), e0197563 (2018)

97. S. Bakhshii, S. Khezri, R. Ahangari, A. Jahedsani, A. Salimi, Drug Dev. Res. (2021). https://doi.org/10.1002/ddr.21790

98. M.A. Arowosegbe, O.T. Amusan, S.A. Adeola, O.B. Adu, I.A. Akinola, B.F. Ogungbe, O.I. Omotuyi, G.M. Saibu, A.J. Ogunleye, R.I. Kanmodi, N.E. Lugbe, O.J. Ogunmola, D.C. Ajayi, S.O. Ogun, F.O. Oyende, A.O. Bello, P.G. Ishola, P.E. Obasieke, Curr. Drug Discov. Technol. 17, 682-695 (2020)

99. B.D. Sloley, L.J. Urichuk, P. Morley, J. Durkin, J.J. Shan, P.K.T. Pang, R.T. Coutts, J. Pharm. Pharmacol. 52, 451-459 (2000)

100. H.L. Xin, Y.C. Wu, Y.H. Su, J.Y. Sheng, C.Q. Ling, Planta Med. 77, 70-73 (2011)

101. X. Chang, B. Ma, L. He, Y. Xiao, X. Li, Chin. Tradit. Herb. Drugs. 24, 283-285 (1993)

102. J. Lu, Y. Jin, G. Liu, N. Zhu, M. Gui, A. Yu, X. Li, Chem. Nat. Compd. 46, 205-208 (2010)
103. M. Horiuch, C. Murakami, N. Fukamiya, D. Yu, K.H. Lee, J. Nat. Prod. 69, 1271-1274 (2006)

104. G. Eerduna, D. Wei, X. Yu, S. Qu, D. Sui, Pharmazie 68, 453$458(2013)$

105. G.N. He, B.C. Wang, H. Wang, L. Fan, X.M. Hu, Chin. Arch. Tradit. Chin. Med. 31, 2353-2355 (2016)

106. C. Liu, D. Weir, P. Busse, N. Yang, Z. Zhou, C. Emala, X.M. Li, Phytother. Res. 29, 925-932 (2015)

107. R.F. Webby, N. Z. J. Crop Hortic. Sci. 18, 1-4 (1990)

108. M. Xiang, C. Jin, R. Kou, G. Yang, J. Li, J. Huazhong, Norm. Univ. Nat. Sci. 49, 397-401 (2015)

109. J. Lu, G. Cui, X. Wang, N. Zhu, G. Liu, X. Li, Y. Jin, Chin. Pharm. J. 44, 328-330 (2009)

110. A.S. Syed, J.S. Jeon, C.Y. Kim, Nat. Prod. Res. 31, 1501-1508 (2017)

111. J. Lu, X.W. Li, M.Y. Gui, G.Y. Liu, N. Zhu, A.M. Yu, T. Okuyama, B. Masaki, Y.R. Jin, Chem. J. Chin. Univ. 30, 468-473 (2009)

112. R.F. Webby, K.R. Markham, Phytochemistry 29, 289-292 (1990)

113. Y. Jin, M. Gui, X. Li, CN1566127A.

114. A. Kalandiya, M. Vanidze, S. Papunidze, I. Chkhikvishvili, A. Shalashvili, Bull. Georgian Acad. Sci. 163, 157-159 (2001)

115. R.F. Webby, Phytochemistry 30, 2443-2444 (1991)

116. C.T. Luo, H.H. Zheng, S.S. Mao, M.X. Yang, C. Luo, H. Chen, Planta Med. 80, 201-208 (2014)

117. T.X. Shi, S. Wang, K.W. Zeng, P.F. Tu, Y. Jiang, Bioorg. Med. Chem. Lett. 23, 5904-5908 (2013)

118. W. Li, Y. Ding, T.H. Quang, T.T.N. Nguyen, Y.N. Sun, X.T. Yan, S.Y. Yang, C.W. Choi, E.J. Lee, K.Y. Paek, Y.H. Kim, Bull Korean Chem. Soc. 34, 1407-1413 (2013)

119. D.J. Comeskey, M. Montefiori, P.J.B. Edwards, T.K. McGhie, J. Agric. Food Chem. 57, 2035-2039 (2009)

120. D. Ferrari, F. Cimino, D. Fratantonio, M.S. Molonia, R. Bashllari, R. Busa, A. Saija, A. Speciale, Mediat. Inflamm. 2017, 3454023 (2017)

121. S.R. Pereira, L.M. Almeida, T.C.P. Dinis, J. Funct. Foods. 63 , $103586(2019)$

122. G.C. Di, R. Acquaviva, R. Santangelo, V. Sorrenti, L. Vanella, V.G. Li, N.D. Orazio, A. Vanella, F. Galvano, J. Evid. Based Complement. Altern. Med. 20, 285750 (2012)

123. X. Ma, S. Ning, Phytother. Res. 33, 81-89 (2019)

124. P. Zhang, S. Liu, Z. Zhao, L. You, M.D. Harrison, Z. Zhang, Food Chem. 343, 128482 (2021)

125. W. Fu, C. Tan, X. Meng, L. Lu, S. Jiang, D. Zhu, Chin. J. Med. Chem. 20, 116-118 (2010)

126. C.S. Sharanya, K.G. Arun, A. Sabu, M. Haridas, Prostaglandins Other Lipid Mediat. 150, 106453 (2020)

127. W. Ma, C. Liu, J. Li, M. Hao, Y. Ji, X. Zeng, Photochem. Photobiol. Sci. 19, 485-494 (2020)

128. S. Jangra, B. Sharma, S. Singh, Mater. Res Innov. 25, 264-275 (2020)

129. M.R. De Oliveira, I.C.C. De Souza, F.B. Brasil, Neurochem. Res. 46, 482-493 (2020)

130. S.W. Leung, J.H. Lai, J.C.C. Wu, Y.R. Tsai, Y.H. Chen, S.J. Kang, Y.H. Chiang, C.F. Chang, K.Y. Chen, Int. J. Mol. Sci. 21, $2899(2020)$

131. Q. Li, J. Gao, X. Pang, A. Chen, Y. Wang, Front. Pharmacol. 11, 559607 (2020)

132. C. Wang, L. Guo, J. Hao, L. Wang, W. Zhu, J. Nat. Prod. 79, 2977-2981 (2016)

133. Z. Ji, X. Liang, Acta Pharm Sin. 20, 778-781 (1985)

134. M.Y. Ali, S. Jannat, H.A. Jung, B.S. Min, P. Paudel, J.S. Choi, J. Food Biochem. 42, e12439 (2018)

135. H.W. Lim, S.J. Kang, M. Park, J.H. Yoon, B.H. Han, S.E. Choi, M.W. Lee, Nat. Prod. Sci. 12, 221-225 (2006) 
136. J.H. Ahn, Y. Park, Y.H. Jo, S.B. Kim, S.W. Yeon, J.G. Kim, A. Turk, J.Y. Song, Y. Kim, B.Y. Hwang, M.K. Lee, Food Chem. 308, 125666 (2020)

137. J. He, B.Z. Ma, X.X. Wang, F. Liu, W.J. Qin, X.I. Zhang, T. Zhao, Chin. Pharm. J. 50, 1960-1963 (2015)

138. D. Kwon, G.D. Kim, W. Kang, J.E. Park, S.H. Kim, E. Choe, J.I. Kim, J.H. Auh, J. Korean Soc. Appl. Biol. Chem. 57, 473-479 (2014)

139. X.H. Gao, S.D. Zhang, L.T. Wang, L. Yu, X.I. Zhao, H.Y. Ni, Y.Q. Wang, J.D. Wang, C.H. Shan, Y.J. Fu, Molecules 25, 1385 (2020)

140. J. Hu, X. Han, X. Li, B. Huang, Med. Plant. 9, 9-13 (2018)

141. A. Zeng, X. Liang, S. Zhu, C. Liu, S. Wang, Q. Zhang, J. Zhao, D.L. Song, Oncol. Rep. 45, 717-727 (2021)

142. D. Wang, L. Tian, H. Lv, Z. Pang, D. Li, Z. Yao, S. Wang, Biomed. Pharmacother. 132, 110773 (2020)

143. J.S. Lopez-Gonzalez, H. Prado-Garcia, D. Aguilar-Cazares, J.A. Molina-Guarneros, J. Morales-Fuentes, J.J. Mandoki, Lung Cancer 43, 275-283 (2004)

144. V.M. Navarro-Garcia, G. Rojas, M. Aviles, M. Fuentes, G. Zepeda, Mycoses 4, e569-e571 (2011)

145. J.R.S. Hoult, M. Paya, Gen. Pharmacol. 27, 713-722 (1996)

146. H. Li, Y. Yao, L. Li, J. Pharm. Pharmacol. 69, 1253-1264 (2017)

147. J.F. Vasconcelos, M.M. Teixeira, J.M. Barbosa-Filho, M.F. Agra, X.P. Nunes, A.M. Giulietti, R. Ribeiro-dos-Santos, M.B.P. Soares, Eur. J. Pharmacol. 609, 126-131 (2009)

148. R. Rashmi, N. Prakash, D. Rathnamma, S. Rao, A. Sahadev, C.R. Santhosh, U. Sunilchandra, K.S. Naveen, R.S. Wilfred, G.P. Kalmath, K.R.A. Kumar, H.M. Yathish, P. Waghe, Pharma Innov. 8, 29-35 (2019)

149. R. Rashmi, N. Prakash, D. Rathnamma, S. Rao, A. Sahadev, C.R. Santhosh, U. Sunilchandra, N.S. Kumar, W.S. Ruban, G.P. Kalmath, H. Dhanalakshmi, L. G, A.R. Gomes, K.R.A. Kumar, P. Waghe, Pharma Innov. 8, 36-40 (2019).

150. W.W. Fu, C.H. Tan, L.L. Lu, X.X. Meng, H.F. Luo, D.Y. Zhu, Chin. J. Nat. Med. 8, 247-249 (2010)

151. H.C. Chang, S.W. Wang, C.Y. Chen, T.L. Hwang, M.J. Cheng, P.J. Sung, K.W. Liao, J.J. Chen, Molecules 25, 5911 (2020)

152. A.M. Hirsch, A. Longeon, M. Guyot, Biochem. Syst. Ecol. 30, 55-60 (2002)

153. Y. Li, C. Ma, J. Huang, Chin. Pharm. J. 44, 1294-1297 (2009)

154. Y. Kimura, M. Sumiyoshi, Eur. J. Pharmacol. 746, 115-125 (2015)

155. B. Aouey, A.M. Samet, H. Fetoui, M.S.J. Simmonds, M. Bouaziz, Biomed. Pharmacother. 84, 1088-1098 (2016)

156. S. Ren, Y. Xing, C. Wang, F. Jiang, G. Liu, Z. Li, T. Jiang, Y. Zhu, D. Piao, Int. J. Biochem. Cell Biol. 125, 105777 (2020)

157. P. Wu, W. He, Y. Fu, J. Wu, J. Li, L. Xiao, Immunol. J. 36, 22-28 (2020)

158. B. Hwang, J. Lee, Q.H. Liu, E.R. Woo, D.G. Lee, Molecules 15, 3507-3516 (2010)

159. P.C. Kuo, H.Y. Hung, C.W. Nian, T.L. Hwang, J.C. Cheng, D.H. Kuo, E.J. Lee, S.H. Tai, T.S. Wu, J. Nat. Prod. 80, 1055-1064 (2017)

160. X.L. Ouyang, L.X. Wei, H.S. Wang, Y.M. Pan, S. Afr, J. Bot. 98, 162-166 (2015)

161. A. Wikul, T. Damsud, K. Kataoka, P. Phuwapraisirisan, Bioorg. Med. Chem. Lett. 22, 5215-5217 (2012)

162. Y. Zhang, H. Zhao, Y. Di, Q. Li, D. Shao, J. Shi, Q. Huang, J. Funct. Foods. 45, 206-214 (2018)
163. I. Paterniti, D. Impellizzeri, M. Cordaro, R. Siracusa, C. Bisignano, E. Gugliandolo, A. Carughi, E. Esposito, G. Mandalari, S. Cuzzocrea, Nutrients 9, 915 (2017)

164. A.N. Winter, M.C. Brenner, N. Punessen, M. Snodgrass, C. Byars, Y. Arora, D.A. Linseman, Oxid. Med. Cell. Longevity. 2017, 6297080 (2017)

165. Y.H. Wang, Y. Gao, Z. Li, D.I. Wang, W.H. Ling, Acta Nutr. Sin. 36, 53-57 (2014)

166. P. Thomas, E. Essien, A. Udoh, B. Archibong, O. Akpan, E. Etukudo, M. Leo, O. Eseyin, G. Flamini, K. Ajibesin, J. Ethnopharmacol. 269, 113737 (2021)

167. K.V. Wurms, J.M. Cooney, Asian J. Biochem. 1, 325-332 (2006)

168. X. Qin, C.H. Zhang, D.L. Yao, J.M. Cui, G. Li, J. Yanbian Med. Coll. 36, 187-189 (2013)

169. D.A. Sumilat, H. Yamazaki, K. Endo, H. Rotinsulu, D.S. Wewengkang, K. Ukai, M. Namikoshi, J. Nat. Med. 71, 776-779 (2017)

170. M.H. Farah, G. Samuelsson, Planta Med. 58, 14-18 (1992)

171. J. He, B.Z. Ma, T. Zhao, W. Wang, F.L. Wei, J. Lu, X.L. Zhang, Chin. Pharm. J. 49, 184-186 (2014)

172. Z.Q. Chang, E. Gebru, S.P. Lee, M.H. Rhee, J.C. Kim, H. Cheng, S.C. Park, J. Nutr. Sci. Vitaminol. 57, 118-122 (2011)

173. A. Fiorentino, C. Mastellone, B.D. Abrosca, S. Pacifico, M. Scognamiglio, G. Cefarelli, R. Caputo, P. Monaco, Food Chem. 115, 187-192 (2009)

174. T. Sakan, A. Fujino, F. Murai, Y. Butsugan, A. Suzui, Bull. Chem. Soc. Jpn. 32, 315-316 (1959)

175. D. Gross, W. Berg, H.R. Schuette, Phytochemistry 11, 30823083 (1972)

176. J. Ren, E.J. Han, S.H. Chung, Arch. Pharmacal. Res. 30, 708-714 (2007)

177. J. Kim, M. Ahn, Y. Choi, T. Kang, J. Kim, N.H. Lee, G.O. Kim, T. Shin, Inflammation 43, 1876-1883 (2020)

178. M. Vara-Messler, M.E. Pasqualini, A. Comba, R. Silva, C. Buccellati, A. Trenti, L. Trevisi, A.R. Eynard, A. Sala, C. Bolego, M.A. Valentich, Eur. J. Nutr. 56, 509-519 (2017)

179. Y. Mounika, R.M. Naik, Int. J. Pharm. Pharm. Res. 17, 306-328 (2019)

180. T. Suanarunsawat, G. Anantasomboon, C. Piewbang, Exp. Ther. Med. 11, 832-840 (2016)

181. P.U.M. Devi, P.S. Reddy, N.R.U. Rani, K.J. Reddy, M.N. Reddy, P. Reddanna, Eur. J. Plant Pathol. 106, 857-865 (2000)

182. M.H. Bang, I.G. Chae, E.J. Lee, N.I. Baek, Y.S. Baek, D.Y. Lee, I.S. Lee, S.P. Lee, S.A. Yang, Biosci. Biotechnol. Biochem. 76, 289-293 (2012)

183. K. Kono, A. Yamashita, T. Ishihara, JP2008120772A.

184. G.N. He, X.M. Hu, H. Wang, L. Fan, B.C. Wang, China J. Chin. Mater. Med. 30, 498-500 (2015)

185. Y. Lai, D.P. Xu, Chin. Tradit. Herb. Drugs. 30, 166-168 (2007)

186. P. Roger, J. P. Fournier, A. Martin, J. Choay, EP238401A2.

187. X. Chen, S. Yang, S. Bai, Chin. Tradit. Herb. Drugs. 42, 841-843 (2011)

188. J. Liang, H. Zhen, S. Li, W. Zhang, X. Wang, C. Liang, China J. Chin. Mater. Med. 33, 1275-1277 (2008)

189. X. Chang, B. Ma, J. Shan, L. Chen, Chin. Tradit. Herb. Drugs. 27, 395 (1996)

190. P. Li, A. Lu, B. Ma, J. Wei, China J. Chin. Mater. Med. 17, 420-421 (1992) 